\title{
Controle Fuzzy via Alocação de Pólos com Funções de Lyapunov por Partes
}

Eduardo Stockler Tognetti

Dissertação de Mestrado apresentada à Escola de Engenharia de São Carlos, da Universidade de São Paulo, como parte dos requisitos para obtenção do Título de Mestre em Engenharia Elétrica.

ORIENTADOR: Profa. Dra. Vilma Alves de Oliveira

São Carlos

2006 
Aos meus pais, Marcos e Vera, à minha esposa Taís, ao meu irmão Rodrigo, e a Deus acima de tudo. 


\section{Agradecimentos}

À minha orientadora Profa. Vilma Alves de Oliveira por ter me concedido a oportunidade de realizar este trabalho, pela orientação e pela amizade.

Ao Prof. Edson Gesualdo pelo companheirismo e por todo o apoio e incentivo.

À minha esposa Taís pela paciência e por estar ao meu lado em todos os momentos.

Aos meus pais por todo esforço que realizaram para que eu chegasse até aqui.

Aos docentes e funcionários do Departamento de Engenharia Elétrica da Escola de Engenharia de São Carlos, pela atenção recebida.

À todos os amigos, que nos felicitam na jornada.

Aos colegas do "LAC - Laboratório de controle".

À Votoratim Celulose e Papel pelas horas disponibilizadas, aos colegas de trabalho pelo companheirismo e aprendizado, em especial, ao Eng. ${ }^{\circ}$ Claudio Racca Jr. pela chefia e confiança em meu potencial.

À todos aqueles que direta ou indiretamente contribuíram para a realização deste trabalho. 


\section{Conteúdo}

Agradecimentos

Lista de Figuras iv

Resumo viii

$\begin{array}{lll}\text { Abstract } & \text { ix }\end{array}$

1 Introdução 1

1.1 Organização do Texto . . . . . . . . . . . . . . . . . . 4

2 Desigualdades Matriciais Lineares 5

2.1 Complemento de Schur . . . . . . . . . . . . . . . . . . . 7

2.2 Procedimento $\mathrm{S} \ldots \ldots \ldots \ldots$

2.3 Análise de Estabilidade e Síntese de Controladores via LMIs . . . 9

2.3.1 Estabilidade Exponencial . . . . . . . . . . . . . . . 9

2.3.2 Estabilidade Quadrática . . . . . . . . . . . . . 11

2.3.3 Síntese de Controladores . . . . . . . . . . . . . . . . 12

2.3.4 D-Estabilidade . . . . . . . . . . . . . . . . 14

$\begin{array}{llr}3 & \text { Sistemas Fuzzy } & 21\end{array}$

3.1 Modelagem Fuzzy Takagi-Sugeno . . . . . . . . . . . . . . . 21

3.1.1 Linearização de Dinâmicas Não-Lineares . . . . . . . . . . 23

3.1.2 Aproximação de Sistemas Não-Lineares por Sistemas Lineares Fuzzy T-S . . . . . . . . . . . . . . . 30

3.2 Controladores Fuzzy Takagi-Sugeno . . . . . . . . . . . . . . . 40 
3.2.1 Compensação Distribuída Paralela . . . . . . . . . . . . . 40

3.2 .2 Condições de Estabilidade . . . . . . . . . . . . . . . . . 41

3.2.3 Projeto de Controladores Estáveis . . . . . . . . . . . . . 42

4 Controle Linear por Partes $\quad 51$

4.1 Introdução . . . . . . . . . . . . . . . . . . . . . 51

4.2 Modelagem Linear por Partes . . . . . . . . . . . . . . . . . . . . 52

4.3 Estabilidade de Sistemas Lineares por Partes . . . . . . . . . . 54

4.4 Projeto de Controladores . . . . . . . . . . . . . . . . . . 59

4.5 Sistemas Fuzzy com Funções de Lyapunov por Partes . . . . . . . 61

4.5.1 Estabilidade e Síntese de Controladores . . . . . . . . . . . 61

5 Sistemas Fuzzy com Restrição de Alocação de Pólos $\quad 75$

5.1 D-Estabilidade com Funções de Lyapunov Quadráticas . . . . . . 77

$5.2 \mathcal{D}$-Estabilidade com Funções de Lyapunov por Partes . . . . . . . 81

5.3 Resultados de Simulação . . . . . . . . . . . . . . . . . 86

5.3.1 Projeto do controlador . . . . . . . . . . . . . . . 87

6 Conclusão e Proposta para Trabalhos Futuros 101

6.1 Conclusão . . . . . . . . . . . . . . . . . . . . . . . 101

6.2 Proposta para Trabalhos Futuros . . . . . . . . . . . . . 102 


\section{Lista de Figuras}

2.1 Combinação convexa dos pontos $x, y$ com $0 \leq \alpha \leq 1$. . . . . . . 7

2.2 Regiões LMIs - (a) semi-plano, (b) disco e (c) setor cônico. . . . . 16

2.3 Região circular para alocação de pólos. . . . . . . . . . . . . . . 17

2.4 Resposta do sistema em malha fechada para a condição inicial $x_{0}^{T}=[1.5-1]$. A linha contínua corresponde ao controlador obtido pela resolução da LMI (2.19) e a linha tracejada ao controlador

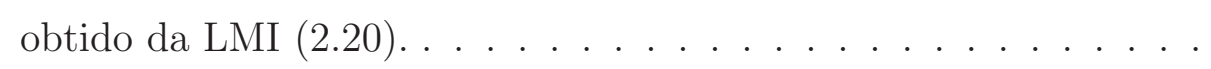

3.1 Trajetória do modelo global composta por dois modelos locais. Os modelos locais (a) e (b) são distintos, porém, resultam na mesma trajetória. . . . . . . . . . . . . . . . 27

3.2 Campo vetorial do sistema $\sum_{1}$. . . . . . . . . . . . . . . . 29

3.3 Campo vetorial do sistema $\sum_{2}$. . . . . . . . . . . . 30

3.4 Campo vetorial de $\sum_{1}$ na região $R$. . . . . . . . . . . . . . 30

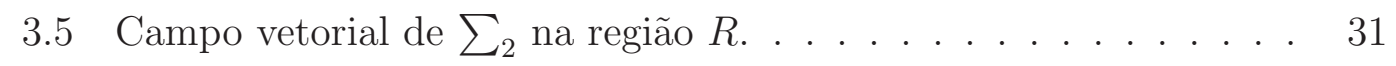

3.6 Função não-linear $f\left(x_{1}, x_{2}\right)=8 x_{1}+10 x_{2} \operatorname{sen}\left(4 x_{1}\right)+x_{1}^{3}-4 x_{1} x_{2} . \quad$. 32

3.7 Modelo fuzzy T-S construído. . . . . . . . . . . . . . . . 32

3.8 Erro de aproximação da função não-linear pelo modelo fuzzy T-S. 33

3.9 Modelo não-linear e modelos locais. . . . . . . . . . . . . . 36

3.10 Funções de pertinência fuzzy. . . . . . . . . . . . . . . . . . . 37

3.11 Modelo global fuzzy utilizando ambos os modelos lineares. . . . . 37

3.12 Modelo global fuzzy utilizando modelo local afim. . . . . . . . . . 38

3.13 Funções pertinências dos 11 modelos lineares locais. . . . . . . . . 38

3.14 Modelo global fuzzy com o uso de 11 modelos lineares locais. . . . 39 
3.15 Função de pertinência adequada. . . . . . . . . . . . . . . . . . 39

3.16 Modelo global fuzzy com a função pertinência modificada. . . . . 40

3.17 Carro com um pêndulo invertido. . . . . . . . . . . . . . . . 44

3.18 Funções de pertinência fuzzy. . . . . . . . . . . . . . . . . . . . . 47

3.19 Modelo do pêndulo invertido no Simulink. . . . . . . . . . . . . . 48

3.20 Sistema em malha fechada com o controlador fuzzy. . . . . . . . . 48

3.21 Estrutura do controlador fuzzy via CDP. . . . . . . . . . . . 49

3.22 Controlador linear. . . . . . . . . . . . . . . . . . . . . . . . 49

3.23 Resposta do ângulo do pêndulo para diversas condições iniciais com o controlador fuzzy CDP (linha contínua) e o controlador linear clássico (linha tracejada). . . . . . . . . . . . . . 50

4.1 Uma realimentação linear saturada (esquerda) leva a um sistema linear por partes com uma divisão poliedral do espaço de estado (direita). . . . . . . . . . . . . . . . 53

4.2 Trajetórias do sistema. Os pontos em preto significam a condição inicial da trajetória. . . . . . . . . . . . . . . . 56

4.3 (a) Funções de pertinência. (b) Divisão no espaço de estado em partições.

4.4 Funções de pertinência fuzzy das variáveis linguísticas "Muito Negativo", "Negativo", "Positivo"e "Muito Positivo"representadas pelas variáveis $\mu_{1}, \mu_{2}, \mu_{3}$ e $\mu_{4}$ respectivamente. . . . . . . . 68

4.5 Partições no espaço de estados. . . . . . . . . . . . . . . . . 70

4.6 Sistema em malha fechada. . . . . . . . . . . . . . . . . . . 72

4.7 Modelo fuzzy T-S. . . . . . . . . . . . . . . . . . . . . 72

4.8 Matriz de estado $A(\mu)$ ponderada. . . . . . . . . . . 73

4.9 Controlador chaveado. . . . . . . . . . . . . . . . 73

4.10 Resposta do estado $\left[\begin{array}{llll}x_{1} & x_{2} & x_{3} & x_{4}\end{array}\right]^{T}$ à condição inicial $x_{0}=\left[\begin{array}{llll}4 & -10 & 0 & 0\end{array}\right] .74$

5.1 Sistema com uma bola em equilíbrio em uma trave. . . . . . . . . 88

5.2 Funções de pertinência fuzzy. . . . . . . . . . . . . . . . . . . . . 90

5.3 Diagrama Simulink do sistema não-linear em malha aberta. . . . . 95 
5.4 Diagrama Simulink do sistema Bola-Trave. . . . . . . . . . . . . . 95

5.5 Diagrama Simulink do sistema em malha fechada com o controlador fuzzy via CDP. . . . . . . . . . . . . . . . . . . 95

5.6 Diagrama Simulink da estrutura interna do controlador fuzzy via

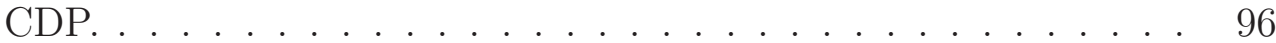

5.7 Diagrama Simulink do sistema em malha fechada com o controlador chaveado fuzzy. . . . . . . . . . . . . . . . . . . 96

5.8 Estrutura do controlador chaveado fuzzy. . . . . . . . . . . . . . . 96

5.9 Pólos de malha fechada dos subsistemas lineares do caso 1. . . . . 97

5.10 Pólos de malha fechada dos subsistemas lineares locais do caso 2.97

5.11 Localização dos pólos dos subsistemas de malha fechada do caso 3.98

5.12 Resposta do sistema em malha fechada para os casos 1, 2 e 3 com condição inicial $\left[\begin{array}{llll}1 & 0 & 0 & 0\end{array}\right] \ldots \ldots$. . . . . . . . . . . . . . . . . . 99

5.13 Resposta do sinal de controle para os casos 1, 2 e 3 com condição inicial $\left[\begin{array}{llll}1 & 0 & 0 & 0\end{array}\right] \ldots \ldots \ldots \ldots$ 


\section{Resumo}

Tognetti, Eduardo Stockler (2006).Controle Fuzzy via Alocação de Pólos com Funções de Lyapunov por Partes. Dissertação (Mestrado), Escola de Engenharia de São Carlos, Universidade de São Paulo, São Carlos, 2006.

O presente trabalho apresenta um método de projeto de controlador com alocação de pólos em sistemas fuzzy utilizando funções de Lyapunov por partes e contínuas no espaço de estado. A idéia principal é utilizar controladores chaveados no espaço de estado para obter uma resposta transitória satisfatória do sistema, obtida pela localização dos pólos. A modelagem fuzzy T-S é utilizada para representar um sistema não-linear em diversos pontos de linearização através de uma aproximação por vários modelos locais lineares invariantes no tempo. A análise de estabilidade e o projeto de sistemas de controle podem se formulados em termos de Desigualdades Matriciais Lineares (em inglês, Linear Matrix Inequalities (LMIs)), as quais são resolvidas por técnicas de programação convexa. Na análise de estabilidade ou na síntese de um controlador em sistemas fuzzy é necessário resolver um número determinado de LMIs de acordo com o número de modelos locais. Encontrar uma função de Lyapunov comum a todos os modelos locais pode ser inviável, especialmente quando se impõem critérios de desempenho, que aparecem como restrições no contexto de LMIs. A proposta de uma função de Lyapunov por partes objetiva diminuir o conservadorismo na busca de um controlador que leve os pólos de malha fechada à uma região desejada. Resultados de análise e síntese da teoria de sistemas lineares por partes contribuíram para a construção do resultado apresentado. Exemplos com simulação ilustram o método proposto.

Palavras-Chave: Modelagem Fuzzy T-S, LMIs, Alocação de Pólos, Sistema Linear por Partes, Função de Lyapunov por Partes. 


\section{Abstract}

Tognetti, Eduardo Stockler (2006).Fuzzy Pole Placement Based on Piecewise Lyapunov Functions. Dissertation (Master), Escola de Engenharia de São Carlos, Universidade de São Paulo, São Carlos, 2006.

This work presents a controller design method for fuzzy dynamic systems based on piecewise Lyapunov functions with constraints on the closed-loop pole location. The main idea is to use switched controllers to locate the poles of the system to obtain a satisfactory transient response. The pole placement strategy allows to specify the performance in terms of the desired time response of the feedback system. The Takagi-Sugeno fuzzy model can approximate the nonlinear system in several linearization points using linear time invariant systems. Thus, a global fuzzy model can be obtained from a fuzzy combination of these linear systems. Stability analysis and design of fuzzy control systems can be efficiently carried out in the context of linear matrix inequalities (LMIs). If the fuzzy system is described by many local models, the resulting set of LMIs may be infeasible. The search for a Lyapunov function in the fuzzy pole placement problem may be easier to be satisfied in a piecewise framework. Some results from piecewise linear systems theory have contributed to the development of the presented technique. Some examples are given to illustrate the proposed method.

Key-words: Fuzzy Systems, Linear Matrix Inequalities (LMIs), Pole Placement, Piecewise Linear Systems, Piecewise Lyapunov Function. 


\section{Capítulo 1}

\section{Introdução}

$\mathrm{Na}$ teoria de controle muitas técnicas de análise de estabilidade e projeto de sistemas atendendo condições de desempenho e robustez têm sido desenvolvidas. Para a análise de um sistema real, a primeira etapa é a sua representação matemática. A construção de modelos matemáticos envolve uma teoria extensa que busca garantir uma melhor aproximação do sistema real para representar as suas principais características dinâmicas.

O estudo de sistemas dinâmicos freqüentemente envolve a linearização em pontos de interesse para uma análise mais precisa ou para o projeto de uma estratégia de controle eficiente nesta região.

A linearização em um ponto de operação fornece o comportamento da dinâmica na vizinhança deste ponto. Nem sempre, porém, a dinâmica se limita a esta vizinhança e em pontos afastados o comportamento pode ser muito diferente do modelo linear obtido no ponto de operação em que o sistema foi linearizado. Ocorrendo isto, os requisitos de projeto podem não ser mais atendidos, pois o controlador projetado não irá mais satisfazer as condições de desempenho e o sistema pode até se tornar instável.

Os modelos fuzzy Takagi-Sugeno (T-S) permitem representar a dinâmica nãolinear em vários pontos de operação ou linearização (Takagi e Sugeno, 1985). A idéia destes modelos consiste na descrição aproximada de um sistema nãolinear como a combinação de sistemas lineares locais invariantes no tempo, que descrevem o comportamento aproximado em diversos pontos no espaço de estado. 
Desta forma, a linearização em um único ponto é um caso particular de modelo fuzzy T-S com apenas um modelo local.

O modelo global é obtido através da combinação fuzzy dos modelos lineares locais. A idéia é que os requisitos de projetos dos modelos locais possam valer para o modelo global. Para isto, em geral, um controlador linear de realimentação é projetado para cada modelo local. O controlador global resultante, o qual é nãolinear em geral, é uma combinação fuzzy de cada controlador linear individual.

A análise de estabilidade representa um ponto muito importante no estudo de sistemas dinâmicos. Mas, no problema de controle é desejável que vários requisitos sejam atendidos. A transformação em um problema convexo é uma formulação que possibilita atender a um conjunto de exigências. A principal vantagem da formulação LMI (do inglês, Linear Matrix Inequalities) é a sua versatilidade em combinar várias restrições ou objetivos de projeto em uma maneira numericamente tratável (Boyd et al., 1994). Existem diversos aplicativos que resolvem eficientemente LMIs numericamente (Gahinet et al., 1995).

Em sistemas fuzzy T-S, a análise de estabilidade e síntese geralmente requerem que um número elevado de LMIs sejam satisfeitas, proporcionais ao número de modelos locais. O critério de estabilidade de Lyapunov para sistemas lineares é também utilizado para sistemas fuzzy através do uso da desigualdade de Lyapunov. Encontrar uma função de Lyapunov comum a todos os modelos locais que satisfaça condições de desempenho e estabilidade pode ser conservador para um número elevado de LMIs, além de apresentar, em geral, pouca factibilidade. Portanto, o uso de funções de Lyapunov por partes objetiva diminuir o conservadorismo na busca de soluções de LMIs e esta é a principal motivação do uso destas funções por partes neste trabalho.

Um dos principais trabalhos divulgados nesta área é o de Johansson e Rantzer (1998) para sistemas híbridos e Rantzer e Johansson (2000) para sistemas lineares, resultados da tese de Johansson (1999). Nessa tese, o autor trata da modelagem de sistemas dinâmicos usando sistemas lineares por partes. A idéia é dividir o espaço de estados de forma que cada partição seja governada por uma dinâmica. 
A principal contribuição dada foi na análise de estabilidade através do uso de funções de Lyapunov por partes. Porém, Rantzer e Johansson (2000) limitaramse ao problema de análise de sistemas afins pois sua formulação para síntese de controladores resultava em desigualdades matriciais bilineares (do inglês Bilinear Matrix Inequalities (BMI)). Posteriormente, outros trabalhos surgiram resolvendo o problema da síntese, como em Feng et al. (2002), com o projeto de controle $H_{\infty}$.

No próprio trabalho de Johansson (1999), os resultados são estendidos para a sua aplicação em sistemas fuzzy. Nesse trabalho, aborda-se a análise de estabilidade de sistemas fuzzy T-S afins com funções de Lyapunov contínuas por partes nas partições do espaço de estado. Através do Procedimento-S (Boyd et al., 1994) condições menos conservadoras de análise são obtidas porém o problema de síntese não é resolvido. Para o problema de síntese com funções de Lyapunov contínuas por partes em sistemas fuzzy afins, Feng e Sun (2002) utilizaram um controlador $H_{2}$. Em Feng (2003), o autor aborda a síntese de controladores $H_{\infty}$ para sistemas lineares fuzzy com funções de Lyapunov por partes e contínuas nas partições do espaço de estado e em Feng et al. (2005) é utilizado BMIs para sistemas afins. Estes resultados motivaram o desenvolvimento de um projeto de controlador por partes para sistemas fuzzy T-S conjugado a uma condição de desempenho.

Um dos requisitos de desempenho a ser considerado é a resposta transitória. Em sistemas de controle a lentidão ou a oscilação de uma resposta pode comprometer a estabilidade ou impactar em custos. O comportamento da resposta temporal a uma entrada está diretamente relacionado ao posicionamento dos pólos do sistema. Assim, se o projetista escolher adequadamente a localização dos pólos de malha fechada, o sistema de controle irá atuar como desejado.

A localização precisa dos pólos de malha fechada pode ser obtida se o sistema for controlável. Entretanto, pode ser vantajoso definir uma região, ao invés de uma localização especifica, a qual os pólos devem pertencer. Uma das razões é que em sistemas incertos a localização dos pólos de malha fechada muda de acordo com a variação dos parâmetros incertos. 
A restrição de localização regional dos pólos pode ser obtida através de uma modificação na equação de Lyapunov (Haddad e Bernstein, 1992) ou na desigualdade de Lyapunov por meio de LMIs (Chilali et al., 1999). A estabilização robusta de sistemas incertos com restrição na alocação dos pólos tem aplicações, por exemplo, em sistemas de potência (Rao e Sen, 2000).

Em sistemas fuzzy, o problema de alocação de pólos também tem ganhado destaque. Em Joh et al. (1997) a restrição de alocação dos pólos de malha fechada é combinada com o resultado de síntese de Tanaka e Sugeno (1992). A técnica $H_{\infty}$ também é combinada para alcançar atenuação de distúrbios (Han et al., (2000); Wang et al., (2003); Assawinchaichote e Nguang, (2004)).

O presente trabalho tem o objetivo de desenvolver e contribuir para o projeto com alocação de pólos de sistemas fuzzy via funções de Lyapunov por partes e contínuas nas partições do espaço de estado com condições menos conservadores que as técnicas atuais.

\subsection{Organização do Texto}

Este texto está organizado na seguinte forma. O Capítulo 2 apresenta os principais resultados existentes na literatura de estabilidade e síntese via LMIs e suas principais propriedades na manipulação de desigualdades matriciais. Também introduz o conceito de $\mathcal{D}$-estabilidade e o projeto de alocação regional de pólos via LMIs. O Capítulo 3 apresenta a modelagem fuzzy Takagi-Sugeno, as condições de estabilidade e projeto de controladores utilizando a chamada compensação distríbuida paralela (CDP) (Wang et al., 1995). No Capítulo 4 é introduzido o controle linear por partes, a modelagem de plantas em sistemas por partes, análise de estabilidade e o projeto de controladores por partes no espaço de estado. A aplicação em sistemas fuzzy é abordada em seguida. No Capítulo 5 são utilizados os conceitos apresentados nos capítulos anteriores para introduzir a $\mathcal{D}$ estabilidade em sistemas fuzzy via funções de Lyapunov quadráticas e quadráticas por partes no espaço de estado. No Capítulo 6 são apresentadas as conclusões e proposta de trabalhos futuros. 


\section{Capítulo 2}

\section{Desigualdades Matriciais}

\section{Lineares}

Uma desigualdade matricial linear (LMI, do inglês linear matricial inequality) é uma desigualdade do tipo $F(x)>0$ com $F(x): \mathbb{R}^{m} \rightarrow \mathbb{R}^{n \times n}$ simétrica positivadefinida e afim nas variáveis de busca que são representadas pelo vetor $x$. Assim, uma LMI pode ser representada genericamente pela forma

$$
F(x)=F_{0}+\sum_{i=1}^{m} x_{i} F_{i}>0, \quad x=\left[\begin{array}{c}
x_{1} \\
\vdots \\
x_{m}
\end{array}\right],
$$

onde $F_{i}=F_{i}^{T} \in \mathbb{R}^{n \times n}, i=0, \cdots, m$ são matrizes dadas e $x_{i}, i=1, \cdots, m$ são variáveis escalares a serem determinadas de forma a satisfazer a desigualdade. Quando existe uma solução $x$ para $F(x)>0$ diz-se que a LMI é factível. A LMI (2.1) é equivalente ao conjunto de $n$ desigualdades polinomiais.

Uma LMI pode ser representada de várias formas e raramente aparece num problema na forma genérica afim (2.1). A vantagem de (2.1) é que toda LMI pode ser reescrita nesta forma e por isso todos os algoritmos de resolução de LMIs são desenvolvidos para esta representação.

Exemplo 2.1 Considere as desigualdades $P>0, A^{T} P+P A<0$. Para verificar que estas são LMIs basta notar que as funções $F(P)=A^{T} P+P A$ e $G(P)=P$ são afins em $P$ e portanto podem ser reescritas como em (2.1). Para um sistema 
de dimensão 2 tem-se:

$$
\begin{gathered}
P=\left[\begin{array}{ll}
p_{1} & p_{2} \\
p_{2} & p_{3}
\end{array}\right]=p_{1} G_{1}+p_{2} G_{2}+p_{3} G_{3}=G_{0}+\sum_{i=1}^{3} g_{i} G_{i}, \\
g=\left[\begin{array}{l}
p_{1} \\
p_{2} \\
p_{3}
\end{array}\right], G_{1}=\left[\begin{array}{ll}
1 & 0 \\
0 & 0
\end{array}\right], G_{2}=\left[\begin{array}{ll}
0 & 1 \\
1 & 0
\end{array}\right], G_{3}=\left[\begin{array}{ll}
0 & 0 \\
0 & 1
\end{array}\right], G_{0}=\left[\begin{array}{ll}
0 & 0 \\
0 & 0
\end{array}\right], \\
A^{T} P+P A=F_{0}+\sum_{i=1}^{3} g_{i} F_{i}, \\
F_{0}=0, \quad F_{1}=G_{1} A+A^{T} G_{1}, \quad F_{2}=G_{2} A+A^{T} G_{2}, \quad F_{3}=G_{3} A+A^{T} G_{3} .
\end{gathered}
$$

Assim, a LMI P $>0$ pode ser representada como $G(g)=G_{0}+\sum_{i=1}^{3} g_{i} G_{i}>0$ e a LMI $A^{T} P+P A<0$ como $F(g)=F_{0}+\sum_{i=1}^{3} g_{i} F_{i}<0$.

A LMI (2.1) é uma restrição convexa, isto é, o conjunto de todas as soluções possíveis de (2.1), $S=\{x: F(x)>0\}$, é um conjunto convexo. Para verificar isto, suponha que $x, y$ são duas soluções quaisquer da LMI, isto é, $F(x)>0$ e $F(y)>0$, então para qualquer combinação convexa de $x$ e $y$, representada por $\alpha x+(1-\alpha) y$, sendo $0 \leq \alpha \leq 1$ o coeficiente de combinação convexa, tem-se, devido a linearidade, $F(\alpha x+(1-\alpha) y)=\alpha F(x)+(1-\alpha) F(y)>0$ e portanto $\alpha x+(1-\alpha) y$ também é uma solução da LMI. Logo $S$ é convexo. Esta propriedade é importante do ponto de vista numérico pois tem-se garantia que o problema de encontrar uma solução qualquer de uma LMI consiste na busca de um elemento qualquer num conjunto convexo, como mostra a Figura 2.1, problema que pode ser resolvido de forma eficiente, com convergência global e tempo polinomial.

Vários problemas de restrição convexa podem ser escritos como LMI. Em particular, desigualdades lineares, desigualdades quadráticas (convexas), desigualdades de norma de matrizes, e restrições que aparecem na teoria de controle, como na teoria de Lyapunov, podem ser reescritas na forma de LMIs.

A solução de LMIs simultâneas é equivalente à solução de uma única LMI bloco-diagonal de dimensão maior. Por exemplo, as duas LMIs $F(x)<0$ e $G(x)>0$ podem, ser reescritas na forma $\operatorname{diag}\{-F(x), G(x)\}>0$ onde $\operatorname{diag}\{$. denota uma matriz bloco-diagonal. 


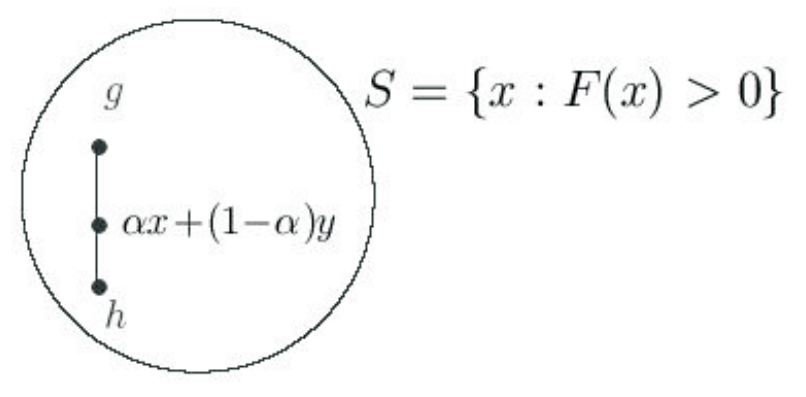

Figura 2.1: Combinação convexa dos pontos $x, y$ com $0 \leq \alpha \leq 1$.

Exemplo 2.2 (Propriedade de LMIs) Considere as LMIs $P>0$ e $A^{T} P+$ $P A<0$. Pode-se verificar que estas LMIs podem ser reescritas como

$$
\left[\begin{array}{cc}
-A^{T} P-P A & 0 \\
0 & P
\end{array}\right]>0 .
$$

Esta propriedade permite a incorporação de novas restrições ao problema original desde que essas novas restrições se apresentem como novas LMIs a serem satisfeitas. Maiores detalhes sobre esta e outras propriedades podem ser encontradas em Boyd et al. (1994).

\subsection{Complemento de Schur}

Grande parte dos problemas não aparece originalmente em forma de LMIs. Algumas ferramentas são bastante úteis na tarefa de reescrever o problema original através de LMIs. O complemento de Schur é uma ferramenta básica na manipulação de desigualdades matriciais.

Lema 2.1 (Boyd et al., 1994) Seja $x \in \mathbb{R}$ o vetor de variáveis de decisão e $M_{1}(x), M_{2}(x), M_{3}(x)$ funções afins de $x$ com $M_{1}(x), M_{2}(x)$ simétricas. Então, as seguintes afirmações são equivalentes

$$
\begin{aligned}
& \text { a) } M_{1}(x)-M_{3}(x)^{T} M_{2}(x)^{-1} M_{3}(x)>0 \operatorname{com} M_{2}(x)>0, \\
& \text { b) }\left[\begin{array}{cc}
M_{1}(x) & M_{3}(x)^{T} \\
M_{3}(x) & M_{2}(x)
\end{array}\right]>0 .
\end{aligned}
$$


Note que a) não é uma LMI, pois, $M(x)=M_{1}(x)-M_{3}(x)^{T} M_{2}(x)^{-1} M_{3}(x)$ não é uma função afim em $x$. No entanto, a desigualdade em a) é equivalente à desigualdade em b), que é uma LMI. Note que para satisfazer a), b) deve ter $M_{1}(x)>0$ e $M_{2}(x)>0$ como condições necessárias, porém não suficientes.

Exemplo 2.3 (Equivalência de LMIs) Sejam A, B, C, R matrizes dadas. Note que

$$
A^{T} P+P A-P B R^{-1} B^{T} P+C^{T} C<0 \operatorname{com} P>0, R>0
$$

não é uma LMI na variável $P$, pois é quadrática em P. Não é possível, aplicar o complemento de Schur para eliminar o termo quadrático, devido ao sinal deste termo.

Defina agora $S=P^{-1}$, o que implica que $S>0$. Pré e pós multiplicando (2.2) por $S$ tem-se

$$
S A^{T}+A S-B R^{-1} B^{T}+S C^{T} C S<0
$$

Agora (2.3) é quadrática em $S$ e o sinal do termo não linear é coerente com o complemento de Schur, que ao ser aplicado fornece a desigualdade

$$
\left[\begin{array}{cc}
-S A^{T}-A S+B R^{-1} B^{T} & S C^{T} \\
C S & -I
\end{array}\right]<0 \operatorname{com} S>0
$$

a qual é uma LMI em $S$ e que ao ser resolvida fornece $P$, pois $P=S^{-1}$ e satisfaz (2.2).

\subsection{Procedimento $\mathrm{S}$}

O procedimento S permite concatenar várias restrições escalares de desigualdade em uma única. Para reduzir o conservadorismo, este procedimento introduz multiplicadores como fatores de ponderação a serem determinados.

Sejam $T_{0}, \ldots, T_{p} \in \mathbb{R}^{n \times n}$ matrizes simétricas dadas. Considere a seguinte condição em $T_{0}, \ldots, T_{p}$ :

$$
\xi^{T} T_{0} \xi>0, \forall \xi \neq 0: \xi^{T} T_{i} \xi \geq 0, i=1, \ldots, p .
$$


Existem escalares $\tau_{i} \geq 0, i=1, \ldots, p$ tais que

$$
T_{0}-\sum_{i=1}^{p} \tau_{i} T_{i}>0
$$

então (2.5) é satisfeita. Porém, não é trivial mostrar que (2.5) e (2.6) são equivalentes para $p=1$. Note que (2.6) é uma LMI nas variáveis $T_{0}$ e $\tau_{1}, \ldots, \tau_{p}$. Maiores detalhes sobre o procedimento S podem ser encontrados em Boyd et al. (1994).

Exemplo 2.4 (Uso do procedimento S) Considere a seguinte restrição na variável P: para todo $\xi \neq 0$ e $\pi$ satisfazendo $\pi^{T} \pi \leq \xi^{T} C^{T} C \xi$,

$$
\left[\begin{array}{l}
\xi \\
\pi
\end{array}\right]^{T}\left[\begin{array}{cc}
A^{T} P+P A & P B \\
B^{T} P & 0
\end{array}\right]\left[\begin{array}{l}
\xi \\
\pi
\end{array}\right]<0 .
$$

Aplicando o procedimento $S$, (2.7) é equivalente à existência de $\tau \geq 0$ tal que

$$
\left[\begin{array}{cc}
A^{T} P+P A+\tau C^{T} C & P B \\
B^{T} P & -\tau I
\end{array}\right]<0 .
$$

Então, o problema de obter $P>0$ tal que (2.7) seja satisfeita pode ser expresso como uma LMI nas variáveis $P$ e $\tau$.

\subsection{Análise de Estabilidade e Síntese de Con- troladores via LMIs}

\subsubsection{Estabilidade Exponencial}

Como já discutido, a estabilidade tem um papel central na análise de sistemas dinâmicos. A estabilidade de um sistema pode ser definida de várias formas dependendo de como este se apresenta. Neste trabalho, a estabilidade será definida no senso de Lyapunov, ou seja, a partir da análise do comportamento das trajetórias de um sistema quando o estado inicial está próximo ao equilíbrio. Posteriormente, considera-se o caso de modelos distanciados do equilíbrio e como a análise de estabilidade se estende a estes casos. 
A estabilidade assintótica garante que o sistema tende ao repouso a partir de uma condição inicial suficientemente próxima ao ponto de equilíbrio. A estabilidade exponencial será de maior interesse no decorrer do trabalho visto que garante que a convergência do sistema para o equilíbrio será limitada por uma função exponencial no tempo. A taxa de decaimento das trajetórias para a origem (qualquer ponto de equilíbrio pode ser transladado para a origem) é de grande importância pois, num sistema linear, pode ser obtida através do posicionamento dos pólos de um sistema. Há certamente muitos sistemas assintoticamente estáveis cuja convergência não é exponencial. Para sistemas lineares, os conceitos de estabilidade assintótica e exponencial são coincidentes.

Definição 2.2 O ponto de equilíbrio $x=0$ é exponencialmente estável se existir constantes $r, a, b>0$ tal que

$$
\|x(t)\| \leq a\left\|x_{0}\right\| e^{-b\left(t-t_{0}\right)}, \quad \forall t \geq t_{0} \geq 0, \quad \forall\left\|x\left(t_{0}\right)\right\|<r
$$

O método direto de Lyapunov para sistemas dinâmicos introduz o conceito de energia de um sistema, indicado pela chamada função de Lyapunov $V(x)$. O método direto de Lyapunov segue abaixo.

Teorema 2.3 (Método Direto de Lyapunov) (Khalil, 1996) Seja $x=0$ um ponto de equilíbrio de $\dot{x}=f(x)$ e $\mathcal{D} \subset \mathbb{R}^{n}$ seja um domínio contendo $x=0$. Seja $V: \mathcal{D} \rightarrow \mathbb{R}$ uma função continuamente diferenciável, tal que

$$
\begin{gathered}
V(0)=0 \text { e } V(x)>0 \text { em } \mathcal{D}-\{0\} \\
\dot{V}(x) \leq 0 \text { em } \mathcal{D} .
\end{gathered}
$$

Então, $x=0$ é estável. Além disso, se

$$
\dot{V}(x)<0 \text { em } \mathcal{D}-\{0\}
$$

então $x=0$ é assintoticamente estável.

Prova: Vide Khalil (1996). 
A estabilidade de um ponto de equilíbrio de um sistema não-linear também pode ser investigada através da análise de estabilidade do sistema linearizado em torno deste ponto. Um ponto de equilíbrio do sistema não-linear é localmente exponencialmente estável se, e somente se, sua linearização em torno deste ponto for exponencialmente estável.

Teorema 2.4 (Método Indireto de Lyapunov) (Khalil, 1996) Seja $x=0$ o ponto de equilíbrio do sistema não-linear

$$
\dot{x}=F(x)
$$

no qual $F: \mathcal{D} \rightarrow \mathbb{R}^{n}$ é continuamente diferenciável e $\mathcal{D}$ uma vizinhança da origem. Seja

$$
A=\left.\frac{\partial F}{\partial x}(x)\right|_{x=0} .
$$

Então:

1. A origem é assintoticamente estável se todos os autovalores de A possuirem parte real negativa.

2. A origem é estável se um ou mais autovalores de A possuirem parte real positiva.

Prova: Vide Khalil (1996).

\subsubsection{Estabilidade Quadrática}

Por estabilidade quadrática entende-se a estabilidade que pode ser estabelecida por uma função de Lyapunov quadrática. Lyapunov estabeleceu que a existência de uma função de Lyapunov quadrática, dada por $V(x)=x^{T} P x, P>0$, é condição necessária e suficiente para a estabilidade assintótica de um sistema linear. Se existir tal função $P$, diz-se que o sistema linear é quadraticamente estável e $V$ é uma função de Lyapunov quadrática.

Considerando a função candidata de Lyapunov

$$
V(x)=x^{T} P x,
$$


onde $P$ é uma matriz real definida positiva . A derivada de $V$ ao longo das trajetórias de um sistema linear

$$
\dot{x}(t)=A x(t)
$$

é dado por

$$
\dot{V}(x)=x^{T} P \dot{x}+\dot{x}^{T} P x=x^{T}\left(P A+A^{T} P\right) x=-x^{T} Q x,
$$

onde $Q$ é uma matriz simétrica definida por

$$
P A+A^{T} P=-Q
$$

Se $Q$ for definida positiva, então pode-se concluir que a origem é assintoticamente estável, isto é, os autovalores de $A$ tem parte real negativa.

Pode-se utilizar também a desigualdade de Lyapunov, que leva a um resultado equivalente ao demonstrado acima (Boyd et al., 1994).

Teorema 2.5 A origem do sistema (2.8) é exponencialmente estável se existir uma matriz P simétrica positiva definida que satisfaça a seguinte desigualdade

$$
A^{T} P+P A<0
$$

A desigualdade (2.10) é referenciada como desigualdade de Lyapunov na qual $P$ é a variável e $A \in \mathbb{R}^{n \times n}$ é conhecida. Esta LMI é factível se, e somente se, a matriz $A$ é estável, isto é, todas as trajetórias de (2.8) convergem para zero quando $t \rightarrow \infty$, ou equivalentemente, todos os autovalores tem parte real negativa. Esta LMI pode ser resolvida de modo explícito, ou seja, escolhe-se $Q>0$ e resolve-se a equação de Lyapunov (2.9), que é apenas um conjunto de $n(n+1) / 2$ equações lineares de $n(n+1) / 2$ variáveis escalares em $P$. Este conjunto de equações lineares terá solução e resultará em $P>0$ se, e somente se, a LMI for factível.

\subsubsection{Síntese de Controladores}

Em geral, no projeto de controladores deseja-se um desempenho do sistema em malha fechada que atenda vários objetivos de projeto, por exemplo, obter uma 
boa resposta transitória e ao mesmo tempo rejeitar perturbações com energia finita. Na teoria de controle, tal problema é denominado controle multi-objetivo ou misto. Muitas técnicas analíticas têm sido utilizadas na resolução de problemas de síntese, como a solução de equações de Riccati. Técnicas de projeto de controladores baseadas em LMIs vêm ganhando força pois podem atender a uma grande variedade de especificações de desempenho dentro de um simples problema de otimização LMI (Scherer et al., 1997).

Algumas aplicações de LMIs em projetos de controladores são descritas a seguir de forma resumida sem incluir a completa teoria envolvida no projeto dos controladores ou na demonstração dos teoremas de síntese.

\section{Estabilização por realimentação de estado}

O projeto de controladores para sistemas dinâmicos visa estabilizar o sistema em malha fechada e em geral satisfazer algumas restrições de desempenho. Quando o sinal de controle utiliza medições de todas as variáveis de estado diz-se que o controle é de realimentação de estado. Porém, nem sempre todo o vetor de estado é disponível para medição ou a utilização de muitos sensores pode ser economicamente inviável e na prática somente algumas informações estão disponíveis, chamadas variáveis de saída. Contudo, as propriedades da realimentação de estado são importantes e além disso é possível construir um controlador com realimentação de estado a partir das variáveis de saída, estimando-se as variáveis de estado através de filtros ou estimadores de estado.

Considere o sistema linear

$$
\dot{x}(t)=A x(t)+B u(t), x \in \mathbb{R}^{n}, u \in \mathbb{R}^{m} .
$$

O problema de estabilização consiste em determinar uma matriz $K \in \mathbb{R}^{m \times n}$ tal que a lei de controle linear

$$
u(t)=K x(t)
$$

estabilize assintoticamente o sistema em malha fechada

$$
\dot{x}(t)=(A+B K) x(t) .
$$


O sistema em malha fechada (2.13) é estável se, e somente se, existir uma matriz de Lyapunov $P=P^{T}>0$ tal que

$$
(A+B K)^{T} P+P(A+B K)<0 .
$$

Pré e pós multiplicando (2.14) por $P^{-1}$ e fazendo a mudança de variáveis $W=$ $P^{-1}$ e $Z=K W$ tem-se o seguinte resultado.

Lema 2.6 Existe $K$ tal que (2.13) é estável se, e somente se, existirem $W \in$ $\mathbb{R}^{n \times n}$ e $Z \in \mathbb{R}^{m \times n}$ tais que

$$
W>0, A W+W A^{T}+B Z+Z^{T} B^{T}<0
$$

onde $W=P^{-1}$ e $Z=K W$. Em caso afirmativo, o ganho é dado por $K=Z W^{-1}$.

\subsubsection{D-Estabilidade}

Para o sistema (2.8) ser exponencialmente estável todos os autovalores da matriz $A$ devem estar no semiplano esquerdo estrito. Porém, um sistema que seja exponencialmente estável pode apresentar um desempenho muito pobre devido a localização de seus pólos. A resposta temporal pode ser insatisfatória se, por exemplo, os pólos se localizarem muito próximo ao eixo imaginário e a parte imaginária de algum autovalor for muito maior que sua parte real (pólo oscilatório pouco amortecido). Por este motivo restringir que os pólos se encontrem em subregiões do semiplano esquerdo do plano complexo pode garantir sistemas mais rápidos e com poucas oscilações na resposta. Nesta seção apresenta-se brevemente como LMIs podem ser usadas para fornecer condições para alocar pólos em uma dada região $\mathcal{D}$ do plano complexo, isto é, alocar os autovalores de $A$ a uma região $\mathcal{D} \subset \mathbb{C}^{-}$. Mais detalhes podem ser encontrados em Chilali et al. (1999).

Definição 2.7 (D-Estabilidade) O sistema linear invariante (2.8) é D-estável se, e somente se, todos os autovalores da matriz $A \in \mathbb{R}^{n \times n}$ pertencem à sub-região $\mathcal{D}$ do plano complexo, isto é:

$$
\lambda_{i}(A) \in \mathcal{D} \subset \mathbb{C}^{-}, i=1, \ldots, n
$$


A noção de $\mathcal{D}$-estabilidade permite determinar não somente a estabilidade do sistema, mas também analisar o comportamento transitório deste que está diretamente relacionado à localização dos pólos no plano complexo. Note também que a estabilidade quadrática pode ser vista como um caso particular da $\mathcal{D}$ estabilidade quando $\mathcal{D}=\mathbb{C}^{-}$(Chilali et al., 1999).

A seguir apresenta-se uma classe de regiões do plano convexo descritas em termos de LMIs, proposta inicialmente por Chilali e Gahinet (1996).

Definição 2.8 (Região LMI) Um subconjunto $\mathcal{D}$ do plano complexo é denominado de região LMI se existem matrizes $L=L^{T} \in \mathbb{R}^{w \times w}$ e $M \in \mathbb{R}^{w \times w}$ tais que

$$
\mathcal{D}=\left\{s \in \mathbb{C}: L+s M+s^{*} M^{T}<0\right\},
$$

onde $s=\sigma+j \omega$.

Note que uma região LMI é um subconjunto do plano complexo representada por uma LMI em $s$ e $s^{*}$. As matrizes $L$ e $M$ em (2.15) detalham a região LMI no plano complexo de acordo com Definição 2.8. Segue abaixo alguns exemplos de regiões LMIs.

Exemplo 2.5 (Regiões LMIs) Considere as seguintes regiões LMIs representadas na Figura 2.2:

(a) $\mathcal{D}_{a}$, semi-plano com $\operatorname{Re}(s)<-\alpha$. Esta região pode ser definida pela desigualdade $s+s^{*}<-2 \alpha$, a qual pode ser representada na formulação LMI em (2.15) através das seguintes matrizes:

$$
L=2 \alpha \quad \text { e } \quad M=1 .
$$

(b) $\mathcal{D}_{b}$, disco com raio $r$ centrado $(-c, 0)$. Esta região pode ser definida pela seguinte relação $(\sigma+c)^{2}=\omega^{2}<r^{2}$ ou equivalentemente por $(s+c) r^{-1}\left(s^{*}+\right.$ c) $<r$ levando à seguinte definição das matrizes $L$ e $M$ em (2.15):

$$
L=\left[\begin{array}{cc}
-r & c \\
c & -r
\end{array}\right] \text { e } M=\left[\begin{array}{ll}
0 & 1 \\
0 & 0
\end{array}\right]
$$


(c) $\mathcal{D}_{c}$, setor cônico com ângulo interno $2 \psi$. Esta região pode ser definida por $\sigma \operatorname{sen} \psi+w \cos \psi<0$ levando às seguintes matrizes:

$$
L=\left[\begin{array}{cc}
0 & 0 \\
0 & 0
\end{array}\right] \text { e } M=\left[\begin{array}{cc}
\operatorname{sen} \psi & \cos \psi \\
-\cos \psi & \operatorname{sen} \psi
\end{array}\right]
$$

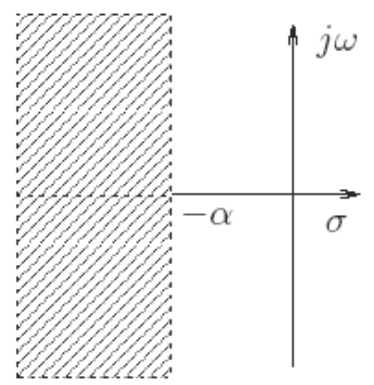

(a)

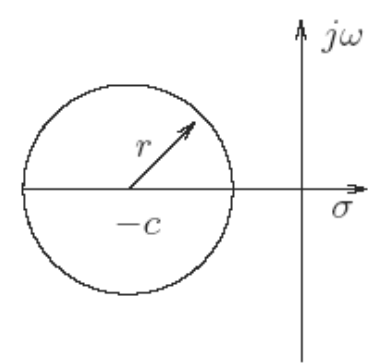

(b)

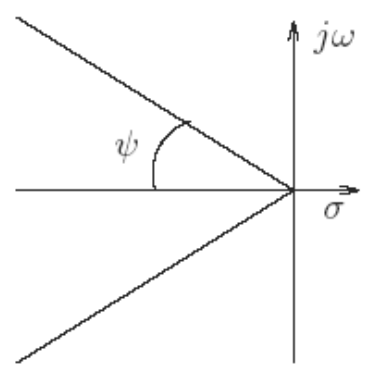

(c)

Figura 2.2: Regiões LMIs - (a) semi-plano, (b) disco e (c) setor cônico.

O seguinte resultado fornece uma condição LMI para determinar a $\mathcal{D}$-estabilidade.

Lema 2.9 (Chilali et al., 1999) O sistema (2.8) é D-estável se existe uma matriz simétrica positiva definida $P$ tal que:

$$
L \otimes P+M \otimes(P A)+M^{T} \otimes\left(A^{T} P\right)<0
$$

onde o operador $\otimes$ corresponde ao produto de Kronecker ${ }^{1}$ de duas matrizes.

Além da análise de $\mathcal{D}$-estabilidade que indica se os autovalores estão na região procurada do plano complexo, segue o projeto de controladores que levem os pólos às regiões desejadas.

Considere a forma dual de (2.17),

$$
L \otimes Q+M \otimes(A Q)+M^{T} \otimes\left(Q A^{T}\right)<0
$$

$\operatorname{com} Q=P^{-1}$.

A forma dual (2.18) para a $\mathcal{D}$-estabilidade permite a proposição do seguinte resultado.

\footnotetext{
${ }^{1}$ Para duas matrizes $A$ e $B$ o produto de Kronecker é dado por $A \otimes B=\left[A_{i j} B\right]_{i j}$.
} 
Teorema 2.10 Considere o sistema (2.13) e uma região LMI $\mathcal{D}$ contida em $\mathbb{C}^{-}$. Suponha que as matrizes $Q$ e $Y$ de dimensões apropriadas sejam uma solução do seguinte problema LMI

$$
Y, Q>0: L \otimes Q+M \otimes(A Q+B Y)+M^{T} \otimes\left(Q A^{T}+Y^{T} B^{T}\right)<0
$$

Então, o sistema (2.13) com $K=Y Q^{-1}$ é D-estável. Em outras palavras

$$
\lambda_{i}(A+B K) \in \mathcal{D} \subset \mathbb{C}^{-}, \quad i=1, \ldots, n
$$

Prova: Obtém-se (2.19) utilizando a lei de realimentação de estado (2.12) em (2.11), substituindo $A$ por $A+B K$ em (2.18) e fazendo $Y=K Q$.

Para o caso particular de alocação dos pólos em uma região circular do plano-s é possível utilizar o seguinte resultado.

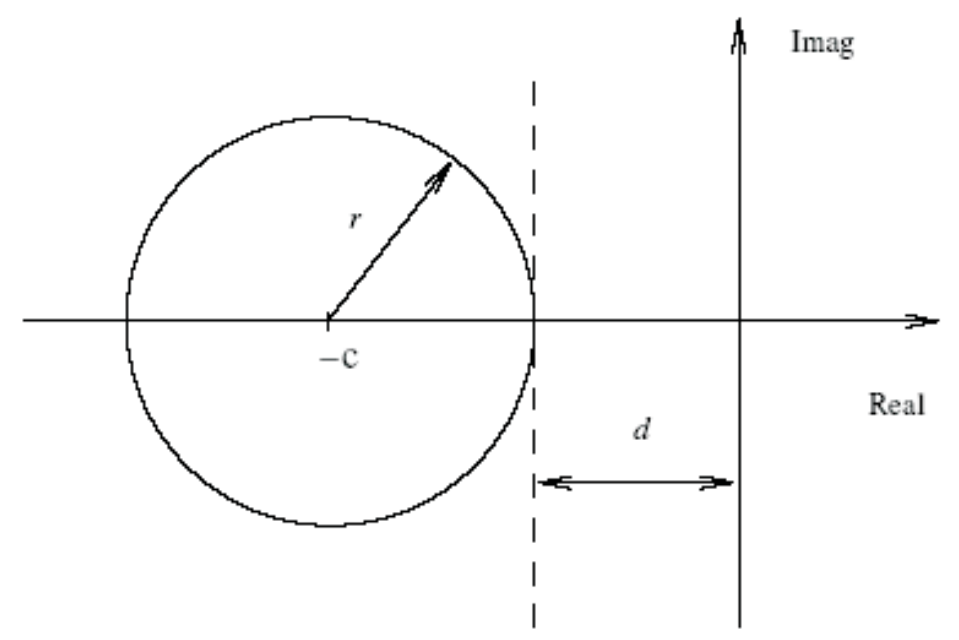

Figura 2.3: Região circular para alocação de pólos.

Corolário 2.11 Considere uma região LMI circular $\mathcal{D} \subset \mathbb{C}^{-}$. O sistema (2.11) é $\mathcal{D}$-estável se existir uma matriz $Z$ e uma matriz simétrica definida positiva $P$, de dimensões apropriadas, tais que ${ }^{2}$

$$
\left[\begin{array}{cc}
\bar{A} P+P \bar{A}^{T}+B Z+Z^{T} B^{T} & * \\
P \bar{A}^{T}+Z^{T} B^{T} & -r P
\end{array}\right]<0,
$$

\footnotetext{
${ }^{2} \mathrm{O}$ símbolo $*$ denota o transposto do bloco diagonalmente oposto.
} 
onde $\bar{A}=A+d I$ e $d=c-r$, conforme ilustra a Figura 2.3. O controlador é dado por $K=Z P^{-1}$.

Prova: A partir do Lema 2.9 e da forma dual (2.18), utilizando as matrizes $L$ e $M$ como em (2.16), tem-se

$$
\begin{aligned}
& {\left[\begin{array}{cc}
-r P & c P \\
c P & -r P
\end{array}\right]+\left[\begin{array}{cc}
0 & A P \\
0 & 0
\end{array}\right]+\left[\begin{array}{cc}
0 & 0 \\
P A^{T} & 0
\end{array}\right]<0,} \\
& {\left[\begin{array}{cc}
-r P & c P+A P \\
c P+P A^{T} & -r P
\end{array}\right]<0 .}
\end{aligned}
$$

Aplicando o complemento de Schur, tem-se

$$
-r P+(c P+A P)(r P)^{-1}(c P+A P)^{T}<0 .
$$

Manipulando a desigualdade acima chega-se a

$$
(A+d I) P+P(A+d I)^{T}+\frac{1}{r}(A+d I) P(A+d I)^{T}<0 .
$$

Aplicando novamente o complemento de Schur tem-se,

$$
\left[\begin{array}{cc}
\bar{A} P+P \bar{A}^{T} & \bar{A} P \\
P \bar{A}^{T} & -r P
\end{array}\right]<0,
$$

$\operatorname{com} \bar{A}=A+d I$. Substituindo na LMI (2.22) a matriz de estado em malha aberta $\bar{A}$ pela matriz de malha fechada $\bar{A}+B K$ e fazendo $Z=K P$, chega-se a $(2.20)$.

A LMI (2.21) também pode ser obtida através da desigualdade do sistema discreto. No sistema discreto, se existir uma matriz simétrica definida positiva $P$, que satisfaça

$$
A P A^{T}-P<0
$$

garante que os autovalores de $A$ pertençam a região de círculo unitário centrada na origem. Para que os autovalores da matriz de estado possam pertencer a região circular de raio $r$ e centro em $(-c, 0)$ basta substituir $A$ por $\frac{A+c I}{r}$ e $c=r+d$ em (2.23). 
Exemplo 2.6 (Alocação de pólos em um circulo) Considere o sistema contínuo no tempo

$$
\dot{x}(t)=A x(t)+B u(t)
$$

onde

$$
A=\left[\begin{array}{cc}
2.439 & 0.683 \\
0.933 & -2.787
\end{array}\right] \text { e } B=\left[\begin{array}{l}
0.607 \\
0.629
\end{array}\right]
$$

Note que A é instável com autovalores em 2.56 e -2.91. Deseja-se, através de uma lei de realimentação de estado $u=K x(t)$, posicionar os pólos em um círculo de raio 3 e centro em $(-10,0)$.

Através da resolução da LMI (2.19), com o uso do aplicativo Matlab, obteve-se

$$
\begin{aligned}
& P=\left[\begin{array}{cc}
0.656 & 1.21 \\
1.21 & 2.33
\end{array}\right], \\
& Q=\left[\begin{array}{ll}
-14.78 & -26.19
\end{array}\right],
\end{aligned}
$$

e o controlador

$$
K=\left[\begin{array}{ll}
-40.54 & 9.79
\end{array}\right]
$$

Os autovalores do sistema em malha fechada foram alocados em -9.96 e -8.84 , satisfazendo a localização desejada.

Utilizando a LMI (2.20) obteve-se resultado similar

$$
\begin{aligned}
& P=\left[\begin{array}{ll}
1.92 & 3.85 \\
3.85 & 8.06
\end{array}\right], \\
& Q=\left[\begin{array}{ll}
-39.2 & -76.13
\end{array}\right],
\end{aligned}
$$

e o controlador

$$
K=\left[\begin{array}{ll}
-35.18 & 7.35
\end{array}\right]
$$

Os autovalores do sistema em malha fechada foram alocados em $-8.54+j 1.18$ e -8.54-j1.18, também satisfazendo a localização desejada. A região de restrição $\mathcal{D}$ é grande para acomodar respostas diferentes para este problema de factibilidade. 
A resposta temporal é ilustrada na Figura 2.4. Pode-se observar que o primeiro controlador obtido posiciona os pólos mais a esquerda no plano-s, o que pode ser verificado pelo comportamento ligeiramente mais agressivo e com maior amplitude do sinal de saída $u(t)$.
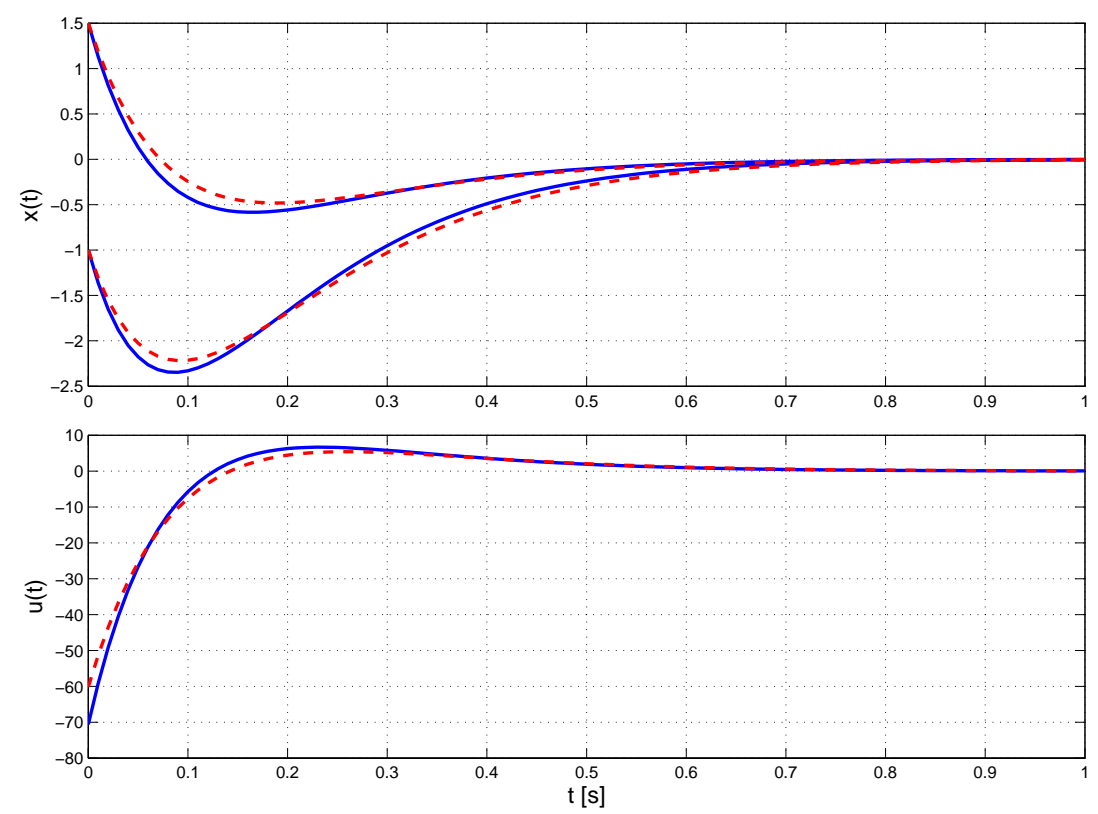

Figura 2.4: Resposta do sistema em malha fechada para a condição inicial $x_{0}^{T}=[1.5-1]$. A linha contínua corresponde ao controlador obtido pela resolução da LMI (2.19) e a linha tracejada ao controlador obtido da LMI (2.20). 


\section{Capítulo 3}

\section{Sistemas Fuzzy}

\subsection{Modelagem Fuzzy Takagi-Sugeno}

O modelo fuzzy proposto por Takagi e Sugeno é descrito por regras fuzzy SEENTÃO que representam relações lineares entre a entrada e a saída de um sistema não-linear (Tanaka e Wang, 2001). A principal característica de um modelo fuzzy T-S é expressar a dinâmica local de cada implicação fuzzy (regra) por um modelo de sistema linear (afim). O modelo fuzzy global é obtido pela combinação dos modelos lineares (afins) locais descritos por regras do tipo

\section{Regra de Modelo $i$ :}

SE $z_{1}(t)$ é $\mathcal{M}_{i 1}$ e $\ldots$ e $z_{p}(t)$ é $\mathcal{M}_{i p}$

ENTÃO

$$
\left\{\begin{array}{l}
\dot{x}(t)=A_{i} x(t)+B_{i} u(t) \\
y(t)=C_{i} x(t), \quad i=1,2, \ldots, r
\end{array}\right.
$$

no qual $\mathcal{M}_{i j}$ é o $i j$-ésimo conjunto fuzzy e r é o número de regras de modelo; $x(t) \in \mathbb{R}^{n}$ é o vetor de estados, $u(t) \in \mathbb{R}^{m}$ é o vetor de entrada, $y(t) \in \mathbb{R}^{q}$ é o vetor de saída, $A_{i} \in \mathbb{R}^{n \times n}, B_{i} \in \mathbb{R}^{n \times m}$, e $C_{i} \in \mathbb{R}^{q \times n} ; z_{1}(t), \ldots, z_{p}(t)$ são variáveis premissas que podem ser funções das variáveis de estado, de distúrbios externos ou do tempo. Dado um par $(x(t), u(t))$, a saída final do sistema fuzzy é a média ponderada dos modelos fuzzy locais: 


$$
\begin{aligned}
\dot{x}(t) & =\frac{\sum_{i=1}^{r} w_{i}(z(t))\left\{A_{i} x(t)+B_{i} u(t)\right\}}{\sum_{i=1}^{r} w_{i}(z(t))} \\
& =\sum_{i=1}^{r} \mu_{i}(z(t))\left\{A_{i} x(t)+B_{i} u(t)\right\} \\
& =A(\mu) x(t)+B(\mu) u(t), \\
y(t) & =\frac{\sum_{i=1}^{r} w_{i}(z(t)) C_{i} x(t)}{\sum_{i=1}^{r} w_{i}(z(t))} \\
= & \sum_{i=1}^{r} \mu_{i}(z(t)) C_{i} x(t) \\
= & C(\mu) x(t),
\end{aligned}
$$

sendo

$$
\begin{aligned}
& z(t)=\quad\left[z_{1}(t) z_{2}(t) \ldots z_{p}(t)\right] \\
& w_{i}(z(t))=\prod_{j=1}^{p} M_{i j}(z(t)) \\
& \mu_{i}(z(t))=\frac{w_{i}(z(t))}{\sum_{i=1}^{r} w_{i}(z(t))}
\end{aligned}
$$

para todo $t$. O termo $M_{i j}(z(t))$ é o grau de pertinência de $z_{j}(t)$ em $\mathcal{M}_{i j}$, ou seja, expressa o quanto a variável premissa $z_{j}(t)$ pertence ao conjunto fuzzy $\mathcal{M}_{i j}$ no tempo t. Esta é a principal diferença entre a teoria de conjuntos fuzzy e a teoria de conjuntos convencionais, que apenas pode dizer se determinado elemento pertence ou não a certo conjunto. Cada conjunto fuzzy pode ser associado a uma função pertinência $M_{i j}\left(z_{j}(t)\right)$. As funções de pertinência podem ser triangulares, trapezoidais, gaussianas, ou qualquer outro formato. O peso associado a cada $i$-ésima regra calculado das funções de pertinência é dado por $w_{i}(z(t))$.

E desde que

$$
\left\{\begin{array}{l}
\sum_{i=1}^{r} w_{i}(z(t))>0 \\
w_{i}(z(t)) \geq 0, \quad i=1,2, \ldots, r
\end{array}\right.
$$


tem-se

$$
\left\{\begin{array}{l}
0 \leq \mu_{i}(z(t)) \leq 1 \\
\sum_{i=1}^{r} \mu_{i}(z(t))=1, \quad i=1, \ldots, r .
\end{array}\right.
$$

\subsubsection{Linearização de Dinâmicas Não-Lineares}

Nem sempre os modelos fuzzy Takagi-Sugeno são fáceis de interpretar. Idealmente, é desejado que a dinâmica do modelo global fuzzy T-S seja uma boa aproximação do sistema não-linear e ao mesmo tempo os modelos lineares locais do modelo fuzzy sejam aproximações precisas da linearização local da dinâmica não-linear. Para muitas aplicações é mais importante aproximar o modelo global fuzzy do sistema original quando, por exemplo, o modelo global é utilizado para predição ou quando ele é utilizado como um modelo interno de um controlador. Há casos porém, que a precisão dos modelos locais deve ser considerada, como no caso de se utilizar o modelo fuzzy T-S para a síntese de controladores, pois os modelos locais são utilizados para projetar os controladores lineares locais. Temse então um problema multi-objetivo de construção de um modelo que seja uma boa aproximação de ambas as dinâmicas locais e globais (Johansen et al., 2000).

Embora, estes objetivos possam ser conflitantes em alguns casos, pode-se mostrar que existe uma relação próxima entre o modelo fuzzy T-S e a linearização dinâmica quando utilizadas estruturas de modelos locais afins. Contudo, a estrutura afim é sensível à parametrização em regimes de operação transitórios, isto é, distante dos pontos de equilíbrio. A razão é essencialmente devido ao fato de que o termo constante do modelo afim tende a dominar a dinâmica em relação ao termo linear nesta região. Então, a escolha de modelos lineares, nos regimes transitórios, pode apresentar algumas vantagens sobre o modelo afim apesar de possuir, em geral, uma vizinhança de validade menor. Além disso, é mais complexo avaliar as informações dos modelos afastados dos pontos de equilíbrio do sistema.

A alocação dos autovalores de um modelo linear local, na região de regime de transição, garante apenas que a convergência das trajetórias, em relação a uma 
trajetória nominal, tem decaimento exponencial. Porém, nada pode-se afirmar quanto a estabilidade dos pontos de equilíbrio do sistema não-linear. Isto porque não há sentido em falar em estabilidade de ponto de equilíbrio para esta região, visto que o ponto de equilíbrio deste sistema não coincide com o do sistema não-linear. Neste contexto, utiliza-se, em geral, a idéia de estabilidade de uma trajetória, ou seja, uma trajetória com uma pequena pertubação em relação a uma trajetória nominal se aproximará a esta com o passar do tempo. Para um modelo afim a alocação apropriada dos autovalores de $A$ pode ter menor importância na dinâmica das trajetórias em relação ao termo afim.

\section{Linearização Dinâmica}

O modelo dinâmico T-S é composto de múltiplos modelos dinâmicos lineares (afins). Seria desejável, do ponto de vista de interpretação, análise e aplicação, que estes sistemas locais estivessem relacionados as linearizações do sistema nãolinear. Segue uma breve revisão da linearização dinâmica para melhor entendimento.

Suponha que o modelo verdadeiro da planta seja da forma

$$
\dot{x}(t)=f(x(t))+G(x(t)) u(t)
$$

onde $f$ e $G$ são funções suaves. Por simplificação define-se $F(x, u):=f(x)+$ $G(x) u$. Então, o modelo pode ser representado por $\dot{x}=F(x, u)$.

No ponto de equilíbrio $\left(x_{0}, u_{0}\right)$ a linearização local é dada por

$$
\dot{x}=F\left(x_{0}, u_{0}\right)+\frac{\partial F}{\partial x}\left(x_{0}, u_{0}\right)\left(x-x_{0}\right)+\frac{\partial F}{\partial u}\left(x_{0}, u_{0}\right)\left(u-u_{0}\right)+\text { t.a.o },
$$

no qual t.a.o denota os termos de alta ordem.

Desconsiderando os termos de alta ordem da expansão em série de Taylor (3.6) na qual $\left(x_{0}, u_{0}\right)$ é um ponto de equilíbrio, ou seja, $F\left(x_{0}, u_{0}\right)=0$, tem-se

$$
\dot{x}=A\left(x_{0}, u_{0}\right)\left(x-x_{0}\right)+B\left(x_{0}, u_{0}\right)\left(u-u_{0}\right),
$$


onde

$$
\begin{aligned}
A\left(x_{0}, u_{0}\right) & =\frac{\partial F}{\partial x}\left(x_{0}, u_{0}\right) \\
B\left(x_{0}, u_{0}\right) & =\frac{\partial F}{\partial u}\left(x_{0}, u_{0}\right) .
\end{aligned}
$$

A linearização local (3.7) representa o comportamento do sistema não-linear (3.5) na vizinhança de $\left(x_{0}, u_{0}\right)$. Considerando a origem o ponto de equilíbrio, é possível reescrever $(3.6)$ em torno de $(0,0)$ como

$$
\dot{x}=A\left(x_{0}, u_{0}\right) x+B\left(x_{0}, u_{0}\right) u \text {. }
$$

Para um ponto de equilíbrio $\left(x_{0}, u_{0}\right)$ qualquer, o modelo local não é linear em relação a $x$ e a $u$ devido ao termo constante $-\left(A x_{0}+B u_{0}\right)$, portanto a linearização de Taylor apenas resultará em um sistema linear em $x$ e em $u$ para o ponto de equilíbrio $\left(x_{0}, u_{0}\right)=(0,0)$.

A seguir, considera-se um caso mais geral onde a linearização de (3.5) é calculada sobre uma trajetória arbitrária $\left(x_{0}(t), u_{0}(t)\right)$. Esta trajetória é definida pelo estado inicial $x_{0}(0)$ e satisfaz $\dot{x}_{0}=f\left(x_{0}, u_{0}\right)$. A linearização da trajetória $\left(x_{0}(t), u_{0}(t)\right)$, ou seja, a linearização dinâmica de (3.5) em torno de $\left(x_{0}(t), u_{0}(t)\right)$, desprezando os termos de alta ordem, é dada pelo sistema variante no tempo (LTV, do inglês Linear Time Variant)

$\dot{x}(t)=F\left(x_{0}(t), u_{0}(t)\right)+A\left(x_{0}(t), u_{0}(t)\right)\left(x(t)-x_{0}(t)\right)+B\left(x_{0}(t), u_{0}(t)\right)\left(u(t)-u_{0}(t)\right)$,

onde as funções $A$ e $B$ são definidas como em (3.8) e (3.9).

A análise de estabilidade de uma trajetória de um sistema autônomo pode ser reduzida a análise de um sistema linearizado não-autônomo sobre um ponto de equilíbrio (Bontempi e Birattari, 2005). Para simplificar, considere o sistema (3.5) com entrada nula $u(t) \equiv 0$ com uma solução $x^{*}(t)$, com condição inicial $x^{*}(0)=x_{0}^{*}$.

Considere uma perturbação na condição inicial $x(0)=x_{0}^{*}+\delta x_{0}$ e o erro

$$
\tilde{x}(t)=x(t)-x^{*}(t)
$$


Desde que $x^{*}(t)$ e $x(t)$ são soluções de $(3.5)$, tem-se que $\tilde{x}(t)$ satisfaz a seguinte equação diferencial não-autônoma

$$
\dot{\tilde{x}}(t)=g(\tilde{x}, t)
$$

cujo equilíbrio é a origem. Então, cada trajetória nominal do sistema autônomo corresponde a um sistema não autônomo equivalente. Seja $A(t)$ a linearização do sistema (3.11) em torno do equilíbrio 0. Tem-se o seguinte teorema.

Teorema 3.1 (Bontempi e Birattari, 2005) Se o sistema linearizado A(t) é uniformemente assintoticamente estável, então o ponto de equilíbrio 0 do sistema não-autônomo (3.11) é também uniformemente assintoticamente estável.

Se 0 é um ponto de equilíbrio estável de (3.11) então $\tilde{x}(t)$ tende a zero e, portanto, a trajetória $x(t)$ se aproxima assintoticamente da trajetória nominal $x^{*}(t)$.

A aproximação do sistema pela modelagem fuzzy Takagi-Sugeno é dada a seguir

$$
\dot{x}(t)=\sum_{i=1}^{N} \mu_{i}(x(t), u(t))\left\{F\left(x_{i}, u_{i}\right)+A\left(x_{i}, u_{i}\right)\left(x(t)-x_{i}\right)+B\left(u(t)-u_{i}\right)\right\} .
$$

Seja $Z^{0}=\left\{\left(x_{1}, u_{1}\right),\left(x_{2}, u_{2}\right), \ldots,\left(x_{N}, u_{N}\right)\right\} \subset Z=X \times U$ o conjunto dos pontos de linearização de (3.12). Intuitivamente espera-se que (3.12) se aproxime de (3.10) quando $Z^{0}$ se aproxima de $Z$, ou seja, $N \rightarrow \infty$.

\section{Interpretação dos Modelos Locais em Regimes de Operação de Transição}

Modelos locais em regimes de operação de transição são modelos que não possuem, por definição, pontos de equilíbrios do sistema não-linear na sua região de validade. Entretanto, tais modelos possuem pontos de equilíbrio denominados pontos de equilíbrio virtuais (Johansen et al., 2000). Estes são chamados assim porque não possuem nenhuma relação com os pontos de equilíbrio do sistema não-linear. Para estes modelos a análise de autovalores não fornece necessariamente nenhuma informação sobre a estabilidade local dos pontos de equilíbrio do sistema não-linear. 
Na Figura 3.1 estão ilustrados os pontos de equilíbrios virtuais de dois modelos distintos para uma mesma região do sistema não-linear. Pode ser observado que as trajetórias são governadas pela combinação dos modelos locais mostrando que o campo vetorial do sistema não-linear pode ser caracterizado com precisão por diversos tipos de modelos.

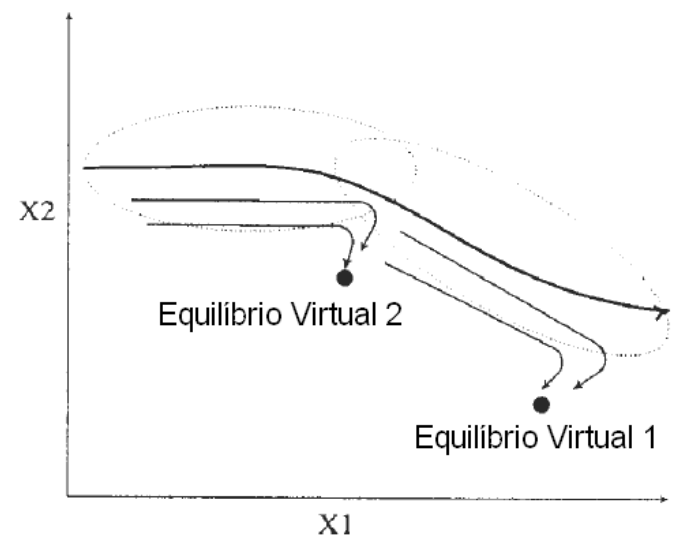

(a)

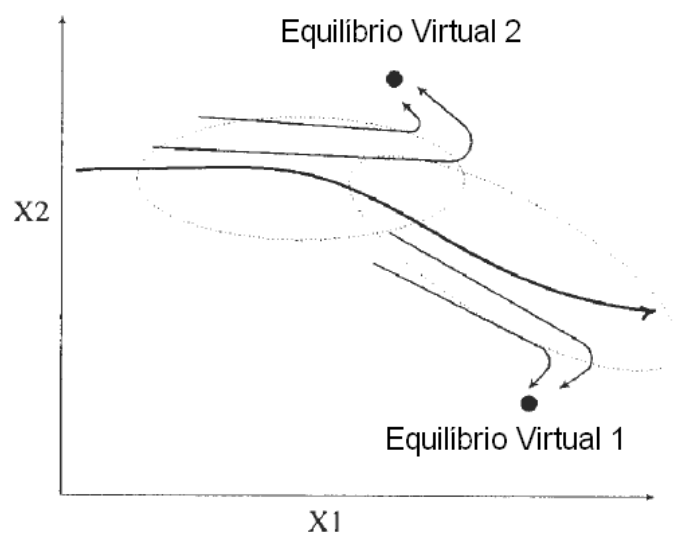

(b)

Figura 3.1: Trajetória do modelo global composta por dois modelos locais. Os modelos locais (a) e (b) são distintos, porém, resultam na mesma trajetória.

Seja uma representação afim da forma

$$
\dot{x}=A_{i}\left(x-x_{i}\right)+B_{i}\left(u-u_{i}\right)+d_{i}
$$

uma aproximação válida de uma pequena vizinhança do ponto $\left(x_{i}, u_{i}\right)$. A estrutura (3.13) contém muitos graus de liberdade pois, em muitos casos, a constante 
$d_{i}$ pode dominar e os parâmetros $A_{i}$ e $B_{i}$ possuirem menor efeito. Suponha

$$
d_{i}=F\left(x_{i}, u_{i}\right)
$$

Os parâmetros $A_{i}$ e $B_{i}$ podem ser escolhidos de diversas formas (Johansen et al., 2000):

- $A_{i}$ e $B_{i}$ podem ser selecionados de modo que (3.13) seja uma representação exata da linearização de $(3.5)$ no ponto $\left(x_{i}, u_{i}\right)$, ou seja, $A_{i} \approx A\left(x_{i}, u_{i}\right)$ e $B_{i} \approx B\left(x_{i}, u_{i}\right)$. Isto é vantajoso em termos de interpretação, análise e aplicabilidade do modelo em projeto de controle mas tem a desvantagem de levar a uma menor região de validade do modelo local.

- $A_{i}$ e $B_{i}$ podem ser selecionados de forma a aumentar a região de validade de (3.13) e/ou aumentar a precisão do modelo global. Neste caso, os parâmetros podem ser completamente diferentes aos da linearização.

Para o caso de escolha de um modelo local linear em regiões de regime de transição também há várias possibilidades. $A_{i}$ pode ser obtido como em (3.8), o que leva $B_{i}$ assumir um valor diferente de (3.9), de forma a se obter um modelo exato no ponto de operação. Outra alternativa seria escolher ambos os parâmetros de forma a coincidir com a linearização, porém, não se obteria uma representação precisa no ponto de operação. A opção detalhada na próxima seção é a desenvolvida por Teixeira e Zak (1999), que através da resolução de um problema de otimização busca o melhor modelo linear com o menor erro de aproximação.

Como exemplo de modelagem local de um sistema dinâmico considere as duas representações abaixo de um sistema não-linear em torno de um ponto de operação:

$$
\begin{aligned}
& \sum_{1}: \dot{x}=A_{1} x \\
& \sum_{2}: \dot{x}=A_{2} x+d,
\end{aligned}
$$

onde

$$
A_{1}=\left[\begin{array}{cc}
4 & -4.5 \\
4.05 & -4.55
\end{array}\right] \quad A_{2}=\left[\begin{array}{cc}
0.51 & -4.29 \\
3.84 & -4.51
\end{array}\right]
$$


e

$$
d=\left[\begin{array}{l}
-8.58 \\
-0.27
\end{array}\right]
$$

O campo vetorial de ambos os sistemas $\sum_{1}$ e $\sum_{2}$ é descrito respectivamente nas Figuras 3.2 e 3.3. Os sistemas são qualitativamente diferentes, enquanto o primeiro é um nó estável com ponto de equilíbrio na origem o segundo é um foco estável com ponto de equilíbrio um pouco deslocado da origem.

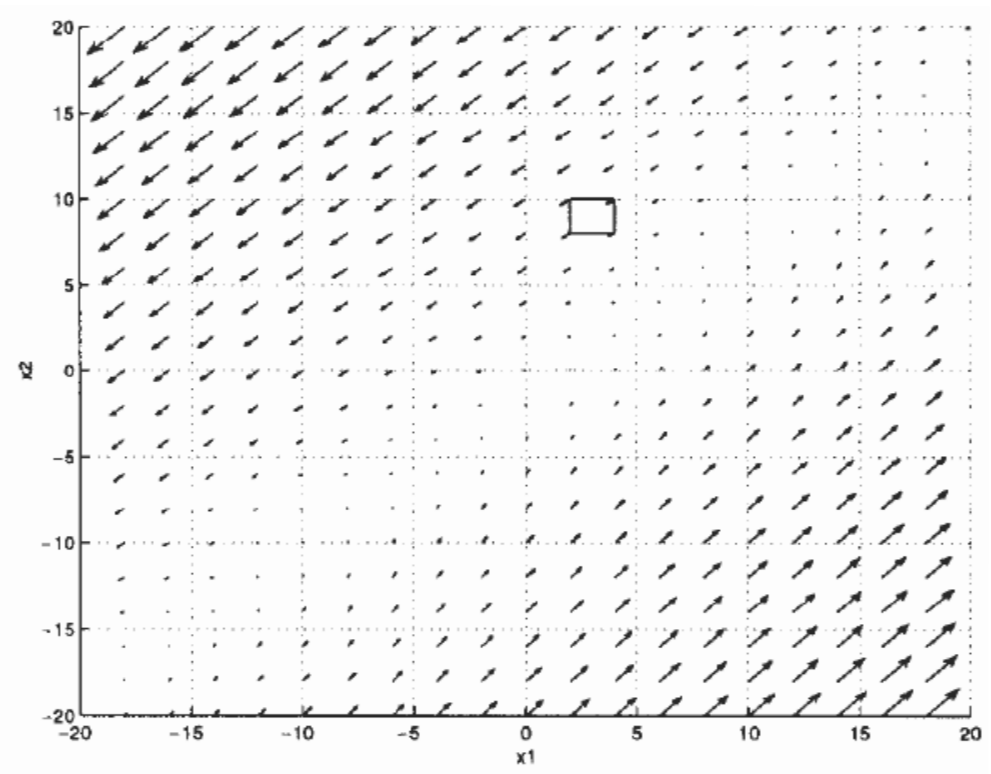

Figura 3.2: Campo vetorial do sistema $\sum_{1}$.

Contudo, na região definida por $\mathcal{R}=\left\{\left(x_{1}, x_{2}\right): 2 \leq x_{1} \leq 4\right.$ e $\left.8 \leq x_{2} \leq 10\right\}$ o fluxo é similar, conforme mostram as Figuras 3.4 e 3.5. Pode-se concluir então que na região $\mathcal{R}$, sujeito a algum erro de aproximação, as dinâmicas definidas por $\sum_{1}$ e $\sum_{2}$ são equivalentes e são representações válidas do sistema não-linear. Porém, na região fora de $\mathcal{R}$ a representação não é mais válida. Em geral, a análise qualitativa dos modelos locais que não contenham o ponto de equilíbrio do sistema não-linear pode dizer muito pouco sobre o sistema original devido ao fato de ser válido apenas naquela região (Shorten et al., 1999), conforme ilustra o exemplo acima. 


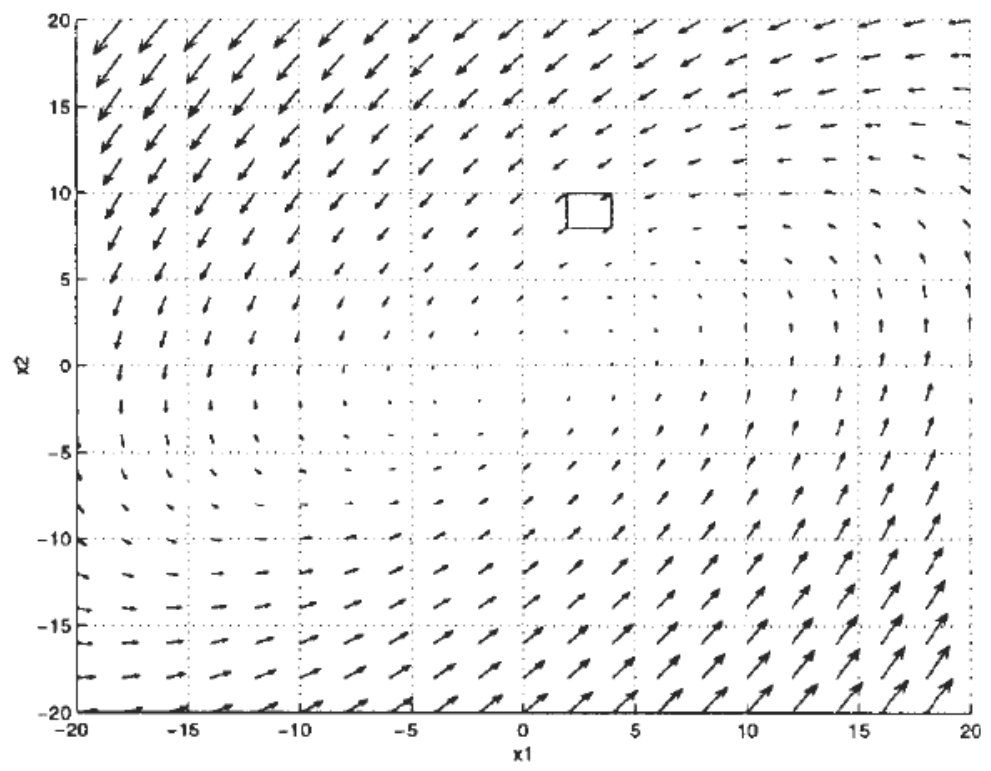

Figura 3.3: Campo vetorial do sistema $\sum_{2}$.

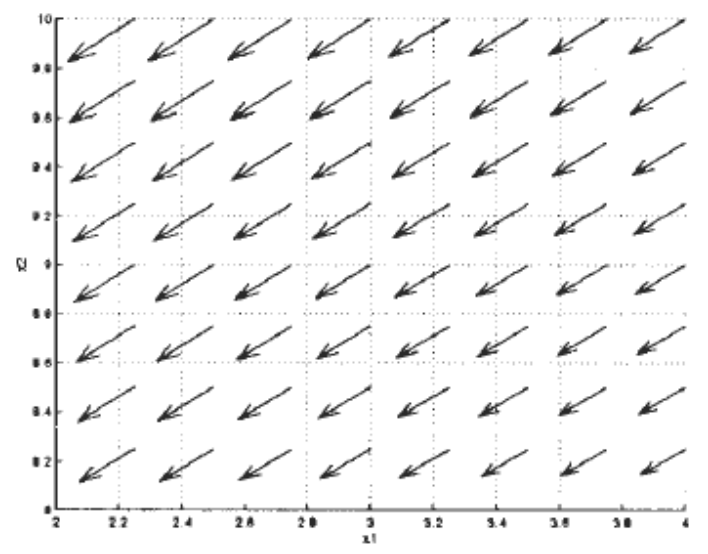

Figura 3.4: Campo vetorial de $\sum_{1}$ na região $R$.

\subsubsection{Aproximação de Sistemas Não-Lineares por Siste- mas Lineares Fuzzy T-S}

Modelos dinâmicos fuzzy Takagi-Sugeno podem ser modelo lineares locais, modelos lineares afins ou constantes locais. Um modelo fuzzy T-S geral emprega um modelo afim com um termo constante para cada regra. Neste trabalho será focado um caso particular do modelo geral, no qual cada regra é composta por um modelo linear. 


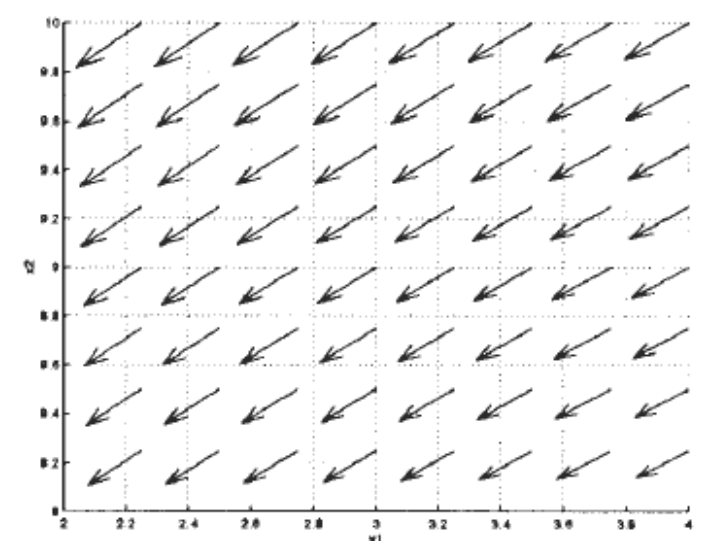

Figura 3.5: Campo vetorial de $\sum_{2}$ na região $R$.

É conhecido que sistemas com dinâmicas não-lineares suaves podem ser bem aproximados por modelos fuzzy T-S com modelos locais afins (Cao et al., 1996). Porém, muitos resultados já desenvolvidos em estabilidade e síntese de modelos fuzzy T-S são baseados em regras com modelos lineares. O seguinte resultado de Wang et al. (2000) mostra que modelos locais lineares fuzzy T-S podem ser aproximadores universais de dinâmicas não-lineares suaves.

Teorema 3.2 (Wang et al., 2000) Qualquer sistema não-linear suave $\dot{x}=f(x)$ satisfazendo $f(0)=0$ e $f \in \mathbb{C}_{n}^{2}$, pode ser aproximado, com qualquer grau de precisão, por um modelo fuzzy $T-S$ da forma $\dot{x}=\hat{f}(x)=\sum_{i=1}^{r} \mu_{i}(x) A_{i} x$.

Não há perda de generalidade na condição $f(0)=0$ pois no caso de $f(0) \neq$ $0, f$ pode ainda ser aproximado por um modelo linear T-S através de uma simples mudança de coordenadas. Similarmente, um sistema não-linear suave $\dot{x}=f(x)+g(x) u$ pode ser também aproximado por um modelo fuzzy T-S $\dot{x}=\sum_{i=1}^{r} \mu_{i}(x)\left(A_{i} x+B_{i} u\right)$. Um exemplo é dado para ilustração.

Exemplo 3.1 (Aproximação de funções com modelos fuzzy T-S) Considere a função não-linear de duas dimensões

$$
f\left(x_{1}, x_{2}\right)=8 x_{1}+10 x_{2} \operatorname{sen}\left(4 x_{1}\right)+x_{1}^{3}-4 x_{1} x_{2}
$$

como mostrado na Figura 3.6. Um modelo fuzzy T-S construído com modelos locais lineares, é ilustrado na Figura 3.7. O máximo erro de aproximação é 1, 38 
e foi plotado como mostra a Figura 3.8. Este erro pode ser reduzido aumentandose o número de regras fuzzy.

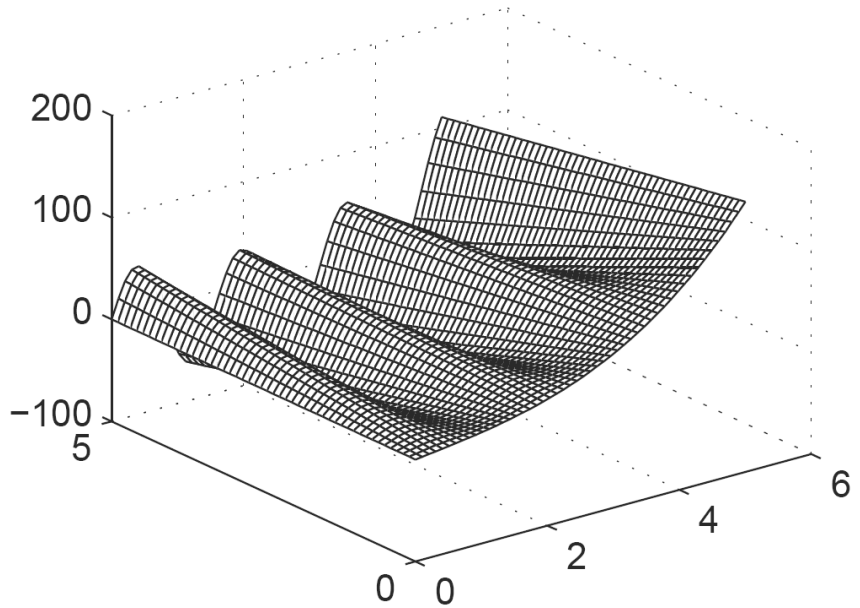

Figura 3.6: Função não-linear $f\left(x_{1}, x_{2}\right)=8 x_{1}+10 x_{2} \operatorname{sen}\left(4 x_{1}\right)+x_{1}^{3}-4 x_{1} x_{2}$.

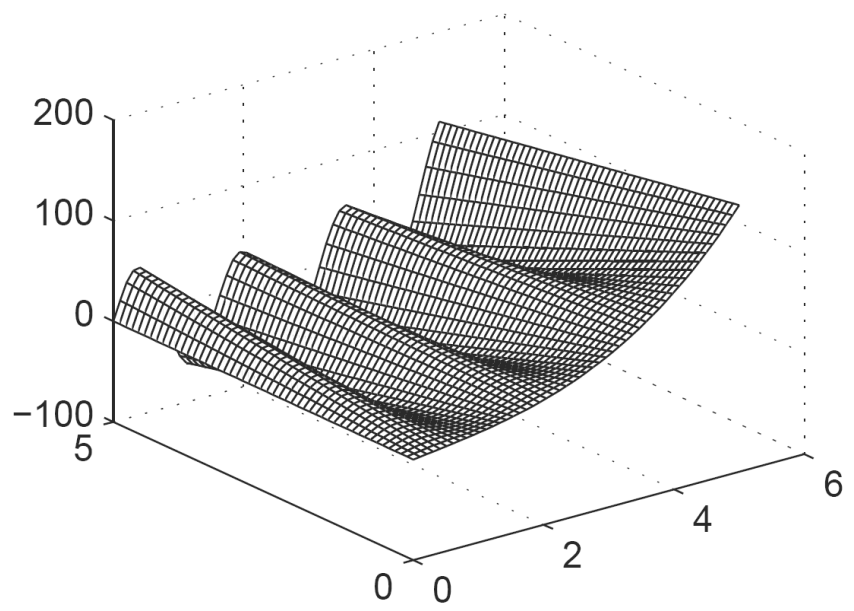

Figura 3.7: Modelo fuzzy T-S construído.

\section{Construção de Modelos Lineares Locais do Modelo Fuzzy Takagi-Sugeno}

Descreve-se a seguir um método sistemático para construir modelos locais de uma planta não-linear (Teixeira e Zak, 1999). Considera-se o modelo (3.5) e um ponto de operação $x_{0}$ que não precisa ser necessariamente um ponto de equilíbrio de (3.5). O objetivo é construir um modelo linear em $x$ e em $u$ que se aproxime ao comportamento de (3.5) na proximidade do ponto de operação $x_{0}$, isto é, achar 


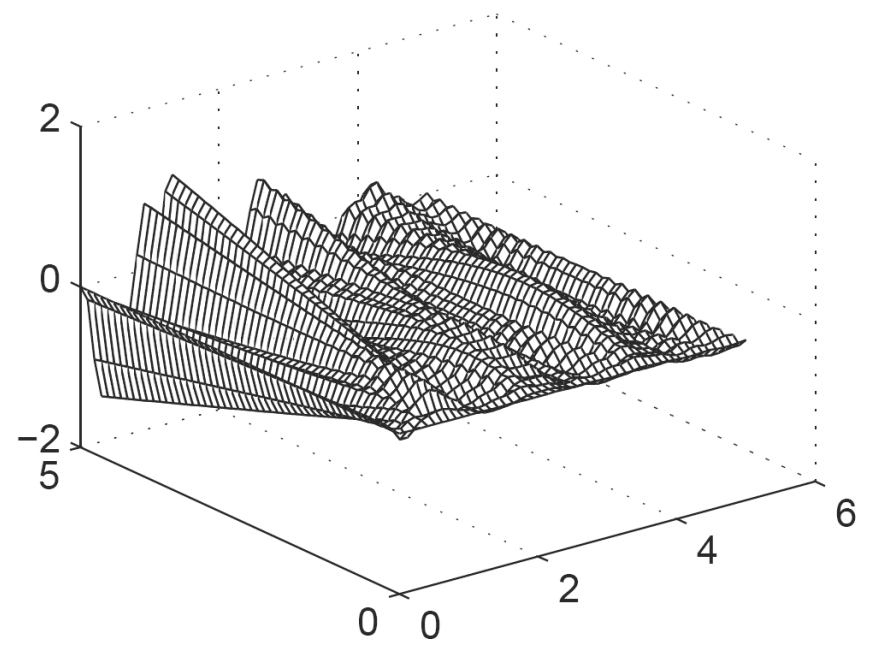

Figura 3.8: Erro de aproximação da função não-linear pelo modelo fuzzy T-S.

um $A$ e um $B$ tal que, na vizinhança de $x_{0}$,

$$
f(x)+G(x) u \approx A x+B u \text { para qualquer } u
$$

e

$$
f\left(x_{0}\right)+G\left(x_{0}\right) u=A x_{0}+B u \text { para qualquer } u \text {. }
$$

Portanto $G\left(x_{0}\right)=B$. Então, deve-se encontrar uma matriz $A$ tal que

$$
f(x) \approx A x
$$

e

$$
f\left(x_{0}\right)=A x_{0}
$$

Seja $a_{i}^{T}$ a $i$-ésima linha da matriz $A$. Então, (3.16) pode ser representado por:

$$
f_{i}(x) \approx a_{i}^{T} x, \quad i=1,2, \ldots, n
$$

e

$$
f_{i}\left(x_{0}\right)=a_{i}^{T} x_{0}, \quad i=1,2, \ldots, n
$$

no qual $f_{i}: \mathbb{R}^{n} \rightarrow \mathbb{R}$ é o $i$-ésimo componente de $f$.

Expandindo o lado esquerdo de (3.18) em torno de $x_{0}$ e desprezando os termos de ordem superior a dois tem-se:

$$
f_{i}\left(x_{0}\right)+\nabla^{T} f_{i}\left(x_{0}\right)\left(x-x_{0}\right) \approx a_{i}^{T} x,
$$


sendo $\nabla f_{i}(x)=\left[\partial f_{i}(x) / \partial x_{1} \cdots \partial f_{i}(x) / \partial x_{n}\right]^{T}$ o vetor gradiente de $f_{i}(x)$.

Utilizando (3.19), representa-se (3.20) como

$$
\nabla^{T} f_{i}\left(x_{0}\right)\left(x-x_{0}\right) \approx a_{i}^{T}\left(x-x_{0}\right)
$$

onde $x$ é arbitrariamente próximo de $x_{0}$.

O objetivo é encontrar $a_{i}$ que seja o mais próximo possível de $\nabla^{T} f_{i}\left(x_{0}\right)$ e satisfaça $a_{i}^{T} x_{0}=\nabla^{T} f_{i}\left(x_{0}\right)$. Seja

$$
E=\frac{1}{2}\left\|\nabla^{T} f_{i}\left(x_{0}\right)-a_{i}^{T}\right\|
$$

Para satisfazer este objetivo basta resolver o seguinte problema de otimização convexa

$$
\left\{\begin{array}{l}
\operatorname{minimizar}_{a_{i}} E \\
\text { sujeito a } a_{i}^{T} x_{0}=f_{i}\left(x_{0}\right) .
\end{array}\right.
$$

As condições de primeira ordem para o problema de otimização (3.22) são (Chong e Zak, 1996):

$$
\begin{aligned}
& \nabla_{a_{i}} E+\lambda \nabla_{a_{i}}\left(a_{i}^{T} x_{0}-f_{i}\left(x_{0}\right)\right)=0, \\
& a_{i}^{T} x_{0}=f_{i}\left(x_{0}\right) .
\end{aligned}
$$

Manipulando as equações acima é possível chegar na seguinte expressão:

$$
a_{i}=\nabla f_{i}\left(x_{0}\right)+\frac{f_{i}\left(x_{0}\right)-x_{0}^{T} \nabla f_{i}\left(x_{0}\right)}{\left|x_{0}\right|^{2}} x_{0}, \quad x_{0} \neq 0 .
$$

Portanto, é possível encontrar, para qualquer $x_{i} \neq 0$, um modelo linear $A_{i} x+$ $B_{i} u$ a partir de seu modelo verdadeiro (3.5). Através dos $r$ modelos lineares obtidos é possível chegar ao modelo fuzzy T-S desejado.

A escolha da função de pertinência tem influência significativa na precisão do modelo fuzzy. Esta escolha pode ser feita utilizando a experiência e conhecimento do sistema real ou diversos outros métodos envolvendo desde algoritmos de inteligência artificial a métodos estatísticos e de otimização. As funções de pertinência mais comuns na prática são as triangulares e as trapezoidais, pois podem ser implementadas com facilidade. Em casos onde é desejável que as funções 
de pertinência tenham transições mais suaves, pode-se usar funções gaussianas, sigmoidais, dentre outras, desde que estejam definidas no intervalo [ll $\left.\begin{array}{ll}0 & 1\end{array}\right]$.

A aproximação do modelo verdadeiro (3.5) pelo modelo fuzzy (3.2) pode ser obtida de forma mais precisa se as funções de ponderação $\mu_{i}(z(t)), i=1, \ldots, r$, forem determinadas de modo a minimizar o erro de modelagem. Em Teixeira et al. (2002) é apresentado um método para encontrar estas funções de ponderação. A especificação de $\mu_{i}(z(t))$ é dada solucionando um problema de otimização, cujo objetivo é minimizar a norma Euclidiana entre o modelo verdadeiro e o modelo fuzzy.

Segue um exemplo de modelagem fuzzy de um sistema de primeira ordem tratando os pontos abordados.

Exemplo 3.2 (Modelagem de sistemas fuzzy T-S) Considere a função nãolinear $\dot{x}=f(x)$ com

$$
f(x)=\log (x+1)
$$

Deseja-se encontrar um modelo fuzzy T-S que seja uma boa aproximação de (3.24) no intervalo $x \in\left[\begin{array}{ll}0 & 10\end{array}\right]$. O primeiro passo é encontrar os modelos locais do modelo T-S. Na origem é possível encontrar um modelo linear pelo método de linearização tradicional. O modelo na origem é

$$
f_{0}(x)=a_{0} x=x
$$

com $a_{0}$ calculado como em (3.8).

O segundo modelo local será em torno de $x=10$. Observe que este ponto não é um ponto de equilíbrio e que também não é possivel achar um modelo linear utilizando a linearização de Taylor. Será considerado as seguintes aproximações:

- Aproximação afim. O sistema local é a linearização exata em $x=10$, ou seja

$$
f_{1}(x)=f(10)+a_{1}(x-10)=a_{1} x+\left(f(10)-10 a_{1}\right)=a_{1} x+d_{1},
$$

com $a_{1}$ igual a (3.8). Calculando, tem-se $a_{1}=0.0909$ e $d_{1}=1.49$. 
- Aproximação por um modelo linear. $O$ escalar $a_{2}$ é calculado utilizando (3.23). Tem-se

$$
f_{2}(x)=a_{2} x=0.240 x \text {. }
$$

O modelo não-linear, o modelo local na origem e as duas aproximações em $x=10$ podem ser vistas na Figura 3.9. Observe que nos pontos de linearização os modelos são exatos porém, em $x=10$ o modelo afim se aproxima melhor na vizinhança do que o respectivo modelo linear.

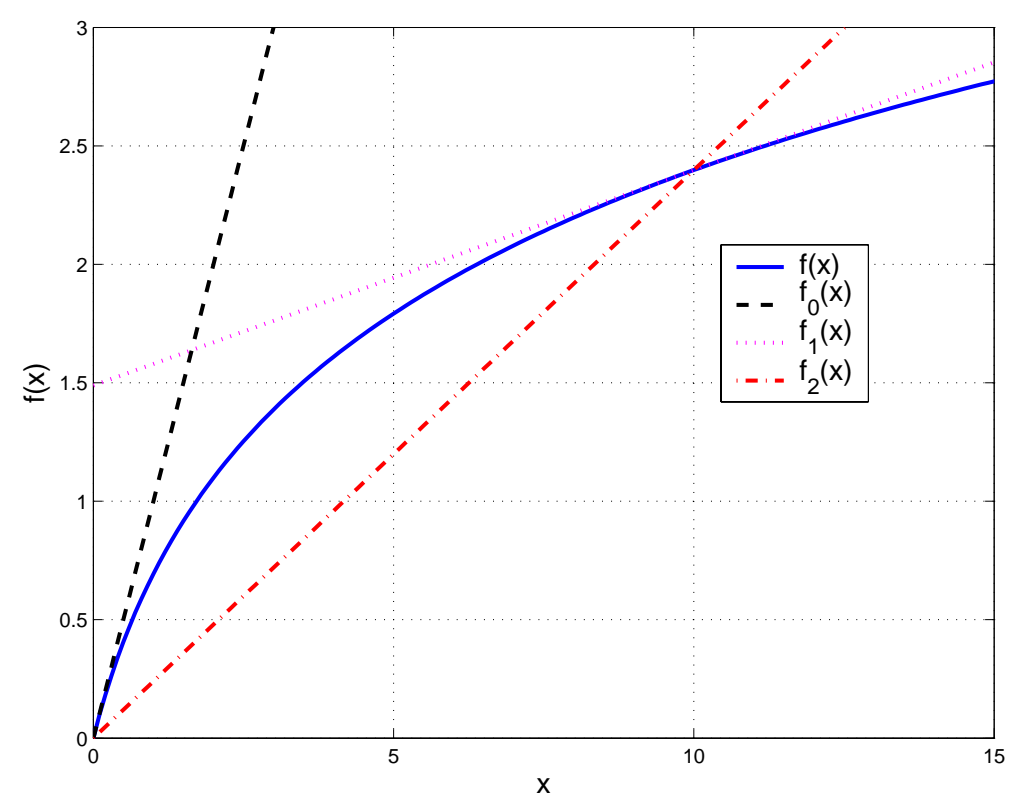

Figura 3.9: Modelo não-linear e modelos locais.

Para obter o modelo global fuzzy utiliza-se as funçôes de pertinência mostradas na Figura 3.10.

Utilizando o modelo linear $f_{2}(x)$ em $x=10$, o modelo global fuzzy é dado por

$$
\dot{x}=f_{f}(x)=\sum_{i=1}^{2} \alpha_{i} f_{i}(x)=\alpha_{1} a_{0} x+\alpha_{2} a_{2} x=\left(\alpha_{1} a_{0}+\alpha_{2} a_{2}\right) x=a_{f} x,
$$

e é ilustrado na Figura 3.11.

Observe que o modelo global não é uma boa aproximação. Modificando o modelo local em $x \approx 10$ para o modelo afim $f_{1}(x)$ tem-se o modelo global mostrado na Figura 3.12. 


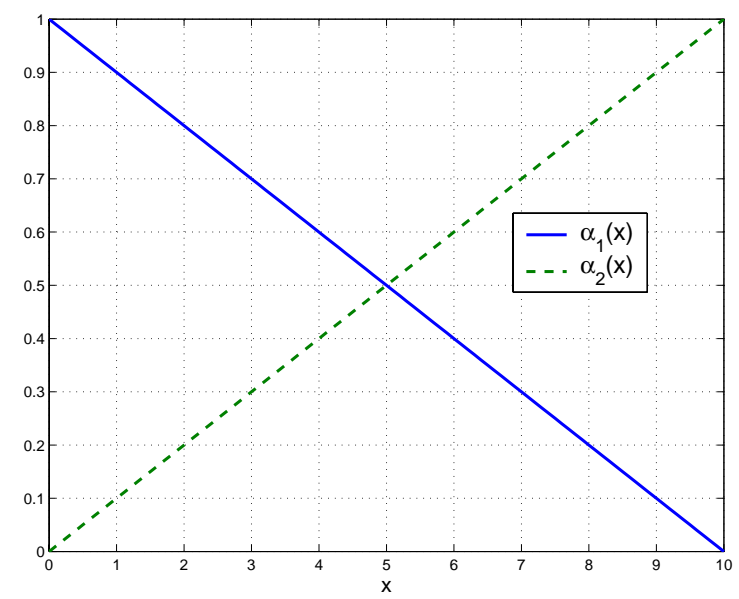

Figura 3.10: Funções de pertinência fuzzy.

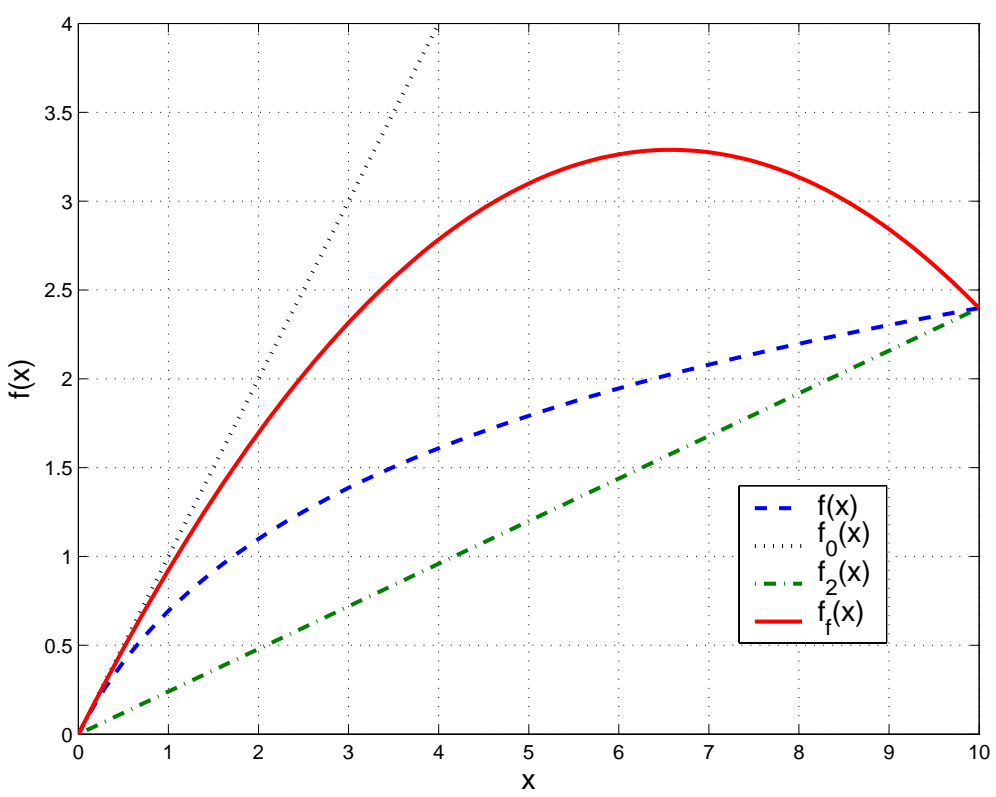

Figura 3.11: Modelo global fuzzy utilizando ambos os modelos lineares.

Apesar do modelo afim ser mais preciso do que o modelo linear na vizinhança do ponto de linearização, este nem sempre proporciona um modelo global mais preciso, como é mostrado nas Figuras 3.11-3.12. Para resolver este problema utiliza-se um número maior de modelos locais de forma a obter um modelo global fuzzy mais próximo do sistema não-linear. Através da aproximação de Teixeira e Zak (1999), utilizando (3.23), obtém-se 10 modelos lineares locais nos pontos $x=1,2,3,4,5,6,7,8,9,10$, além do modelo na origem. Para implementar es- 


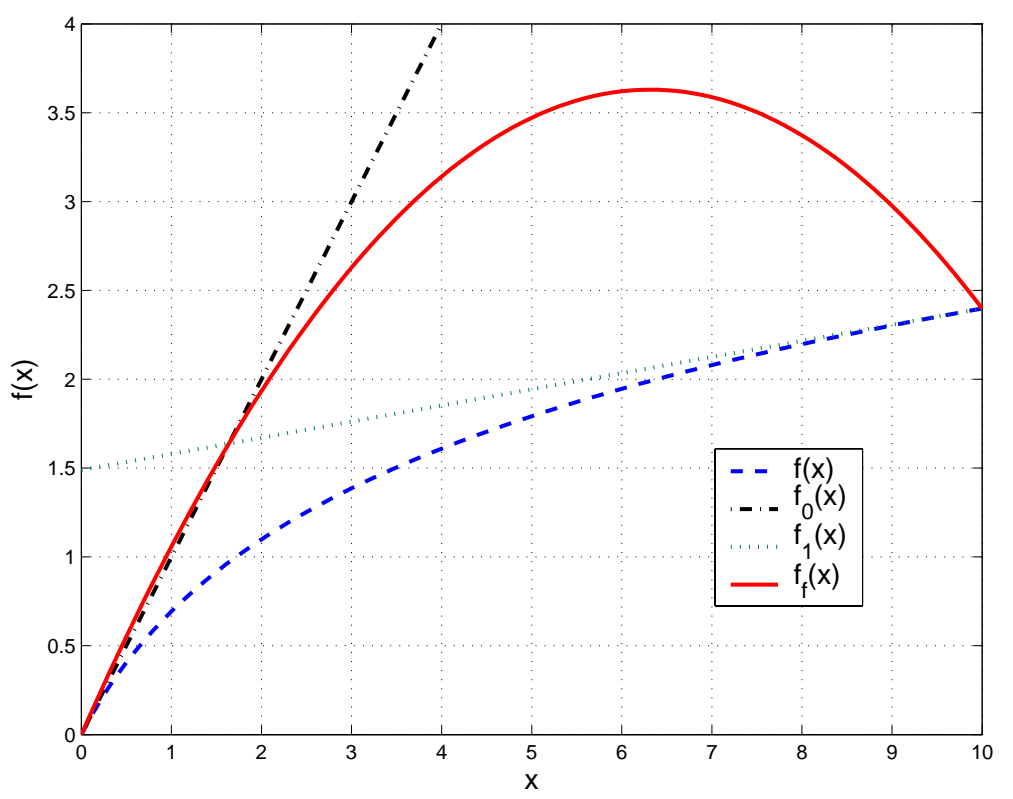

Figura 3.12: Modelo global fuzzy utilizando modelo local afim.

tes modelos locais no modelo global fuzzy escolhe-se as funções de pertinência ilustradas na Figura 3.13.

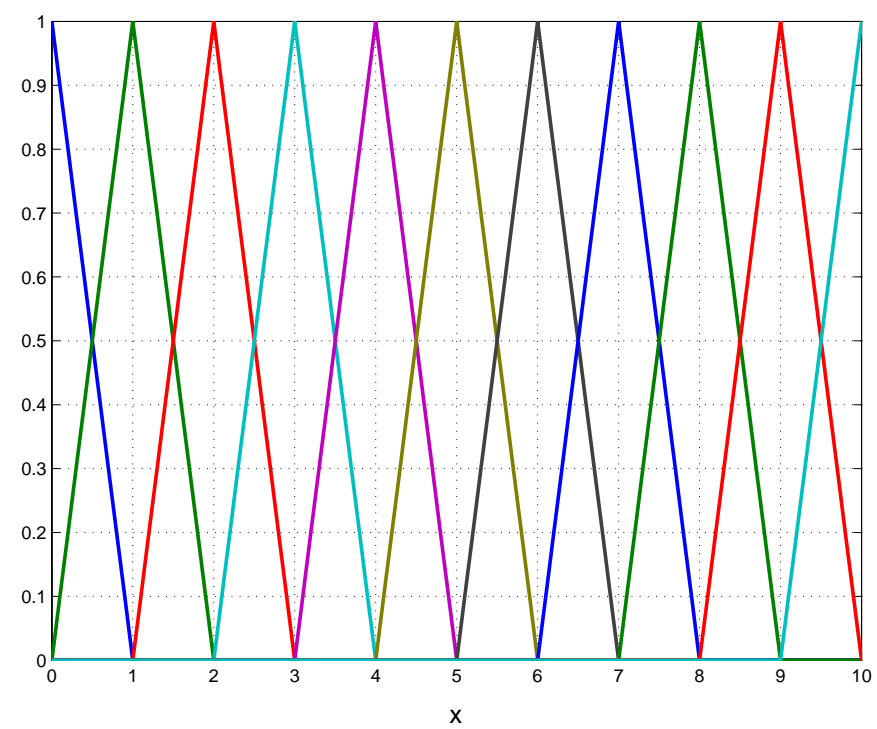

Figura 3.13: Funções pertinências dos 11 modelos lineares locais.

O modelo global fuzzy é ilustrado na Figura 3.14. Com o aumento no número de modelos a precisão do modelo resultante melhora significamente.

Outra alternativa para aumentar a precisão do modelo global fuzzy T-S sem 


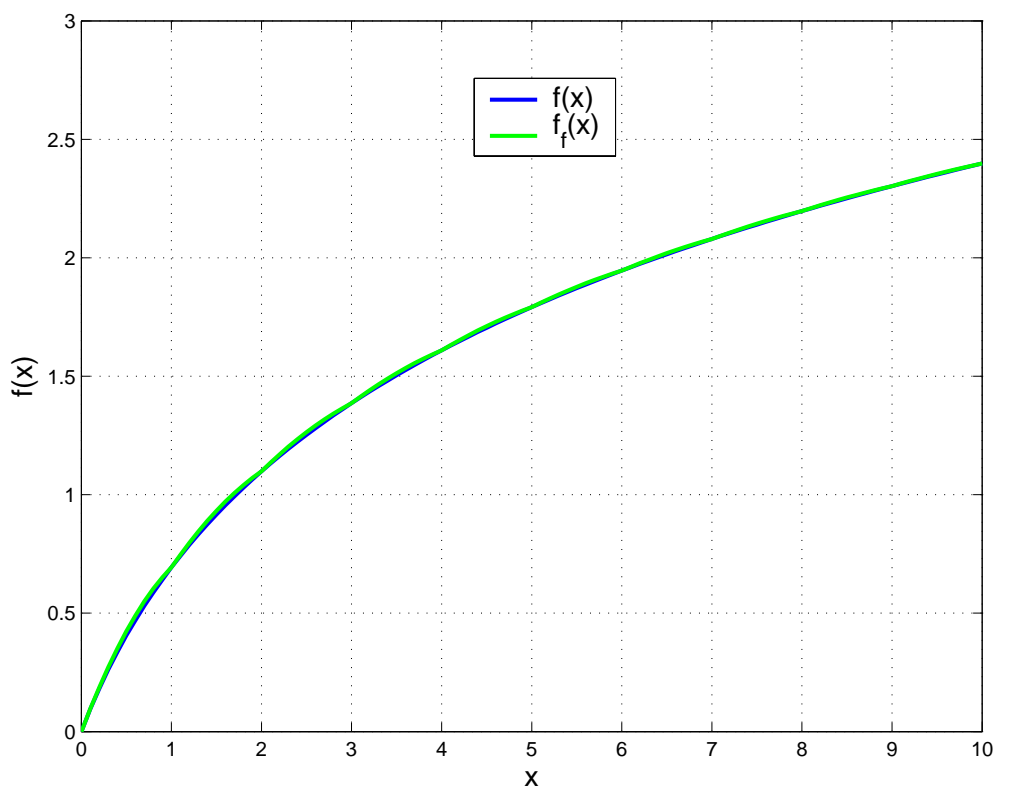

Figura 3.14: Modelo global fuzzy com o uso de 11 modelos lineares locais.

aumentar o número de modelos, o que implica um aumento do esforço computacional e na complexidade nos problemas de análise e síntese, é modificar a função de pertinência adequando-a à característica da planta original. Considere a função de pertinência ilustrada na Figura 3.15. Esta função de pertinência proporciona um peso maior ao modelo local do ponto $x=10$.

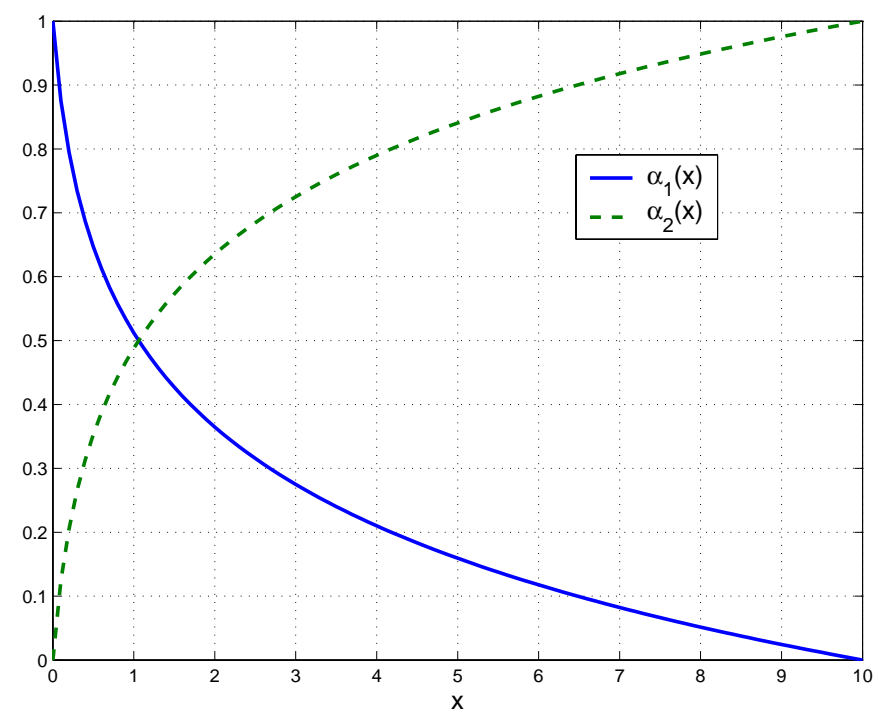

Figura 3.15: Função de pertinência adequada. 


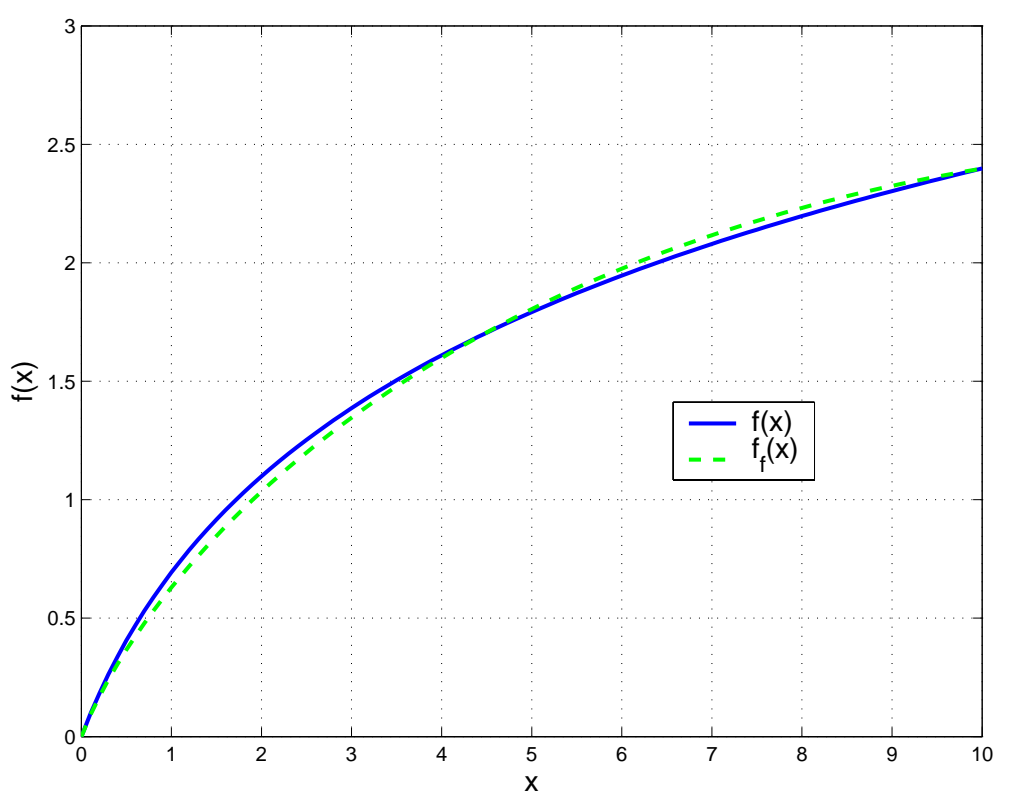

Figura 3.16: Modelo global fuzzy com a função pertinência modificada.

Utilizando o modelo linear $f_{2}(x)$ o modelo global fuzzy resultante pode ser visto na Figura 3.16. Observe que a escolha da função de pertinência tem grande influência na aproximação do sistema não-linear pelo modelo global fuzzy.

\subsection{Controladores Fuzzy Takagi-Sugeno}

\subsubsection{Compensação Distribuída Paralela}

Compensação distribuída paralela (CDP) é o nome dado a um procedimento de projeto de um controlador fuzzy para um dado modelo fuzzy T-S (Wang et al., 1995). Para aplicar o CDP o sistema não-linear deve ser antes representado por um modelo fuzzy T-S.

Em um projeto CDP, cada regra de controle é projetada a partir das regras correspondentes do modelo fuzzy T-S. Para cada regra são utilizadas técnicas de projeto de controle linear. Da regra de modelo fuzzy (3.1) tem-se a seguinte regra para o controlador fuzzy:

\section{Regra de Controle $i$ :}

SE $z_{1}(t)$ é $\mathcal{M}_{i 1}$ e $\cdots$ e $z_{p}(t)$ é $\mathcal{M}_{i p}$ 


$$
\text { ENTÃO } u(t)=K_{i} x(t), \quad i=1,2, \ldots, r .
$$

Os modelo locais fuzzy possuem controladores lineares, como neste exemplo de realimentação de estado. O controlador fuzzy global, que é não-linear em geral, é a combinação dos controladores lineares locais e pode ser representado por:

$$
u(t)=\frac{\sum_{i=1}^{r} w_{i}(z(t)) K_{i} x(t)}{\sum_{i=1}^{r} w_{i}(z(t))}=\sum_{i=1}^{r} \mu_{i}(z(t)) K_{i} x(t) .
$$

O projeto do controlador fuzzy consiste em determinar os ganhos de realimentação locais $K_{i}$. Apesar do controlador fuzzy (3.25) ser construído em função dos ganhos locais, o ganho de realimentação $K_{i}$ deve ser determinado utilizando condições de projeto globais a fim de garantir estabilidade e desempenho globais.

\subsubsection{Condições de Estabilidade}

As condições de suficiência para a estabilidade de sistemas fuzzy derivam da teoria de estabilidade de Lyapunov usando funções de Lyapunov quadráticas do tipo $V(x(t))=x^{T}(t) P x(t)$ (Tanaka e Sugeno, 1992).

Teorema 3.3 O sistema fuzzy contínuo descrito por (3.2) com $u(t)=0$ é globalmente assintoticamente estável se existir uma matriz comum simétrica positiva definida $P$ tal que

$$
A_{i}^{T} P+P A_{i}<0
$$

para $i=1,2, \ldots, r$, isto é, uma $P$ comum a todos os subsistemas.

Prova: Segue diretamente da aplicação da função de Lyapunov

$$
V(x(t))=x^{T}(t) P x(t) .
$$

Substituindo (3.25) em (3.2) podemos escrever a equação em malha fechada como

$$
\dot{x}(t)=\sum_{i=1}^{r} \sum_{j=1}^{r} \mu_{i}(z(t)) \mu_{j}(z(t))\left\{A_{i}+B_{i} K_{j}\right\} x(t) .
$$


Denotando

$$
G_{i j}=A_{i}+B_{i} K_{j}
$$

a equação (3.26) pode ser reescrita como

$$
\dot{x}(t)=\sum_{i=1}^{r} \mu_{i}(z(t)) \mu_{i}(z(t)) G_{i i} x(t)+2 \sum_{i=1}^{r} \sum_{i<j} \mu_{i}(z(t)) \mu_{j}(z(t))\left\{\frac{G_{i j}+G_{j i}}{2}\right\} x(t) .
$$

Aplicando as condições de estabilidade do Teorema 3.3 a (3.27) pode-se obter a seguinte condição de estabilidade em malha fechada.

Teorema 3.4 O sistema fuzzy descrito por (3.27) é globalmente assintoticamente estável se existir uma matriz comum $P$ simétrica positiva definida tal que

$$
\begin{gathered}
G_{i i}^{T} P+P G_{i i}<0, \\
\left(\frac{G_{i j}+G_{j i}}{2}\right) P+P \\
\left(\frac{G_{i j}+G_{j i}}{2}\right) \leq 0, \\
i<j \text { sujeito } a \mu_{i} \bigcap \mu_{j} \neq \emptyset .{ }^{1}
\end{gathered}
$$

Prova: Segue diretamente do Teorema 3.3.

\subsubsection{Projeto de Controladores Estáveis}

O problema de projeto fuzzy é determinar os ganhos de realimentação $K_{j}, j=$ $1,2, \ldots, r$, que satisfaçam as condições do Teorema 3.4 com uma matriz $P$ comum (Tanaka e Wang, 2001). As desigualdades (3.28) e (3.29) não são convexas, para o problema de síntese, e por isso não podem ser resolvidas diretamente através de LMIs. Para ser possível expressar o problema em LMIs é necessário transformá-lo em um problema convexo, para tal, multiplica-se ambos os lados das equações

\footnotetext{
${ }^{1} \mathrm{Na}$ notação utilizada neste trabalho significa que esta condição deve valer para todo $i<j$ exceto quando $\mu_{i} \bigcap \mu_{j}=\emptyset$, ou seja, $\mu_{i}(z(t)) \times \mu_{j}(z(t))=0$ para todo $z(t)$, onde $\mu_{i}(z(t))$ denota o peso da i-ésima regra calculada da função de pertinência na variável premissa. Note que $\mu_{i} \bigcap \mu_{j}=\emptyset$ se, e somente se, a i-ésima regra e a j-ésima regra não se sobrepõe.
} 
por $P^{-1}$ e define-se uma nova variável $X=P^{-1}$, obtendo

$$
\begin{array}{r}
X A_{i}^{T}+A_{i} X+X K_{i}^{T} B_{i}^{T}+B_{i} K_{i} X<0 \\
X A_{i}^{T}+A_{i} X+X A_{j}^{T}+A_{j} X+X K_{j}^{T} B_{i}^{T}+B_{i} K_{j} X+X K_{i}^{T} B_{j}^{T}+B_{j} K_{i} X \leq 0 .
\end{array}
$$

Definindo $Q_{i}=K_{i} X$ tal que para $X>0$ tem-se $K_{i}=Q_{i} X^{-1}$. Substituindo $K_{i}$ nas desigualdades acima tem-se

$$
\begin{array}{r}
X A_{i}^{T}+A_{i} X+Q_{i}^{T} B_{i}^{T}+B_{i} Q_{i}<0 \\
X A_{i}^{T}+A_{i} X+X A_{j}^{T}+A_{j} X+Q_{j}^{T} B_{i}^{T}+B_{i} Q_{j}+Q_{i}^{T} B_{j}^{T}+B_{j} Q_{i} \leq 0
\end{array}
$$

Portanto, o projeto do controlador fuzzy estável se resume a encontrar $X>0$ e $Q_{i}, i=1, \ldots, r$, satisfazendo

$$
\begin{array}{r}
X A_{i}^{T}+A_{i} X+Q_{i}^{T} B_{i}^{T}+B_{i} Q_{i}<0 \\
X A_{i}^{T}+A_{i} X+X A_{j}^{T}+A_{j} X+Q_{j}^{T} B_{i}^{T}+B_{i} Q_{j}+Q_{i}^{T} B_{j}^{T}+B_{j} Q_{i} \leq 0 \\
i<j \text { sujeito a } \mu_{i} \bigcap \mu_{j} \neq \emptyset
\end{array}
$$

no qual

$$
X=P^{-1}, \quad Q_{i}=K_{i} X
$$

As condições acima são LMIs com relação às variáveis $X$ e $Q_{i}$. O ganho de realimentação $K_{i}$ e a matriz $P$ comum podem ser obtidas através de

$$
P=X^{-1}, \quad K_{i}=Q_{i} X^{-1}
$$

Exemplo 3.3 (Estabilização de um pêndulo invertido) Considere o problema de controle de um pêndulo invertido em um carro em movimento, conforme ilustra a Figura 3.17. O movimento do pêndulo pode ser descrito pelas equações

$$
\begin{aligned}
& \dot{x}_{1}=x_{2} \\
& \dot{x}_{2}=\frac{g \operatorname{sen}\left(x_{1}\right)-a m l x_{2}^{2} \operatorname{sen}\left(2 x_{1}\right) / 2-a \cos \left(x_{1}\right) u}{4 l / 3-a m l \cos ^{2}\left(x_{1}\right)},
\end{aligned}
$$

onde $x_{1}$ denota o ângulo do pêndulo com a vertical, $x_{2}$ é a velocidade angular, $g=9,8 \mathrm{~m} / \mathrm{s}^{2}$ é a constante gravitacional, $m$ é a massa do pêndulo, $M$ é a massa do carro, $a=1 /(m+M), 2 l$ é o comprimento do pêndulo e u é a força aplicado 


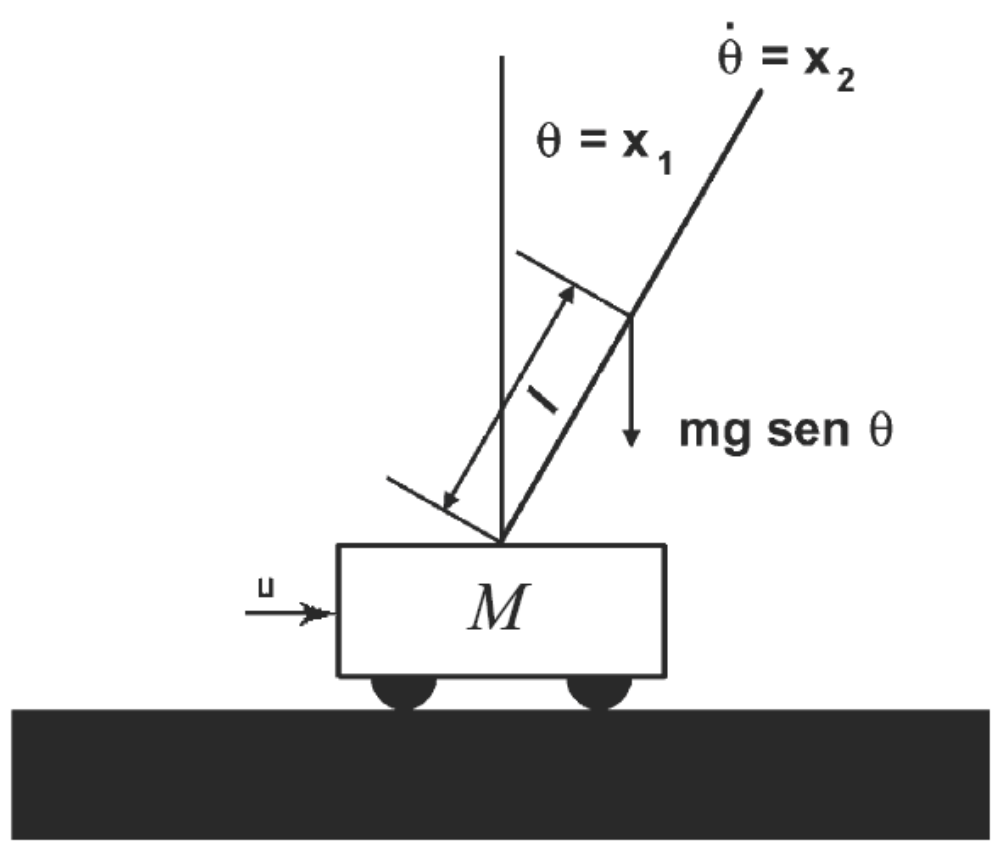

Figura 3.17: Carro com um pêndulo invertido.

ao carro. Os seguintes parâmetros foram escolhidos: $m=2 \mathrm{Kg}, M=8 \mathrm{Kg} e$ $l=0.5 m$.

Para obter o controlador fuzzy é necessário linearizar o sistema original em pontos de operação escolhidos. O objetivo do controle é estabilizar o pêndulo na faixa aproximada $x_{1} \in(-\pi / 2, \pi / 2)$. O sistema (3.31) possui pontos de equilibrio em $x_{1}= \pm k \pi, k=0,1,2, \ldots$, e $x_{2}=0$. Portanto, escolheu-se os pontos $(0,0) e$ $(\approx \pm \pi / 2,0)$ para a linearização. Através da técnica de linearização convencional (3.7) obtém-se o sistema linear na origem.

Seja $\dot{x}=F(x, u)$.

$$
\begin{aligned}
A\left(x_{0}, u_{0}\right) & =\frac{\partial F}{\partial x}\left(x_{0}, u_{0}\right) \\
& =\left[\begin{array}{ll}
\frac{\partial F_{1}}{\partial x_{1}}(x, u) & \frac{\partial F_{1}}{\partial x_{2}}(x, u) \\
\frac{\partial F_{2}}{\partial x_{1}}(x, u) & \frac{\partial F_{2}}{\partial x_{2}}(x, u)
\end{array}\right]_{\left(x_{0}, u_{0}\right)},
\end{aligned}
$$


com

$$
\begin{aligned}
\frac{\partial F_{1}}{\partial x_{1}}(x, u)= & 0 \\
\frac{\partial F_{1}}{\partial x_{2}}(x, u)= & 1 \\
\frac{\partial F_{2}}{\partial x_{1}}(x, u)= & \frac{\left(4 l / 3-a m l \cos ^{2}\left(x_{1}\right)\right)\left(g \cos \left(x_{1}\right)-a m l x_{2}^{2} \cos \left(x_{1}\right)+a \operatorname{sen}\left(x_{1}\right) u\right)}{\left(4 l / 3-a m l \cos ^{2}\left(x_{1}\right)\right)^{2}} \\
& +\frac{\left(2 \operatorname{sen}\left(x_{1}\right) \cos \left(x_{1}\right)\right)\left(g \operatorname{sen}\left(x_{1}\right)-a m l x_{2}^{2} \operatorname{sen}\left(2 x_{1}\right) / 2-a \cos \left(x_{1}\right) u\right)}{\left(4 l / 3-a m l \cos ^{2}\left(x_{1}\right)\right)^{2}}, \\
\frac{\partial F_{2}}{\partial x_{2}}(x, u)= & -a m l x_{2} \operatorname{sen}\left(2 x_{1}\right)
\end{aligned}
$$

e

$$
B\left(x_{0}, u_{0}\right)=\frac{\partial F}{\partial u}\left(x_{0}, u_{0}\right)=\left[\begin{array}{c}
0 \\
\frac{-a \cos \left(x_{1}\right)}{4 l / 3-a m l \cos ^{2}\left(x_{1}\right)}
\end{array}\right]_{\left(x_{0}, u_{0}\right)}
$$

Na origem tem-se:

$$
\begin{aligned}
& A(0,0,0)=\left[\begin{array}{cc}
0 & 1 \\
\frac{g}{4 l / 3-a m l} & 0
\end{array}\right], \\
& B(0,0,0)=\left[\begin{array}{c}
0 \\
\frac{-a}{4 l / 3-a m l}
\end{array}\right] .
\end{aligned}
$$

Observe que o mesmo procedimento efetuado no ponto $(\approx \pm \pi / 2,0)$ resulta em um sistema afim, ao invés de linear, devido a presença dos termos $F\left(x_{0}, u_{0}\right)$ $e-\left(A x_{0}+B u_{0}\right)$. Para contornar este problema será utilizado a metodologia proposta na Seção 3.1.2 (Teixeira e Zak, 1999).

Aplicando (3.23) para $x_{1}=88^{\circ} \pi / 180^{\circ}=1,536 \mathrm{rad}$ e $x_{2}=0$, tem-se

$$
\begin{aligned}
& a_{1}^{T}=\left[\begin{array}{ll}
0 & 1
\end{array}\right], \\
& a_{2}^{T}=\left[\begin{array}{ll}
\frac{2 g}{\pi(4 l / 3-a m l \phi)} & 0
\end{array}\right],
\end{aligned}
$$

onde $\phi=\cos \left(88^{\circ}\right)$ e $a_{i}, i=1,2$, é a $i$-ésima linha de A. Portanto,

$$
A\left(88^{\circ} \pi / 180^{\circ}, 0,0\right)=\left[\begin{array}{cc}
0 & 1 \\
\frac{2 g}{\pi(4 l / 3-a m l \phi)} & 0
\end{array}\right] .
$$


A matriz $B$ é obtida a partir de (3.32),

$$
B\left(88^{\circ} \pi / 180^{\circ}, 0,0\right)=\left[\begin{array}{c}
0 \\
-a \phi \\
4 l / 3-a m l \phi^{2}
\end{array}\right] .
$$

Substituindo os valores dos parâmetros tem-se

$$
\begin{aligned}
& A_{1}=A(0,0,0)=\left[\begin{array}{cc}
0 & 1 \\
16.18 & 0
\end{array}\right], \quad B_{1}=B(0,0,0)=\left[\begin{array}{c}
0 \\
-0.081
\end{array}\right], \\
& A_{2}=A\left(88^{\circ} \pi / 180^{\circ}, 0,0\right)=\left[\begin{array}{ll}
0 & 1 \\
4.24 & 0
\end{array}\right], \quad B_{2}=B\left(88^{\circ} \pi / 180^{\circ}, 0,0\right)=\left[\begin{array}{c}
0 \\
-0.0026
\end{array}\right] .
\end{aligned}
$$

Através da análise dos autovalores verifica-se que a origem é um ponto de equilíbrio instável. A partir dos sistemas linearizados acima as seguintes regras fuzzy são utilizadas para descrever o sistema:

\section{Regra de Modelo 1:}

$S E$

$$
x_{1}(t) \text { está em torno } 0 \text {, }
$$

ENTẼO $\quad \dot{x}(t)=A_{1} x(t)+B_{1} u(t)$.

\section{Regra de Modelo 2:}

$S E$

$x_{1}(t)$ está em torno $\pm \pi / 2$,

ENTĨO $\quad \dot{x}(t)=A_{2} x(t)+B_{2} u(t)$.

São usadas as seguintes funções de pertinência

$$
\begin{aligned}
& \mu_{1}\left(x_{1}\right)=\frac{1-1 /\left(1+\exp \left(-7\left(x_{1}-\pi / 4\right)\right)\right)}{1+\exp \left(-7\left(x_{1}+\pi / 4\right)\right)} \\
& \mu_{2}\left(x_{1}\right)=1-\mu_{1}\left(x_{1}\right) .
\end{aligned}
$$

Foi obtido, através da resolução das LMIs (3.30), no software Matlab, os seguintes controladores fuzzy via CDP

$$
\begin{aligned}
& K_{1}=\left[\begin{array}{ll}
1557.5 & 662.5
\end{array}\right] \\
& K_{2}=\left[\begin{array}{ll}
3339.1 & 1496.3
\end{array}\right]
\end{aligned}
$$




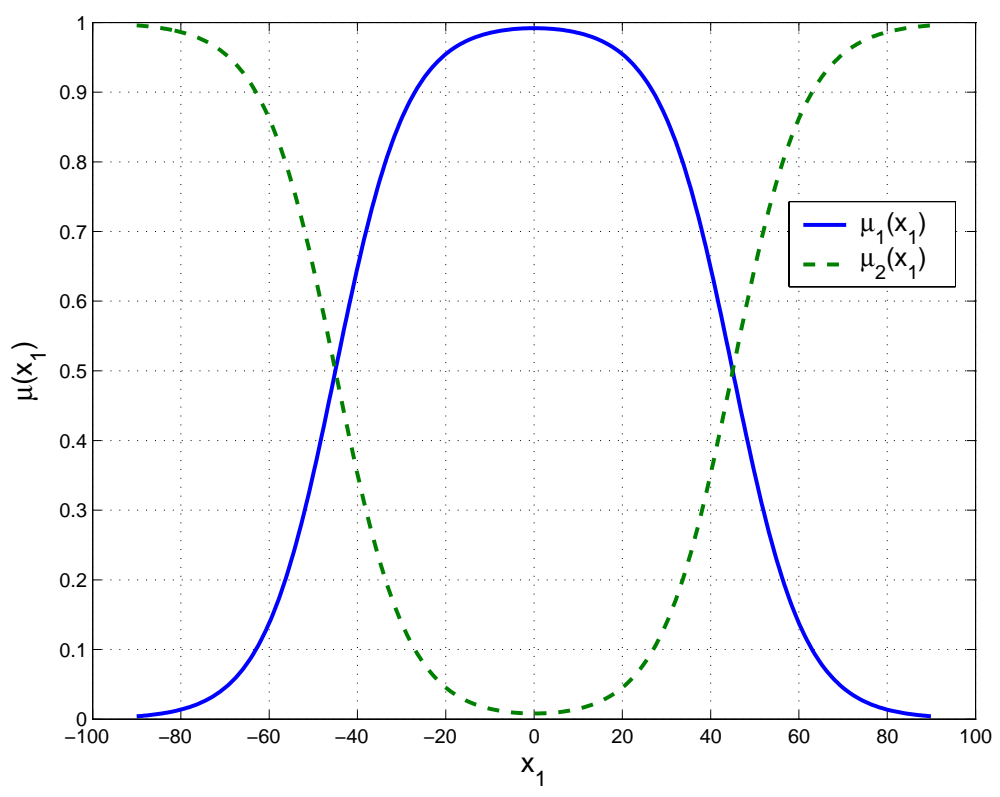

Figura 3.18: Funções de pertinência fuzzy.

com

$$
\begin{aligned}
P & =\left[\begin{array}{cc}
75.6 & -149.1 \\
-149.1 & 311.82
\end{array}\right], \\
Q_{1} & =\left[\begin{array}{ll}
18881.0 & -25712.3
\end{array}\right], \\
Q_{2} & =\left[\begin{array}{ll}
29137.0 & -31410.6
\end{array}\right] .
\end{aligned}
$$

A implementação do controlador fuzzy foi realizada no módulo Simulink do Matlab utilizando as equações diferenciais do pêndulo (3.31), conforme ilustra a Figura 3.19. As Figuras 3.20 e 3.21 ilustram a implementação do controle fuzzy em malha fechada.

Simulações indicam que o controlador é capaz de estabilizar o pêndulo para condições iniciais $x_{10} \in\left[\begin{array}{ll}-87.5^{0} & 87.5^{0}\end{array}\right]\left(x_{20}=0\right)$. Em contraste, o controlador linear $u=K_{1} x$, da Figura 3.22, falha quando $\left|x_{10}\right|>85^{\circ}$. A Figura 3.23 ilustra a resposta angular $x_{1}(t)$ para as condições iniciais $x_{10}=87^{0}, 85^{0}, 75^{0}, 45^{0}$ e $20^{0}$ e $x_{20}=0$ para os controladores linear e fuzzy. Observe que o controlador fuzzy consegue melhores respostas para ângulos maiores, visto que o projeto leva em consideração a linearização em torno de $x_{1} \approx \pm \pi / 2$.

Também é possível desenvolver uma lei de controle não-linear para a planta 


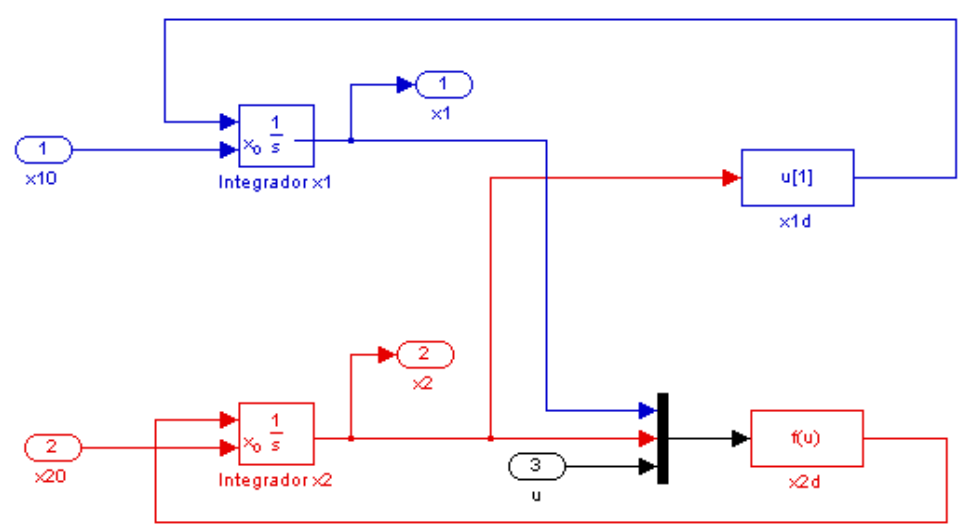

Figura 3.19: Modelo do pêndulo invertido no Simulink.

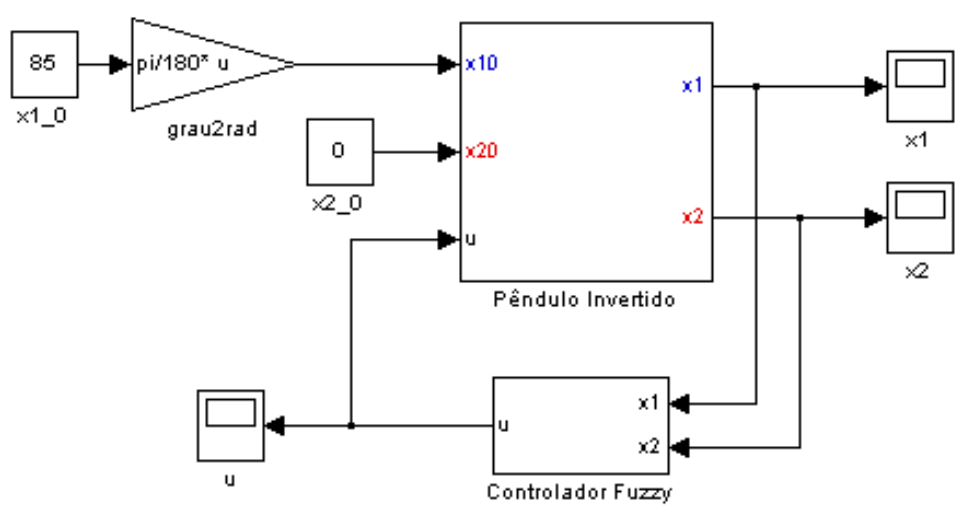

Figura 3.20: Sistema em malha fechada com o controlador fuzzy.

(3.31) e ângulos iniciais $x_{10} \in(-\pi / 2, \pi / 2)$ (Baumann e Rugh, 1986), contudo tal projeto tende a ser mais complexo. Em contraste, o projeto CDP é intuitivo e simples assim como o controlador encontrado. 


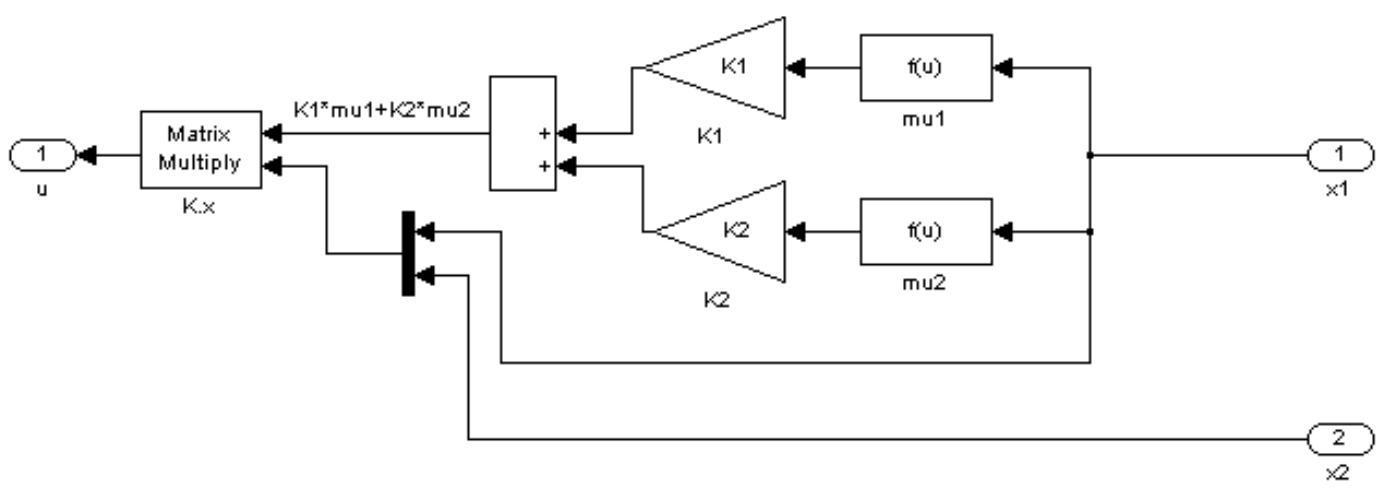

Figura 3.21: Estrutura do controlador fuzzy via CDP.

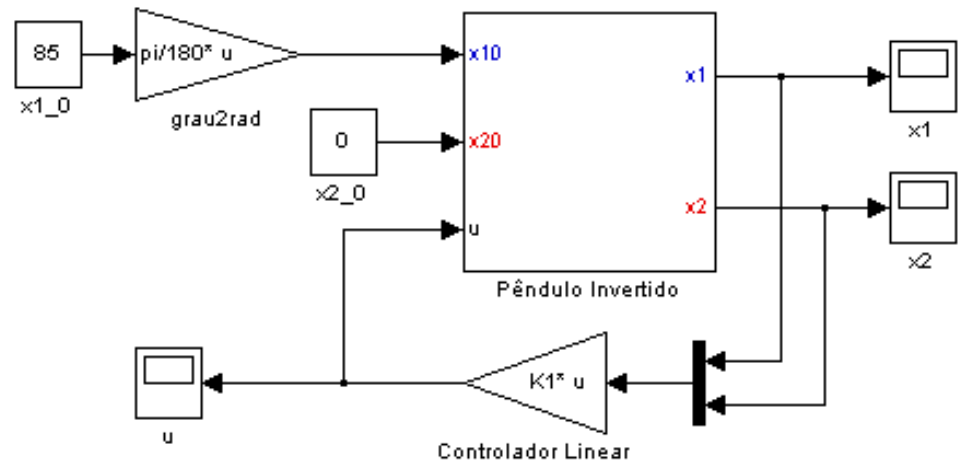

Figura 3.22: Controlador linear. 


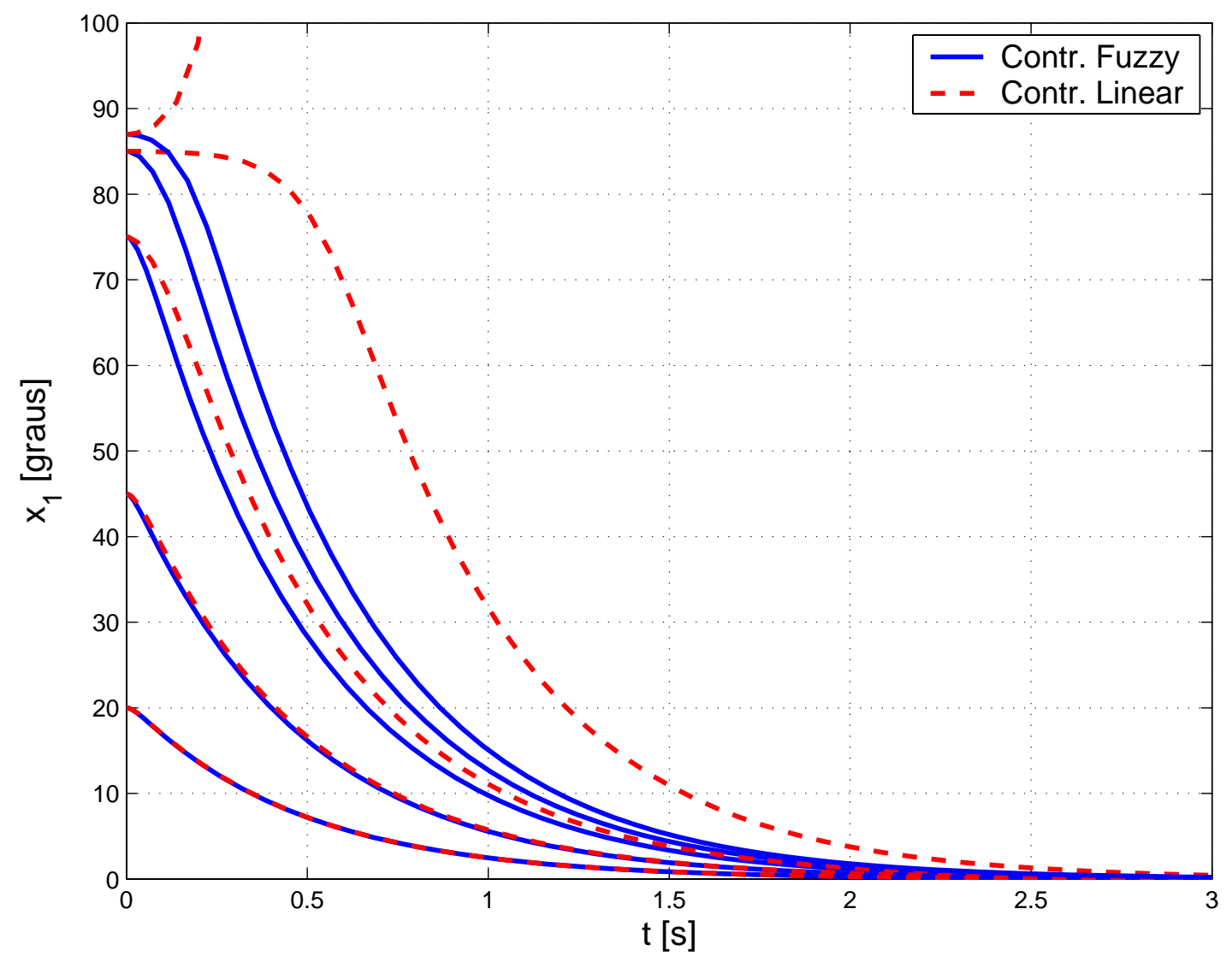

Figura 3.23: Resposta do ângulo do pêndulo para diversas condições iniciais com o controlador fuzzy CDP (linha contínua) e o controlador linear clássico (linha tracejada). 


\section{Capítulo 4}

\section{Controle Linear por Partes}

\subsection{Introdução}

Ao aplicar técnicas de controle conhecidas geralmente opta-se por linearizar o modelo em torno de um ponto de operação para que as técnicas lineares posam ser mais facilmente aplicadas. A utilização de técnicas de controle linear por partes tem aumentado no estudo de sistemas não-lineares.

Na engenharia muitos sistemas são descritos sob a ótica de sistemas lineares por partes como exemplo relês, diodos e transistores. A construção de um modelo global não-linear a partir de linearizações locais é um conceito fácil de entender e aplicável em diversas situações.

Serão apresentadas neste capítulo as técnicas de controle linear por partes, construção de um modelo dividindo o espaço de estado em partições, análises de estabilidade e aplicações em sistemas fuzzy, que interessará ao desenvolvimento da técnica proposta neste trabalho.

Neste trabalho, por termo linear por partes entende-se um sistema que apresente diferentes dinâmicas lineares em diferentes regiões no espaço de estado. 


\subsection{Modelagem Linear por Partes}

Considere o sistema dinâmico

$$
\left\{\begin{array}{l}
\dot{x}=f(x, u, t) \\
y=g(x, u, t) .
\end{array}\right.
$$

o qual o lado direito da equação é uma função linear por partes de seus argumentos. Por exemplo, uma função que a entrada satura resulta num sistema que é linear por partes na variável de entrada. Sistemas lineares com mudanças abruptas em seus parâmetros são sistemas lineares por partes no tempo. A situação mais comum, contudo, é quando as equações de sistema são lineares por partes no estado. Esta situação ocorre por exemplo em linearizações de sistemas não-lineares em torno de diferentes pontos de operação ou da interconexão de componentes lineares por partes com sistemas não-lineares.

É possível subdividir o espaço de estado em conjuntos de regiões $\mathcal{X}_{i} \subset \mathbb{R}^{n}$ tais que a dinâmica dentro de cada região seja linear, dada por

$$
\left\{\begin{array}{l}
\dot{x}(t)=A_{i} x(t)+B_{i} u(t) \\
y(t)=C_{i} x(t)+D_{i} u(t)
\end{array} \quad \text { para } x(t) \in \mathcal{X}_{i},\right.
$$

onde $i$ é o índice do subespaço, $x(t) \in \mathbb{R}^{n}$ o estado do sistema, $u(t) \in \mathbb{R}^{p}$ a variável de entrada e $y(t)$ a de saída.

É possível dividir o espaço de estado em regiões de qualquer geometria porém, neste trabalho, estar-se-á restringindo o particionamento do espaço de estados em poliedros convexos a fim de diminuir a complexidade na busca de funções de descrevam estas partições. Esta geometria se justifica na aplicação em sistemas fuzzy, visto que as partições são determinadas pelas funções de pertinência das variáveis premissas.

Ao se representar um sistema linear por partes é possível que termos afins apareçam. Isto pode acontecer na linearização de sistemas não-lineares em tornos de pontos de operação ou na representação de saturações, como mostra o exemplo a seguir. 
Exemplo 4.1 (Atuador saturado) Considere o sistema linear abaixo sob realimentação de estado saturado pelo atuador,

$$
\dot{x}=A x+b \operatorname{sat}(v), \quad v=k^{T} x .
$$

A saturação induz a uma divisão poliedral natural do espaço de estado em três regiões, conforme a Figura 4.1. A dinâmica é linear por partes e descrita por

$$
\dot{x}=\left\{\begin{array}{ll}
A x-b & x \in X_{1} \\
\left(A+b k^{T}\right) x & x \in X_{2} \\
A x+b & x \in X_{3}
\end{array} .\right.
$$
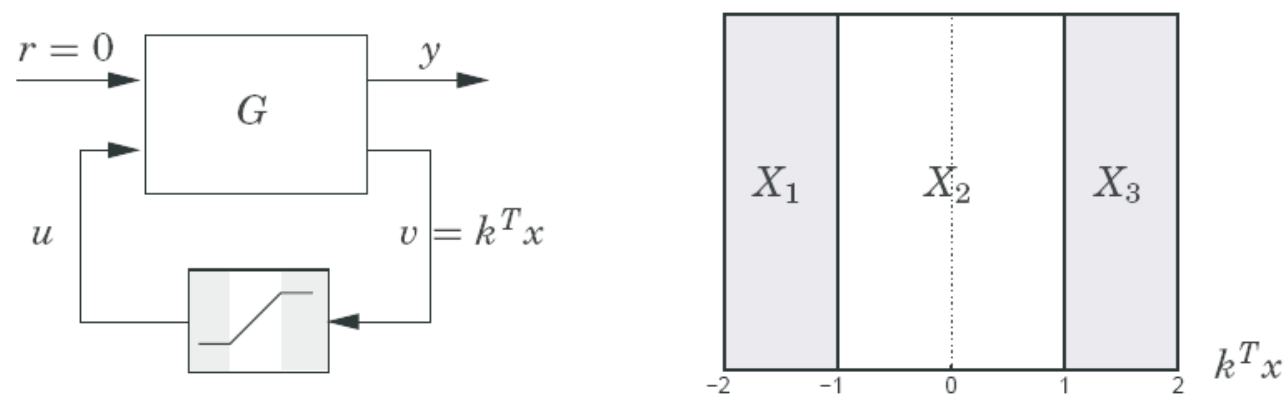

Figura 4.1: Uma realimentação linear saturada (esquerda) leva a um sistema linear por partes com uma divisão poliedral do espaço de estado (direita).

A presença de um termo constante faz com que a dinâmica seja afim ao invés de linear no estado $x$.

Neste trabalho dá-se ênfase à análise de sistemas lineares ao invés de afins visto que a presença de termos afins em linearizações de sistemas não-lineares pode ser evitada, como visto na seção 3.1.2. Utilizando somente sistemas lineares na representação das dinâmicas nas partições ganha-se na menor complexidade das técnicas de controle, porém, há a necessidade de um número maior de modelos para representar o sistema não-linear. 


\subsection{Estabilidade de Sistemas Lineares por Par- tes}

Considera-se o sistema linear por partes da seguinte forma

$$
\dot{x}(t)=A_{i} x(t)+B_{i} u(t), \quad \text { para } x(t) \in \mathcal{X}_{i},
$$

onde $\mathcal{X}_{i}$ é a partição no espaço de estado dos regimes de operação.

A estabilidade de um sistema pode ser definida de várias formas. Como já se viu anteriormente a estabilidade pode ser analisada no senso de Lyapunov, através de uma função energia $V(x)$.

Considere o seguinte resultado.

Lema 4.1 (Johansson, 1999) Seja $x(t):[0, \infty) \rightarrow \mathbb{R}^{n}$ e seja $V(t):[0, \infty) \rightarrow \mathbb{R}$ uma função não crescente e por partes $\mathcal{C}^{1}$ satisfazendo

$$
\dot{V}(t) \leq-\gamma\|x(t)\|^{p}
$$

para algum $\gamma>0$ e algum $p>0$, em quase todo $[0, \infty)$. Se existir $\alpha>0$ e $\beta>0$ finito tal que

$$
\alpha\|x(t)\|^{p} \leq V(t) \leq \beta\|x(t)\|^{p}
$$

então $\|x(t)\|$ tende a zero exponencialmente.

Prova: Vide Johansson (1999).

Esta formulação não requer que $V(t)$ seja contínua contanto que o valor de $V(t)$ não aumente nos pontos de descontinuidade.

Analogamente ao caso geral, é possível estabelecer a estabilidade de sistemas lineares por partes através de uma matriz $P$ que leve a uma função $V(x)$ que satisfaça as condições do Lema 4.1.

Proposição 4.2 (Johansson, 1999) Considere o sistema (4.2) com u(t) $\equiv 0$. Se o problema de otimização convexa

$$
\begin{aligned}
& P=P^{T}>0, \\
& A_{i}^{T} P+P A_{i}<0, \quad i \in I
\end{aligned}
$$


tem solução, com I o conjunto de índices de todas as regiões $\mathcal{X}_{i}$ do espaço de estado, então toda trajetória de $x(t) \in \cup_{i \in I} \mathcal{X}_{i}$ de (4.2) tende a zero exponencialmente.

Prova: Vide Johansson (1999).

\section{Exemplo 4.2 (Ilustração do uso de funções de Lyapunov contínuas} por partes) Considere a seguinte equação diferencial não-linear (Johansson e Rantzer, 1998):

$$
\dot{x}= \begin{cases}A_{1} x, & \text { se } x_{1} \leq 0 \\ A_{2} x, & \text { se } x_{1} \geq 0\end{cases}
$$

onde

$$
A_{1}=\left[\begin{array}{ll}
-5 & -4 \\
-1 & -2
\end{array}\right] \text { e } A_{2}=\left[\begin{array}{cc}
-2 & -4 \\
20 & -2
\end{array}\right]
$$

Resolvendo (4.4) não é possível encontrar uma função de Lyapunov quadrática global $V(x)=x^{T} P x$ que assegure a estabilidade do sistema. Porém, as simulações mostradas na Figura 4.2 indicam que o sistema é estável. Para provar a estabilidade faz-se necessário, portanto, uma nova abordagem. O uso de funções de Lyapunov contínua por partes pode auxiliar a resolução do problema de análise de estabilidade.

Uma extensão natural para sistemas lineares por partes é considerar funções de Lyapunov contínuas que são quadráticas por partes,

$$
V(x)=x^{T} P_{i} x, \quad \text { para } x \in \mathcal{X}_{i}
$$

Estas funções possuem diferentes comportamentos em diversos regimes de operação e são mais poderosas do que as funções quadráticas globais. Um problema fundamental na busca de funções de Lyapunov por partes é como a restrição de continuidade deve ser considerada na busca de uma função candidata.

Seja $\partial \mathcal{X}_{i}$ a fronteira do subespaço $\mathcal{X}_{i}$, ou seja, $\partial \mathcal{X}_{i}=\left\{x(t): x(t) \in \mathcal{X}_{i} \cap \mathcal{X}_{j}\right\}$ Para obter continuidade através da fronteira deve-se ter

$$
V_{i}(x)=V_{j}(x), \quad \forall x \in \partial \mathcal{X}
$$




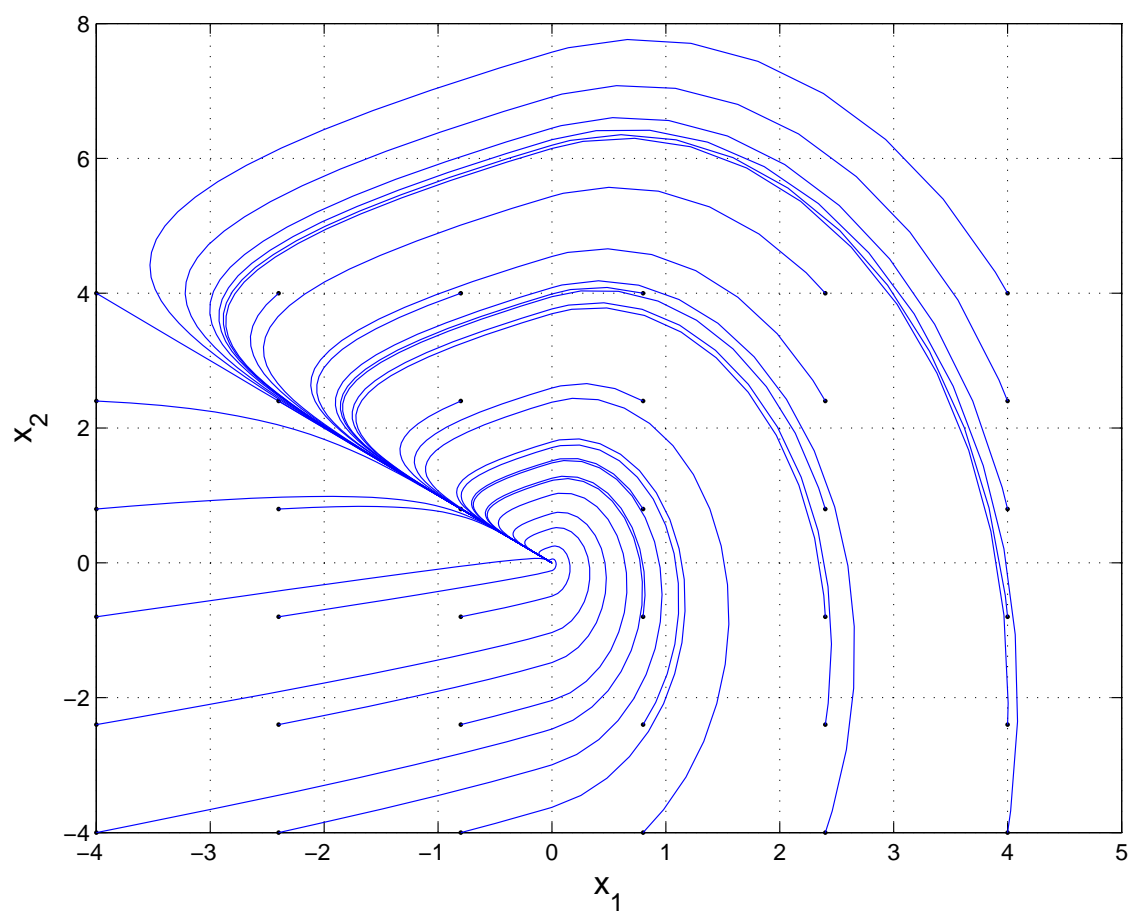

Figura 4.2: Trajetórias do sistema. Os pontos em preto significam a condição inicial da trajetória.

Porém, é difícil encontrar uma expressão que se adeque como restrição no problema de otimização convexa. Como solução tem-se uma parametrização da função quadrática por partes através do uso de uma matriz de continuidade.

Definição 4.3 (Matriz de continuidade) Uma matriz $F_{i}$ é uma matriz de continuidade da partição $\mathcal{X}_{i}$ se

$$
F_{i} x(t)=F_{j} x(t), \quad x(t) \in \mathcal{X}_{i} \cap \mathcal{X}_{j}
$$

Um formato para uma função de Lyapunov contínua quadrática por partes pode ser obtido a seguir.

Lema 4.4 (Johansson, 1999) Seja $\left\{\mathcal{X}_{i}\right\}_{i \in I}$ uma partição poliedral do espaço de estado, e seja $F_{i} \in \mathbb{R}^{p \times n}$ matrizes de continuidade que satisfaçam a Definição 4.3. Então, para cada $T \in \mathbb{R}^{p \times p}$, a função escalar

$$
V(x)=x^{T} F_{i}^{T} T F_{i} x:=x^{T} P_{i} x, \quad x \in \mathcal{X}_{i}
$$


é contínua e quadrática por partes. E ainda, existem $\alpha$ e $\beta$ positivos tais que

$$
\alpha\|x\|_{2}^{2} \leq V(x) \leq \beta\|x\|_{2}^{2}
$$

Note que a escolha das matrizes de continuidade para uma dada partição não é única. Poderia ser utilizado a seguinte matriz de continuidade para todas as partições

$$
F_{i}=\left[I_{p \times n}\right]
$$

porém utilizando a parametrização proposta no Lema 4.4, obtém-se uma única função candidata de Lyapunov globalmente quadrática. Deseja-se, portanto, uma escolha que permita a máxima liberdade na busca da função de Lyapunov. Um procedimento sistemático para encontrar estas matrizes $F_{i}$ pode ser encontrado em Johansson (1999). O procedimento fornecido é diretamente baseado na informação da partição no espaço de estado. Pode-se notar também que as matrizes $F_{i}$ podem sempre ser construídas desde que sejam matrizes de posto coluna completo.

Utilizando (4.5) somente é possível encontrar diferentes $F^{\prime} s$ nas partições que contenham a origem. Para isto não acontecer é necessário o uso de sistemas afins nas demais partições.

Em Johansson (1999) são propostos teoremas de estabilidade utilizando funções de Lyapunov por partes. As informações das partições são integradas nas LMIs através do Procedimento S, porém, não podem ser usados facilmente na síntese de controladores. Para tal, seria necessário o uso de desigualdades matriciais bilineares (BMI, do inglês Bilinear Matrix Inequalities). Para resolver esta dificuldade Feng et al. (2002) introduziu a matriz de Lyapunov $P$ parametrizada como $P_{i}=\left(F_{i}^{T} F_{i}\right)^{-1} F_{i}^{T} T F_{i}\left(F_{i}^{T} F_{i}\right)^{-1}$. A seguir, alguns teoremas principais são apresentados com as respectivas provas para facilitar o entendimento dos resultados apresentados no Capitulo 5.

Teorema 4.5 (Feng et al., 2002) Considere o sistema linear por partes (4.2) com $u(t)=0$. Se existir uma matriz simétrica não singular $T$ tal que

$$
P_{i}=\left(F_{i}^{T} F_{i}\right)^{-1} F_{i}^{T} T F_{i}\left(F_{i}^{T} F_{i}\right)^{-1}, \quad i \in I
$$


e as seguintes LMIs são satisfeitas,

$$
\begin{array}{r}
P_{i}>0, \quad i \in I \\
P_{i} A_{i}^{T}+A_{i} P_{i}<0, \quad i \in I
\end{array}
$$

então o sistema linear por partes é globalmente exponencialmente estável, ou seja, $x(t)$ tende à origem exponencialmente para cada trajetória contínua por partes no espaço de estado.

Prova: Considere a seguinte função candidata de Lyapunov $V(t)$,

$$
V(x)=x^{T} P_{i}^{-1} x, \quad x \in \mathcal{X}_{i}, \quad i \in I .
$$

Segue de (4.7) que

$$
P_{i}^{-1}=F_{i}^{T} T^{-1} F_{i}, \quad i \in I,
$$

então, a função $V(t)$ é contínua através das fronteiras das partições pelo Lema 4.4. Segue de (4.10) e (4.8) que existem constantes $\alpha>0$ e $\beta>0$ tais que

$$
\alpha\|x\|^{2} \leq V(t) \leq \beta\|x\|^{2}
$$

Em adição, (4.9) implica que existe uma constante $\rho>0$ tal que

$$
A_{i}^{T} P_{i}^{-1}+P_{i}^{-1} A_{i}+\rho I<0 .
$$

Então, ao longo das trajetórias do sistema, tem-se

$$
\begin{aligned}
\dot{V}(t) & =x^{T}\left[A_{i}^{T} P_{i}^{-1}+P_{i}^{-1} A_{i}\right] x \\
& \leq x^{T}(-\rho I) x \\
& =-\rho\|x\|^{2} .
\end{aligned}
$$

O resultado desejado segue diretamente de (4.11) e (4.12) baseado no Lema(4.1). 


\section{Exemplo 4.3 (Análise de estabilidade usando funções de Lyapunov} por partes) Considere o sistema do Exemplo 4.2, uma alternativa para resolver o problema de análise de estabilidade é o uso de funções de Lyapunov por partes. Utilizando o Teorema 4.5 obteve-se a factibilidade das LMIs (4.8) e (4.9) encontrando

$$
\begin{aligned}
T & =\left[\begin{array}{rrr}
355.09 & -328.80 & -80.68 \\
-328.80 & 354.60 & 72.69 \\
-80.68 & 72.69 & 63.73
\end{array}\right], \\
P_{1}= & {\left[\begin{array}{cc}
341.82 & 76.69 \\
76.69 & 63.73
\end{array}\right] } \\
P_{2}= & {\left[\begin{array}{rr}
13.02 & -3.99 \\
-3.99 & 63.73
\end{array}\right] . }
\end{aligned}
$$

Foram utilizados as seguintes matrizes de continuidade

$$
\begin{aligned}
& F_{1}=\left[\begin{array}{rr}
-1 & 0 \\
1 & 0 \\
0 & 1
\end{array}\right], \\
& F_{2}=\left[\begin{array}{ll}
1 & 0 \\
1 & 0 \\
0 & 1
\end{array}\right] .
\end{aligned}
$$

\subsection{Projeto de Controladores}

Até o presente momento estudou-se a representação de um sistema não linear num sistema linear por partes e na análise deste sistema. O objetivo agora é encontrar uma lei de controle para tornar o sistema em malha fechada estável. A primeira tentativa é encontrar um controlador via lei de controle de realimentação de estado, $u=K x$, e obter desigualdades que possam ser resolvidas via LMIs.

A partir do Teorema 4.5 é possível obter um controlador, via realimentação de estado, que estabilize o sistema linear por partes (4.2). O controlador proposto é baseado no modelo linear local definido em cada partição. 
Considera-se o seguinte controlador contínuo por partes

$$
u(t)=K_{i} x(t), \quad x(t) \in \mathcal{X}_{i}, \quad i \in I
$$

Através da lei de controle acima, o sistema em malha fechada é obtido combinando o sistema linear por partes

$$
\dot{x}(t)=A_{i} x(t)+B_{i} u(t), \quad x(t) \in \mathcal{X}_{i}, \quad i \in I
$$

e o controlador (4.13), podendo ser descrito pela seguinte equação em cada partição como,

$$
\dot{x}=A_{c i} x(t), \quad x(t) \in \mathcal{X}_{i}, \quad i \in I
$$

onde

$$
A_{c i}=A_{i}+B_{i} K_{i}
$$

Para obter o controlador segue o seguinte teorema.

Teorema 4.6 O sistema linear por partes (4.14) com $x(t) \in \cup_{i \in I} \mathcal{X}_{i}, i \in I$, é globalmente exponencialmente estável se existir uma matriz simétrica não singular $T$, e matrizes $Q_{i}$, tais que,

$$
P_{i}=\left(F_{i}^{T} F_{i}\right)^{-1} F_{i}^{T} T F_{i}\left(F_{i}^{T} F_{i}\right)^{-1}, \quad i \in I
$$

satisfazem as seguintes LMIs

$$
\begin{aligned}
P_{i} & >0 \\
P_{i} A_{i}^{T}+A_{i} P_{i}+Q_{i}^{T} B_{i}^{T}+B_{i} Q_{i} & <0, \quad i \in I .
\end{aligned}
$$

O controlador de cada subsistema local é dado por

$$
K_{i}=Q_{i} P_{i}^{-1}, \quad i \in I
$$

Prova: Segue da aplicação da lei de controle (4.13) no Teorema 4.5. 


\subsection{Sistemas Fuzzy com Funções de Lyapunov por Partes}

A lógica fuzzy para controle tem provado ser uma ferramenta satisfatória na solução de problemas não-lineares complexos. Como foi visto em em capítulo anterior a modelagem fuzzy decompõe o sistema não-linear em diversos subsistemas lineares com as respectivas funções de pertinência de acordo com o conhecimento heurístico do problema e utiliza uma lei de controle simples para emular a estratégia de controle humano em cada região de operação. O controle global é construído combinando os controles locais através das funções de pertinência fuzzy.

Foi visto também que a técnica de modelagem fuzzy Takagi-Sugeno possibilita a modelagem matemática e a síntese em termos de LMIs dos controladores locais. É necessário que uma matriz comum positiva definida $P$ fosse encontrada satisfazendo as LMIs dos modelos locais. Contudo, este pode ser um problema difícil de resolver uma vez que tal matriz pode não existir, especialmente para sistemas não-lineares altamente complexos.

A idéia de se utilizar funções de Lyapunov por partes em sistemas fuzzy tem sido recentemente abordada por alguns autores como forma de tornar o problema de busca de uma função de Lyapunov menos conservadora. O objetivo é a síntese de controladores locais para cada partição do espaço de estado utilizando funções de Lyapunov por partes para alcançar a estabilidade global do sistema resultante fuzzy em malha fechada. Para tal é necessário garantir a continuidade da função na fronteira das partições como desenvolvido nos sistemas lineares por partes. As funções de Lyapunov por partes constituem uma classe de funções candidatas muito mais rica que funções de Lyapunov comuns.

\subsubsection{Estabilidade e Síntese de Controladores}

O uso de regras em sistemas fuzzy leva a uma divisão do espaço de estado análogo ao que se obtém em sistemas lineares por partes. Cada região é descrita por um 
sistema linear e de modo que a influência das dinâmicas das partições adjacentes, definida pelas funções de pertinência, é sempre menor do que a pertinência da dinâmica local. Cada partição, portanto, tem sua fronteira no ponto de cruzamento das funções de pertinência, onde elas se igualam e somadas resultam em 1.

Como no caso de sistemas lineares por partes, é proposto por Johansson et al. (1999) um teorema de análise de estabilidade menos conservador, utilizando funções de Lyapunov por partes em sistemas fuzzy e considerando as informações das partições nas LMIs, porém, é complexo resolver o problema de síntese do controlador sem recorrer a BMIs. Mais uma vez é necessária uma outra abordagem para a estabilidade.

A fim de introduzir o novo resultado definem-se as $r$ partições do espaço de estado como segue. A Figura 4.3 ilustra como são definidas as partições.

As partições fuzzy são descritas pelos conjuntos

$$
\overline{\mathcal{S}}_{i}=\mathcal{S}_{i} \cup \partial \mathcal{S}_{i}, \quad i=1,2, \ldots, r
$$

onde

$$
\mathcal{S}_{i}=\left\{x \mid \mu_{i}(x)>\mu_{j}(x), \quad i=1,2, \ldots, r, j \neq i\right\}
$$

e sua fronteira

$$
\partial \mathcal{S}_{i}=\left\{x \mid \mu_{i}(x)=\mu_{j}(x), \quad i=1,2, \ldots, r, j \neq i\right\}
$$

O modelo fuzzy pode ser expresso pelo seguinte modelo global

$$
\dot{x}(t)=A(\mu(x)) x(t)+B(\mu(x)) u(t),
$$

no qual

$$
\begin{aligned}
& A(\mu(x))=\sum_{i=1}^{r} \mu_{i}(x) A_{i} \\
& B(\mu(x))=\sum_{i=1}^{r} \mu_{i}(x) B_{i} .
\end{aligned}
$$

Para o uso de funções de Lyapunov por partes, cada partição $\overline{\mathcal{S}}_{i}$ do espaço de estado terá sua própria função de Lyapunov. O sistema global fuzzy será 


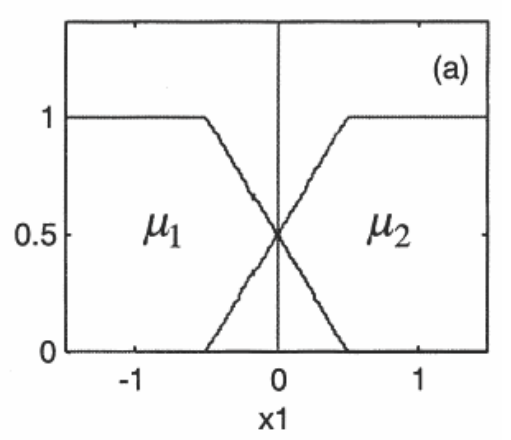

(a)

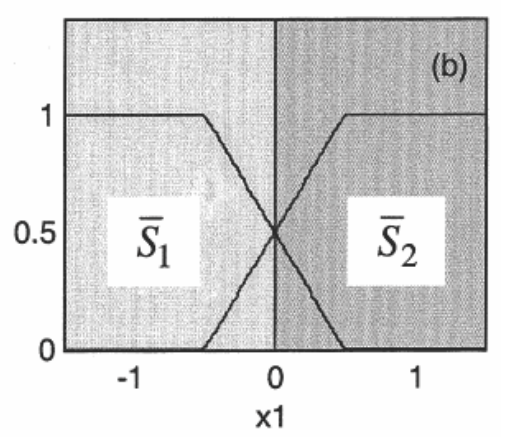

(b)

Figura 4.3: (a) Funções de pertinência. (b) Divisão no espaço de estado em partições. reescrito para cada partição e as influências das dinâmicas de partições adjacentes do espaço de estado serão vistas como incertezas, com um limitante superior conhecido. Pode-se descrever o modelo fuzzy (4.16), em cada partição, pelo $i$ ésimo subsistema

$$
\begin{array}{r}
\dot{x}(t)=\left(A_{i}+\Delta A_{i}(\mu)\right) x(t)+\left(B_{i}+\Delta B_{i}(\mu)\right) u(t), \quad x(t) \in \overline{\mathcal{S}}_{i}, \\
i=1, \ldots, r,
\end{array}
$$

onde

$$
\begin{aligned}
\Delta A_{i}(\mu) & =\sum_{j \in \mathcal{H}_{i}} \mu_{j} \Delta A_{i j} \\
\Delta B_{i}(\mu) & =\sum_{j \in \mathcal{H}_{i}} \mu_{j} \Delta B_{i j}
\end{aligned}
$$


$\mathrm{e}$

$$
\begin{aligned}
\Delta A_{i j} & =A_{j}-A_{i}, \\
\Delta B_{i j} & =B_{j}-B_{i}, \\
\mathcal{H}_{i}: & =\left\{j \mid \mu_{j} \neq 0, \mu_{j} \geq \mu_{i}\right\},
\end{aligned}
$$

ou seja, $\mathcal{H}_{i}$ é o conjunto contendo os índices das funções de pertinência que não são iguais a zero quando o $i$-ésimo subsistema tem papel dominante. Note que $\Delta A_{i}$ e $\Delta B_{i}$ representam os termos de incerteza do subsistema $i$ devido as interações de outros subsistemas quando o modelo global é representado.

Como mostrado acima, é possível descrever a influência de uma dinâmica predominante numa região em outra como incerteza. A fim de eliminar esta incerteza em (4.17) é possível definir os seguintes limitantes superiores:

$$
\begin{aligned}
{\left[\Delta A_{i}(\mu)\right]\left[\Delta A_{i}(\mu)\right]^{T} } & \leq E_{i A} E_{i A}^{T} \\
{\left[\Delta B_{i}(\mu)\right]\left[\Delta B_{i}(\mu)\right]^{T} } & \leq E_{i B} E_{i B}^{T} .
\end{aligned}
$$

Pode-se determinar estes limitantes superiores de várias formas visto que estas incertezas são conhecidas a priori. Uma maneira simples segue abaixo:

$$
E_{i A} E_{i A}^{T}=\left(\sum_{j \in \mathcal{H}_{i}} \mu_{j}(\bar{x}) \Delta A_{i j}\right)\left(\sum_{j \in \mathcal{H}_{i}} \mu_{j}(\bar{x}) \Delta A_{i j}\right)^{T}
$$

onde $\bar{x}$ é o estado tal que $\mu_{i}(\bar{x})=0,5$.

Teorema 4.7 (Feng, 2003) Considere o sistema fuzzy (4.17) com $u=0$. Se existir um conjunto de constantes positivas $\epsilon_{i}, i=1,2, \ldots, r$, e uma matriz simétrica T tal que com

$$
P_{i}=\left(F_{i}^{T} F_{i}\right)^{-1} F_{i}^{T} T F_{i}\left(F_{i}^{T} F_{i}\right)^{-1}, \quad i=1,2, \ldots, r
$$

as seguintes LMIs são satisfeitas

$$
\begin{aligned}
& P_{i}>0 \\
& {\left[\begin{array}{cc}
P_{i} A_{i}^{T}+A_{i} P_{i}+\epsilon E_{i A} E_{i A}^{T} & P_{i} \\
P_{i} & -\epsilon I
\end{array}\right]<0, \quad i=1,2, \ldots, r}
\end{aligned}
$$


então o sistema fuzzy é globalmente exponencialmente estável, isto é, $x(t)$ tende a origem exponencialmente para toda trajetória contínua por partes no espaço de estado.

Prova: Considere a função candidata de Lyapunov $V(t)$ :

$$
V(t)=x^{T} P_{i}^{-1} x, \quad x \in \overline{\mathcal{S}}_{i}, \quad i=1,2, \ldots, r .
$$

Segue de (4.20) que

$$
P_{i}^{-1}=F_{i}^{T} T^{-1} F_{i}, \quad i=1,2, \ldots, r
$$

então a função $V(t)$ é contínua através das fronteiras das partições. De (4.21) e (4.23) segue que existe constantes $\alpha>0$ e $\beta>0$ tais que

$$
\alpha\|x\|^{2} \leq V(t) \leq \beta\|x\|^{2} .
$$

Para alguma constante $\epsilon>0$ e matrizes $X$ e $Y$ de dimensões apropriadas, tem-se (Petersen, 1987)

$$
X^{T} Y+Y^{T} X \leq \frac{1}{\epsilon} X^{T} X+\epsilon Y^{T} Y
$$

então, pode-se mostrar facilmente que

$$
P_{i}\left(A_{i}+\Delta A_{i}(\mu)\right)^{T}+\left(A_{i}+\Delta A_{i}(\mu)\right) P_{i} \leq P_{i} A_{i}^{T}+A_{i} P_{i}+\frac{1}{\epsilon_{i}} P_{i} P_{i}+\epsilon_{i} E_{i A} E_{i A}^{T},
$$

para $E_{i A} E_{i A}^{T}$ um limitante superior para $\Delta A_{i}(\mu) \Delta A_{i}^{T}(\mu)$.

Em adição, via complemento de Schur, segue de (4.22) que

$$
P_{i} A_{i}^{T}+A_{i} P_{i}+\frac{1}{\epsilon_{i}} P_{i} P_{i}+\epsilon_{i} E_{i A} E_{i A}^{T}<0 .
$$

Então, segue de (4.26) e (4.27) que

$$
P_{i}\left(A_{i}+\Delta A_{i}(\mu)\right)^{T}+\left(A_{i}+\Delta A_{i}(\mu)\right) P_{i}<0 .
$$

Isto implica que

$$
\left(A_{i}+\Delta A_{i}(\mu)\right)^{T} P_{i}^{-1}+P_{i}^{-1}\left(A_{i}+\Delta A_{i}(\mu)\right)<0
$$


que garante a existência de uma constante $\rho>0$ tal que

$$
\left(A_{i}+\Delta A_{i}(\mu)\right)^{T} P_{i}^{-1}+P_{i}^{-1}\left(A_{i}+\Delta A_{i}(\mu)\right)+\rho I<0 .
$$

Então, ao longo das trajetórias do sistema, tem-se

$$
\begin{aligned}
\dot{V}(t) & =x^{T}\left[\left(A_{i}+\Delta A_{i}(\mu)\right)^{T} P_{i}^{-1}+P_{i}^{-1}\left(A_{i}+\Delta A_{i}(\mu)\right)\right] x \\
& \leq x^{T}(-\rho I) x \\
& =-\rho\|x\|^{2} .
\end{aligned}
$$

Portanto, o resultado desejado segue diretamente de (4.24) e (4.28) pelo Lema 4.1.

Considere o controlador por partes:

$$
u(t)=K(x) x(t):=K_{i} x(t), \quad x(t) \in \overline{\mathcal{S}_{i}}, \quad i=1,2, \ldots, r .
$$

O sistema global fuzzy em malha fechada pode ser descrito, em cada partição, pelo $i$-ésimo subsistema:

$$
\dot{x}(t)=A_{c i}(\mu) x(t), \quad x(t) \in \overline{\mathcal{S}_{i}}, \quad i=1,2, \ldots, r,
$$

no qual

$$
A_{c i}(\mu)=A_{i}+\Delta A_{i}(\mu)+\left(B_{i}+\Delta B_{i}(\mu)\right) K_{i}
$$

O controlador proposto é chaveado no espaço de estado e, portanto, se mantém constante em cada partição. Deve satisfazer as condições de estabilidade e desempenho do sistema fuzzy e para tal, é necessário considerar as influências dos outros subsistemas. Tem-se o resultado.

Teorema 4.8 (Feng, 2003) O sistema (4.30) é globalmente estável se existirem constantes $\epsilon_{i}>0, i=1,2, \ldots, r$, matrizes de continuidade $F_{i}, i=1,2, \ldots, r$, uma matriz simétrica $T$, e um conjunto de matrizes $Q_{i}, i=1,2, \ldots, r$ tais que com

$$
P_{i}=\left(F_{i}^{T} F_{i}\right)^{-1} F_{i}^{T} T F_{i}\left(F_{i}^{T} F_{i}\right)^{-1}, \quad i=1,2, \ldots, r
$$


as seguintes LMIs são satisfeitas

$$
\begin{aligned}
& P_{i}>0 \\
& {\left[\begin{array}{ccc}
\Omega_{i} & P_{i} & Q_{i}^{T} \\
P_{i} & -\epsilon_{i} I & 0 \\
Q_{i} & 0 & -\epsilon_{i} I
\end{array}\right]<0, \quad i=1,2, \ldots, r}
\end{aligned}
$$

onde

$$
\Omega_{i}=P_{i} A_{i}^{T}+A_{i} P_{i}+Q_{i}^{T} B_{i}^{T}+B_{i} Q_{i}+\epsilon_{i}\left(E_{i A} E_{i A}^{T}+E_{i B} E_{i B}^{T}\right) .
$$

E o ganho do controlador por partes (4.29) em cada subsistema local é dado por

$$
K_{i}=Q_{i} P_{i}^{-1}, \quad i=1,2, \ldots, r
$$

Prova: O sistema fuzzy em malha fechada (4.30) é globalmente estável se, pelo Teorema 4.7,

$$
\begin{gathered}
\left(A_{i}+\Delta A_{i}(\mu)+\left(B_{i}+\Delta B_{i}(\mu)\right) K_{i}\right) P_{i}+P_{i}\left(A_{i}+\Delta A_{i}(\mu)+\left(B_{i}+\Delta B_{i}(\mu)\right) K_{i}\right)^{T}<0 \\
i=1,2, \ldots, r .
\end{gathered}
$$

Manipulando a desigualdade acima, fazendo $Q_{i}=K_{i} P_{i}$ e utilizando (4.25) e os limitantes (4.18) tem-se

$$
\begin{aligned}
& A_{i} P_{i}+P_{i} A_{i}^{T}+B_{i} Q_{i}+Q_{i}^{T} B_{i}^{T}+\Delta A_{i}(\mu) P_{i}+P_{i} \Delta A_{i}(\mu)^{T} \\
& +\Delta B_{i}(\mu) Q_{i}+Q_{i}^{T} \Delta B_{i}(\mu)^{T} \\
\leq & A_{i} P_{i}+P_{i} A_{i}^{T}+B_{i} Q_{i}+Q_{i}^{T} B_{i}^{T}+\frac{1}{\epsilon_{i}} P_{i} P_{i}+\epsilon_{i} \Delta A_{i}(\mu) \Delta A_{i}(\mu)^{T} \\
& +\frac{1}{\epsilon_{i}} Q_{i}^{T} Q_{i}+\epsilon_{i} \Delta B_{i}(\mu) \Delta B_{i}(\mu)^{T} \\
\leq & A_{i} P_{i}+P_{i} A_{i}^{T}+B_{i} Q_{i}+Q_{i}^{T} B_{i}^{T}+\epsilon_{i}\left(E_{A i} E_{A i}^{T}+E_{B i} E_{B i}^{T}\right) \\
& +\frac{1}{\epsilon_{i}} P_{i} P_{i}+\frac{1}{\epsilon_{i}} Q_{i}^{T} Q_{i}<0 \\
& i=1,2, \ldots, r .
\end{aligned}
$$

Aplicando por duas vezes o complemento de Schur em (4.33) obtém-se (4.32).

Exemplo 4.4 (Projeto de um controle fuzzy) Considere o sistema fuzzy T-S abaixo com 4 regras e as funções de pertinência mostradas na Figura 4.4. 


\section{Regra do Modelo 1:}

$S E$

$x_{1}(t)$ é "Muito Negativo",

ENT $\tilde{A} O \quad \dot{x}(t)=A_{1} x(t)+B(t) u(t)$

Regra do Modelo 2:

$S E$

$x_{1}(t)$ é "Negativo",

ENT $\tilde{A} O \quad \dot{x}(t)=A_{2} x(t)+B(t) u(t)$

\section{Regra do Modelo 3:}

$S E$

$$
x_{1}(t) \text { é "Positivo", }
$$

ENT $\tilde{A} O \quad \dot{x}(t)=A_{3} x(t)+B(t) u(t)$

Regra do Modelo 4:

$S E$

$x_{1}(t)$ é "Muito Positivo",

ENT $\tilde{A} O \quad \dot{x}(t)=A_{4} x(t)+B(t) u(t)$

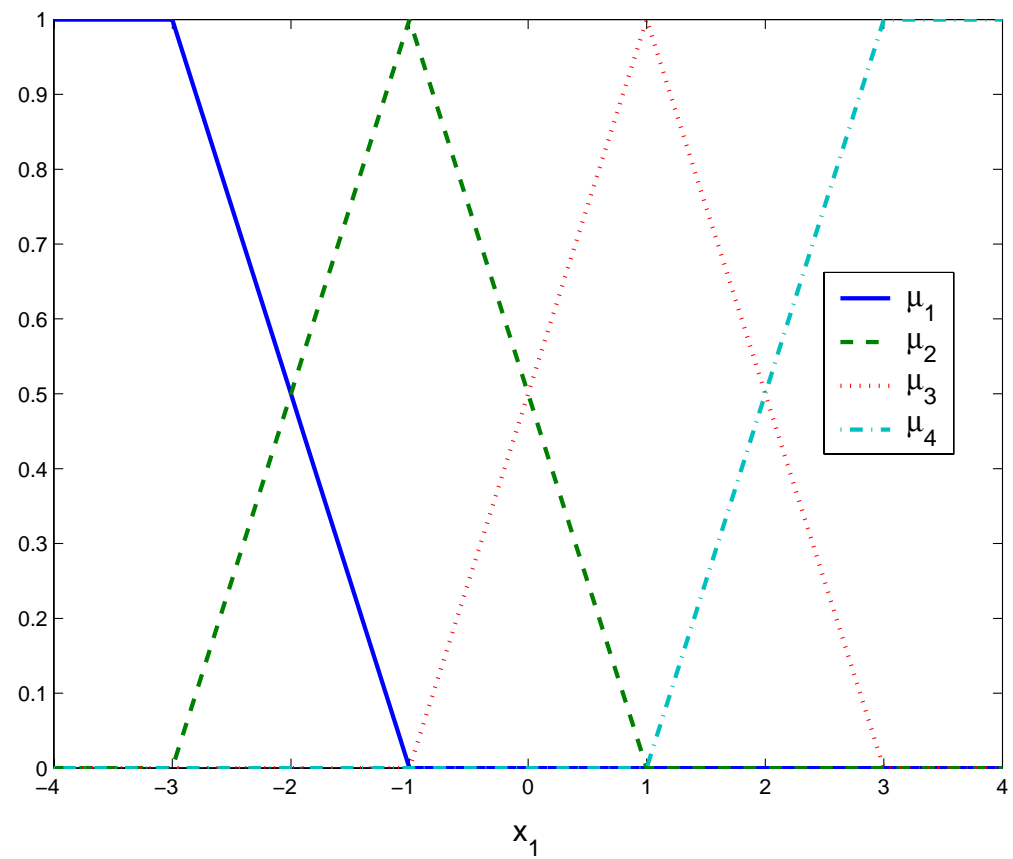

Figura 4.4: Funções de pertinência fuzzy das variáveis linguísticas "Muito Negativo", "Negativo", "Positivo"e "Muito Positivo"representadas pelas variáveis $\mu_{1}, \mu_{2}, \mu_{3}$ e $\mu_{4}$ respectivamente. 
As matrizes de estado são

$$
\begin{array}{ll}
A_{1}= & {\left[\begin{array}{cccc}
0 & 1 & 0 & 0 \\
0 & 0 & -7.129 & -1.429 \\
0 & 0 & 0 & 1 \\
0 & 0 & 0 & 0
\end{array}\right], A_{2}=\left[\begin{array}{cccc}
0 & 1 & 0 & 0 \\
0 & 0 & -7.129 & -0.1428 \\
0 & 0 & 0 & 1 \\
0 & 0 & 0 & 0
\end{array}\right],} \\
A_{3}= & {\left[\begin{array}{cccc}
0 & 1 & 0 & 0 \\
0 & 0 & -7.129 & 0.1428 \\
0 & 0 & 0 & 1 \\
0 & 0 & 0 & 0
\end{array}\right], A_{4}=\left[\begin{array}{cccc}
0 & 1 & 0 & 0 \\
0 & 0 & -7.129 & 1.429 \\
0 & 0 & 0 & 1 \\
0 & 0 & 0 & 0
\end{array}\right],}
\end{array}
$$

$e$

$$
B=\left[\begin{array}{l}
0 \\
0 \\
0 \\
1
\end{array}\right] .
$$

A fim de obter funções de Lyapunov quadráticas por partes dividiu-se o espaço de estado nas partições $\overline{\mathcal{S}}_{1}=\left\{x: x_{1} \in\left[\begin{array}{ll}-4 & -2\end{array}\right]\right\}, \overline{\mathcal{S}}_{2}=\left\{x: x_{1} \in\left[\begin{array}{ll}-2 & 0\end{array}\right]\right\}$, $\overline{\mathcal{S}}_{3}=\left\{x: x_{1} \in\left[\begin{array}{ll}0 & 2\end{array}\right]\right\}$ e $\overline{\mathcal{S}}_{4}=\left\{x: x_{1} \in\left[\begin{array}{ll}2 & 4\end{array}\right]\right\}$ de acordo com as funções de pertinência do sistema fuzzy, conforme ilustra a Figura 4.5.

Para garantir a continuidade da função de Lyapunov segundo o Lema 4.4 escolheu-se as funções de continuidade $F_{1}, F_{2}, F_{3}$ e $F_{4}$ para as partições. A metodologia para encontrar tais funções está descrito em Johansson et al. (1999).

$$
F_{1}=F_{2}=\left[\begin{array}{cccc}
-1 & 0 & 0 & 0 \\
1 & 0 & 0 & 0 \\
0 & 1 & 0 & 0 \\
0 & 0 & 1 & 0 \\
0 & 0 & 0 & 1
\end{array}\right], \quad F_{3}=F_{4}=\left[\begin{array}{cccc}
1 & 0 & 0 & 0 \\
1 & 0 & 0 & 0 \\
0 & 1 & 0 & 0 \\
0 & 0 & 1 & 0 \\
0 & 0 & 0 & 1
\end{array}\right] .
$$

As LMIs (4.32) foram factiveis e forneceram os seguintes controladores por 


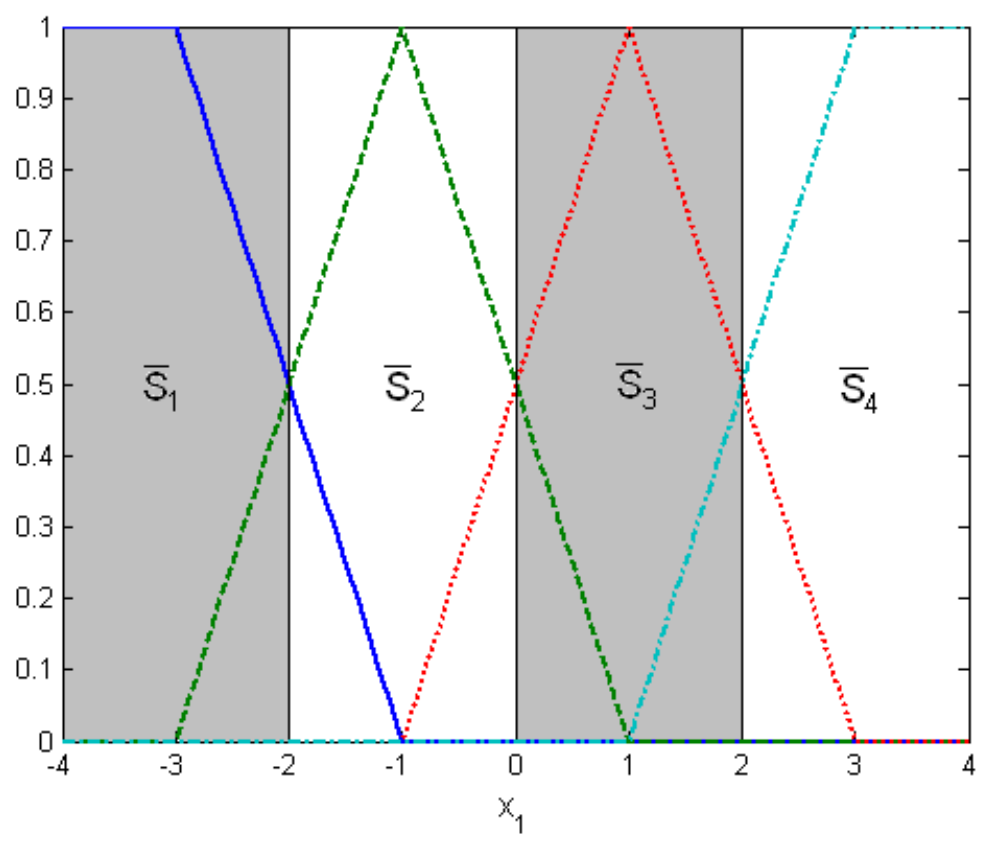

Figura 4.5: Partições no espaço de estados.

partes

$$
\begin{aligned}
& K_{1}=\left[\begin{array}{llll}
9.987 & 19.78 & -86.70 & -22.00
\end{array}\right] \\
& K_{2}=\left[\begin{array}{llll}
9.982 & 19.77 & -86.66 & -21.99
\end{array}\right] \\
& K_{3}=\left[\begin{array}{llll}
14.56 & 27.76 & -132.80 & -28.35
\end{array}\right] \\
& K_{4}=\left[\begin{array}{llll}
14.55 & 27.73 & -132.67 & -28.33
\end{array}\right]
\end{aligned}
$$


e matrizes

$$
\begin{aligned}
& T=\left[\begin{array}{ccccc}
420.68 & -0.0856 & 0.2561 & 0.0761 & -0.0967 \\
-0.0856 & -417.51 & -1.2665 & -0.0964 & -0.0283 \\
0.2561 & -1.2665 & 1.4504 & 0.2719 & -0.1128 \\
0.0761 & -0.0964 & 0.2719 & 0.0749 & -0.0900 \\
-0.0967 & -0.0283 & -0.1128 & -0.0900 & 0.3140
\end{array}\right], \\
& P_{1}=P_{2}=\left[\begin{array}{cccc}
0.8344 & -0.7613 & -0.0863 & 0.0342 \\
-0.7613 & 1.4504 & 0.2719 & -0.1128 \\
-0.0863 & 0.2719 & 0.0749 & -0.0900 \\
0.0342 & -0.1128 & -0.0900 & 0.3140
\end{array}\right] \text {, } \\
& P_{3}=P_{4}=\left[\begin{array}{cccc}
0.7488 & -0.5052 & -0.0102 & -0.0625 \\
-0.5052 & 1.4504 & 0.2719 & -0.1128 \\
-0.0102 & 0.2719 & 0.0749 & -0.0900 \\
-0.0625 & -0.1128 & -0.0900 & 0.3140
\end{array}\right] \text {, } \\
& Q_{1}=\left[\begin{array}{llll}
0.0000 & 0.0002 & -0.0001 & -0.9986
\end{array}\right], \\
& Q_{2}=\left[\begin{array}{llll}
0.0001 & -0.0001 & -0.0001 & -0.9986
\end{array}\right] \text {, } \\
& Q_{3}=\left[\begin{array}{llll}
0.0003 & -0.0003 & -0.0001 & -0.9986
\end{array}\right] \text {, } \\
& Q_{4}=\left[\begin{array}{llll}
0.0002 & -0.0008 & -0.0002 & -0.9980
\end{array}\right] \text {. }
\end{aligned}
$$

Observe que devido ao fato de $F_{1}=F_{2}$ e $F_{3}=F_{4}$ a função de Lyapunov só se modifica na mudança das partições $\overline{\mathcal{S}}_{2}$ e $\overline{\mathcal{S}}_{3}$, mas mantendo a continuidade na fronteira.

A fim de simular o sistema em malha fechada constrói-se os diagramas Simulink representados nas Figuras 4.6-4.9. A resposta temporal mostrou-se satisfatória, conforme pode ser visto na Figura 4.10. 


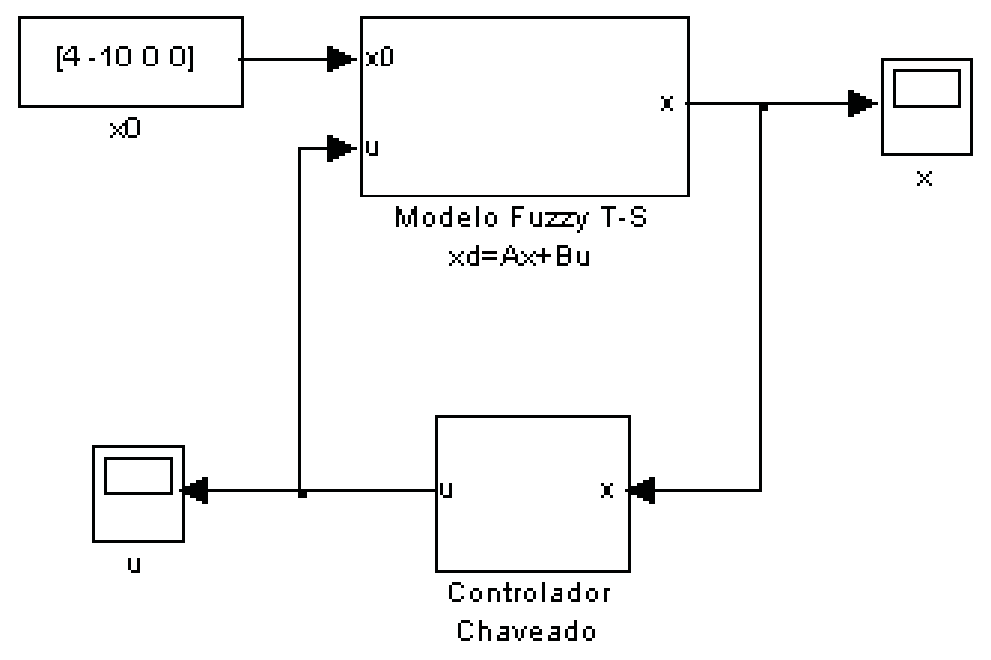

Figura 4.6: Sistema em malha fechada.

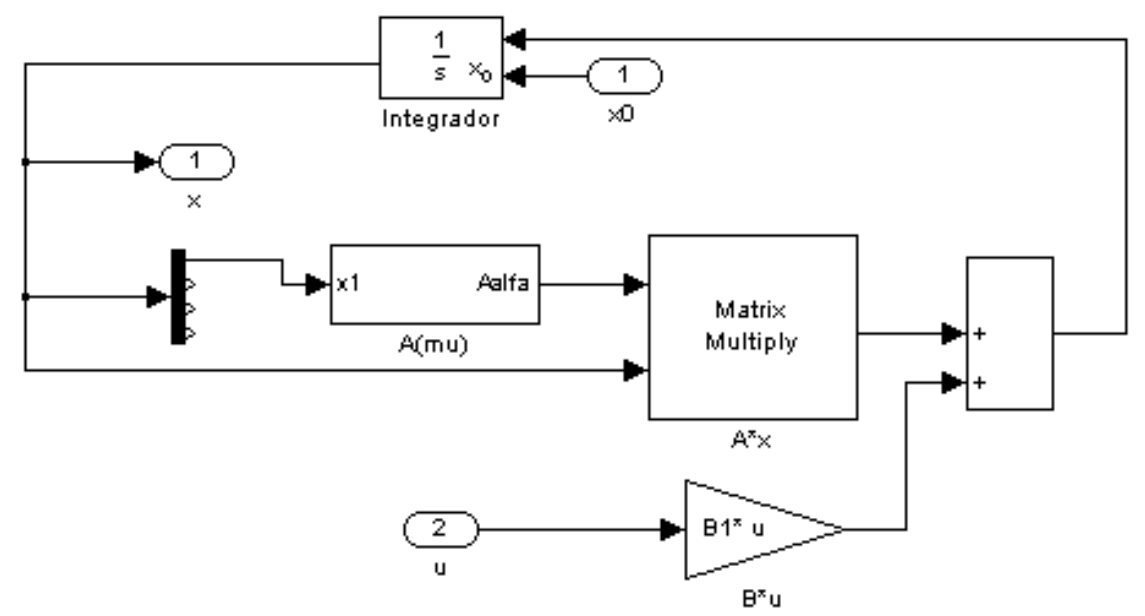

Figura 4.7: Modelo fuzzy T-S. 


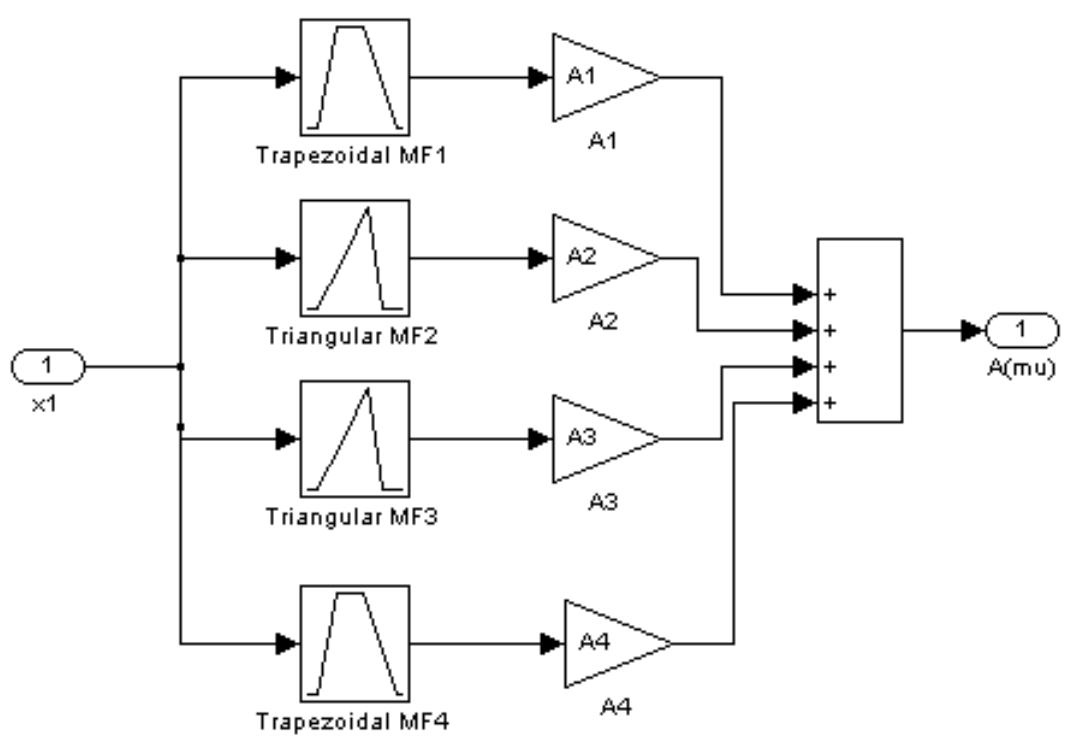

Figura 4.8: Matriz de estado $A(\mu)$ ponderada.

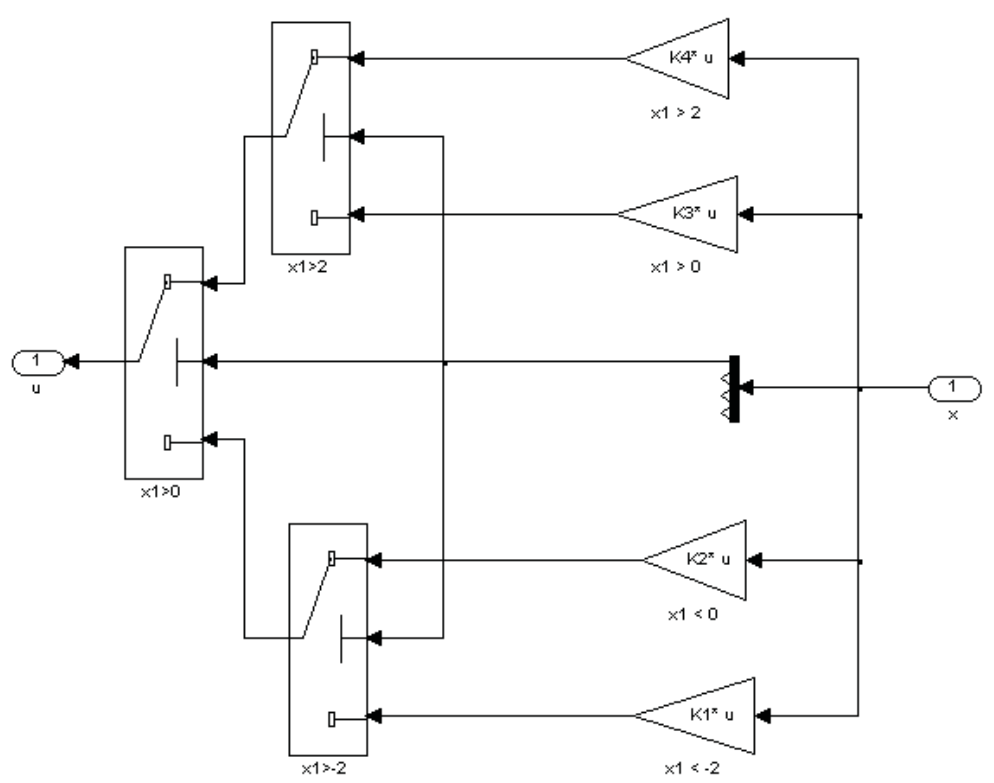

Figura 4.9: Controlador chaveado. 


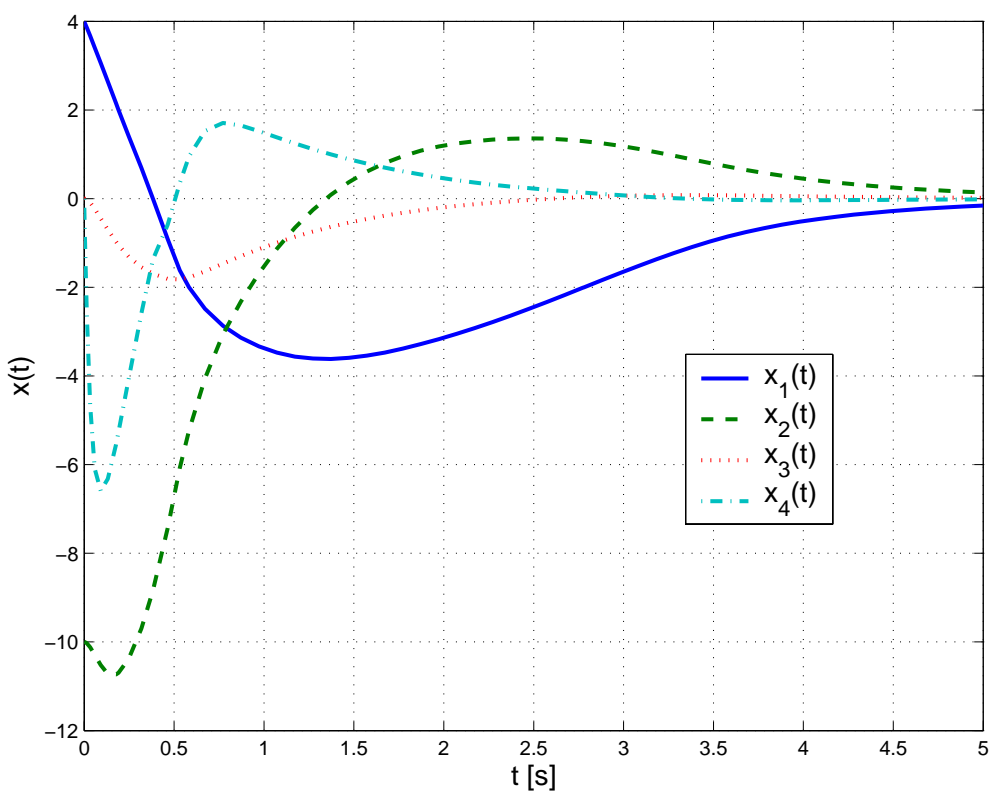

Figura 4.10: Resposta do estado $\left[\begin{array}{llll}x_{1} & x_{2} & x_{3} & x_{4}\end{array}\right]^{T}$ à condição inicial $x_{0}=\left[\begin{array}{llll}4 & -10 & 0 & 0\end{array}\right]$. 


\section{Capítulo 5}

\section{Sistemas Fuzzy com Restrição de}

\section{Alocação de Pólos}

Foram mostrados em capítulos anteriores as vantagens da utilização da modelagem fuzzy para sistemas não-lineares. Com esta modelagem é possível dividir a dinâmica no espaço de estado em modelos lineares locais e através de funções de pertinência usar o conhecimento humano para melhor descrever o sistema. Esta importância atribuída às técnicas de modelagem justifica-se, pois é através destas que será possível prever o comportamento do sistema e controlá-lo de forma satisfatória. Porém, apenas estabilizar o sistema não é suficiente, é necessário que este tenha um comportamento adequado no que diz respeito a insensibilidade a ruídos e incertezas paramétricas, resposta transitória rápida ou com pouco sobre-sinal e outros aspectos de importância. Há várias técnicas de controle que possibilitam alcançar um desempenho satisfatório como as técnicas $H_{2}, H_{\infty}$, síntese $\mu$ entre outras. Dá-se destaque, no presente capítulo, ao uso da alocação de pólos em sistemas fuzzy. A estabilidade é alcançada com os pólos no semi-plano esquerdo do plano-s, porém, sabe-se que o posicionamento dos pólos está relacionado diretamente com a resposta transitória do sistema, conforme discutido na Seção 2.3.4.

Os resultados existentes em alocação de pólos em sistemas fuzzy contribuiram para o desenvolvimento nas técnicas apresentadas neste capítulo. Os métodos apresentados em Joh et al. (1997) e em Hong e Nam (2003) foram modificados, 
de forma a remover redundâncias nas LMIs, resultando no Teorema 5.3.

A abordagem proposta por Johansson et al. (1999) inspirou as técnicas de uso de funções de Lyapunov por partes em sistemas fuzzy apresentadas em Feng e Sun (2002), Feng (2003), Feng (2004) e Feng et al. (2005). Nesses trabalhos os modelos fuzzy são reescritos como em (4.17) para a síntese do controlador. Entretanto, não há nenhum trabalho envolvendo funções de Lyapunov por partes e contínuas no espaço de estado no problema de alocação de pólos utilizando a modelagem fuzzy.

Para modelos lineares locais que contenham pontos de equilíbrio do sistema não-linear a $\mathcal{D}$-estabilidade garante a estabilidade do ponto de equilíbrio e uma convergência exponencial adequada. Para modelos locais de regiões de nãoequilíbrio não é possível falar na estabilidade do ponto de equilíbrio deste sistema local uma vez que ele não é coincidente com os do sistema original, conforme visto na Seção 3.1.1. Então, a $\mathcal{D}$-estabilidade deste sistema está relacionada com a convergência das trajetórias de (3.11) para a origem, ou seja, as órbitas tendem a se aproximar com as restrições dinâmicas (sobre-sinal, tempo de acomodação, freqüência de oscilação) impostas pela localização dos pólos.

Para o caso de chaveamento do controlador na mudança de partição $\mathcal{S}_{i}, i=$ $1,2, \ldots, r$, a restrição dinâmica da resposta temporal ocorre sempre que o tempo de permanência (em inglês, dwell time) for longo o suficiente para permitir a acomodação de cada modo (Liberzon, 2003).

Para um modelo linear a $\mathcal{D}$-estabilidade implica a alocação apropriada dos pólos do sistema e é um conceito bem conhecido. O modelo fuzzy Takagi-Sugeno é um modelo não-linear e portanto faz-se necessário uma definição adequada de $\mathcal{D}$-estabilidade.

Definição 5.1 (D-Estabilidade de Modelos Fuzzy Takagi-Sugeno) O modelo global fuzzy Takagi-Sugeno (4.16) é dito ser $\mathcal{D}$-estável se os autovalores das combinações fuzzy das matrizes de estado dos modelos locais, $\sum_{i=1}^{r} \mu_{i}(x) A_{i}$, para todo $x$ pertencente ao universo de discurso ${ }^{1}$, pertencer a região $L M I \mathcal{D} \subset \mathbb{C}$.

\footnotetext{
${ }^{1}$ Será chamado de universo de discurso o conjunto de todos os possíveis valores que as variáveis premissas podem assumir.
} 
A seguir propõe-se o lema abaixo que garante a $\mathcal{D}$-estabilidade do sistema fuzzy.

Lema 5.2 O sistema fuzzy Takagi-Sugeno (4.16) é D-estável se existir uma matriz simétrica $P>0$ satisfazendo

$$
L \otimes P+M \otimes\left(P A_{i}\right)+M^{T} \otimes\left(A_{i}^{T} P\right)<0, \quad i=1,2, \ldots, r
$$

onde as matrizes $L$ e $M$ são escolhidas convenientemente de forma a definir a região do plano-s no qual os pólos devem pertencer.

Prova: Multiplicando (5.1) pela função de pertinência $\mu_{i}(x)$ tem-se

$$
\mu_{i} L \otimes P+\mu_{i} M \otimes\left(P A_{i}\right)+\mu_{i} M^{T} \otimes\left(A_{i}^{T} P\right)<0, \quad i=1,2, \ldots, r .
$$

Usando a seguinte propriedade $\alpha A \otimes B=A \otimes(\alpha B)$, tem-se

$$
\mu_{i}(L \otimes P)+M \otimes\left(P \mu_{i} A_{i}\right)+M^{T} \otimes\left(\mu_{i} A_{i}^{T} P\right)<0
$$

que pode ser escrita como

$$
\sum_{i=1}^{r} \mu_{i}(L \otimes P)+M \otimes\left(P \sum_{i=1}^{r} \mu_{i} A_{i}\right)+M^{T} \otimes\left(\sum_{i=1}^{r} \mu_{i} A_{i}^{T} P\right)<0
$$

$\mathrm{Ou}$

$$
L \otimes P+M \otimes(P A(\mu))+M^{T} \otimes\left(A^{T}(\mu) P\right)<0 .
$$

$\operatorname{com} A(\mu)=\sum_{i=1}^{r} \mu_{i} A_{i}$ e $\sum_{i=1}^{r} \mu_{i}=1$.

Portanto, pela Definição 5.1 e pelo Lema 2.9, o resultado segue.

\subsection{D-Estabilidade com Funções de Lyapunov Quadráticas}

O método a seguir usa LMIs para encontrar uma matriz simétrica positiva definida comum $P$ e ganhos de realimentação $K_{i}, i=1, \ldots, r$ de forma a posicionar 
os pólos dos $r$ subsistemas locais na região desejada no plano-s. Resolvendo simultaneamente LMIs de estabilidade e de restrições de posicionamento de pólos os ganhos de realimentação $K_{i}^{\prime} s$ garantem estabilidade assintótica global e satisfazem os requisitos de desempenho determinados.

Para obter um método de projeto de controladores estabilizantes que aloquem os pólos de malha fechada na região desejada basta aplicar a condição de desempenho do sistema fuzzy em malha aberta (5.1) no sistema em malha fechada (3.26). Segue o resultado abaixo.

Teorema 5.3 O controlador fuzzy (3.25) que garante a $\mathcal{D}$-estabilidade do sistema fuzzy em malha fechada (3.26) pode ser obtido resolvendo

$$
\begin{aligned}
P & >0 \\
L \otimes P+M \otimes\left(A_{i} P+B_{i} Q_{i}\right)+M^{T} \otimes\left(P A_{i}^{T}+Q_{i}^{T} B_{i}^{T}\right) & <0, \\
2 L \otimes P+M \otimes\left(A_{i} P+A_{j} P+B_{i} Q_{j}+B_{j} Q_{i}\right) & \\
+M^{T} \otimes\left(P A_{i}^{T}+P A_{j}^{T}+Q_{i}^{T} B_{j}^{T}+Q_{j}^{T} B_{i}^{T}\right) & <0, \\
i=1,2, \ldots, r, \quad i<j \leq r, \text { sujeito } a \mu_{i} \bigcap \mu_{j} \neq \emptyset . &
\end{aligned}
$$

Os ganhos de realimentação $K_{i}, i=1,2, \ldots, r$ podem ser determinados como segue:

$$
K_{i}=Q_{i} P^{-1}, \quad i=1,2, \ldots, r
$$

Prova: Reescrevendo a equação do sistema global fuzzy em malha fechada como (3.27) e usando a forma dual de (5.1), do Lema 5.2, chega-se à condição (5.2).

Como visto na Seção 4.5.1, o sistema global fuzzy é reescrito para trabalhar com funções de Lyapunov por partes. Abaixo, seguem os resultados de análise e síntese com alocação de pólos para o sistema global fuzzy (4.30), com função de Lyapunov quadrática, antes da abordagem com funções de Lyapunov por partes.

Lema 5.4 O sistema global fuzzy (4.30) éDD-estável se existir uma matriz simétrica positiva definida tal que

$$
L \otimes P+M \otimes\left(A_{c i}(\mu) P\right)+M^{T} \otimes\left(A_{c i}(\mu) P\right)^{T}<0
$$


com $A_{c i}(\mu)=A_{i}+\Delta A_{i}(\mu)+\left(B_{i}+\Delta B_{i}(\mu)\right) K_{i}, i=1,2, \ldots, r$, e $K_{i}$ o controlador por partes (4.29).

Prova: Segue da aplicação do Teorema 2.10 no sistema em malha fechada (4.30) utilizando o mesmo procedimento de prova do Lema 5.2.

A dificuldade em resolver diretamente (5.3) está nas incertezas $\Delta A_{i}(\mu)$ e $\Delta B_{i}(\mu)$ que não são conhecidas. Para contornar esta dificuldade utiliza-se, nos próximos resultados, seus limitantes superiores (4.18).

Utilizando a definição de regiões LMIs e (5.1) tem-se o seguinte resultado de $\mathcal{D}$-estabilidade:

Teorema 5.5 O sistema global fuzzy (4.17) é D-estável se existirem constantes positivas $\epsilon_{i}, i=1,2, \ldots, r$, e uma matriz simétrica positiva definida $P$ satisfazendo as seguinte LMIs

$$
\left[\begin{array}{cc}
\phi_{i} & \epsilon_{i} I_{w} \otimes P \\
\epsilon_{i} I_{w} \otimes P & -I_{w n}
\end{array}\right]<0
$$

onde $\phi_{i}=\epsilon_{i} L \otimes P+\epsilon_{i} M \otimes\left(A_{i} P\right)+\epsilon_{i} M^{T} \otimes\left(A_{i} P\right)^{T}+\left(M M^{T}\right) \otimes\left(E_{i A} E_{i A}^{T}\right)$, $i=1,2, \ldots, r$.

Prova: Utilizando (2.18) tem-se que o sistema (4.17) é $\mathcal{D}$-estável se existir uma matriz simétrica positiva definida $P$ tal que

$$
L \otimes P+M \otimes\left(\left(A_{i}+\Delta A_{i}(\mu)\right) P\right)+M^{T} \otimes\left(\left(A_{i}+\Delta A_{i}(\mu)\right) P\right)^{T}<0 .
$$

A desigualdade (5.5) pode ser reescrita como

$$
\begin{aligned}
L \otimes P+M \otimes\left(A_{i} P\right)+ & M^{T} \otimes\left(A_{i} P\right)^{T} \\
& +M \otimes\left(\Delta A_{i}(\mu) P\right)+M^{T} \otimes\left(\Delta A_{i}(\mu) P\right)^{T}<0
\end{aligned}
$$

Aplicando a propriedade do produto de Kronecker

$$
(X \otimes Y)(U \otimes V)=X U \otimes Y V
$$


se os produtos $X U$ e $Y V$ existirem, tem-se

$$
\begin{aligned}
L \otimes P+ & M \otimes\left(A_{i} P\right)+M^{T} \otimes\left(A_{i} P\right)^{T} \\
& +\left(M \otimes \Delta A_{i}(\mu)\right)\left(I_{w} \otimes P\right)+\left(I_{w} \otimes P\right)\left(M \otimes \Delta A_{i}(\mu)\right)^{T}<0
\end{aligned}
$$

E utilizando (4.25) tem-se

$$
\begin{gathered}
L \otimes P+M \otimes\left(A_{i} P\right)+M^{T} \otimes\left(A_{i} P\right)^{T} \\
+\left(M \otimes \Delta A_{i}(\mu)\right)\left(I_{w} \otimes P\right)+\left(I_{w} \otimes P\right)\left(M \otimes \Delta A_{i}(\mu)\right)^{T} \\
\leq L \otimes P+M \otimes\left(A_{i} P\right)+M^{T} \otimes\left(A_{i} P\right)^{T} \\
\quad+\epsilon_{i}\left(I_{w} \otimes P\right)\left(I_{w} \otimes P\right)+\frac{1}{\epsilon_{i}}\left(M \otimes \Delta A_{i}(\mu)\right)\left(M \otimes \Delta A_{i}(\mu)\right)^{T} \\
=L \otimes P+M \otimes\left(A_{i} P\right)+M^{T} \otimes\left(A_{i} P\right)^{T} \\
\quad+\epsilon_{i}\left(I_{w} \otimes P\right)\left(I_{w} \otimes P\right)+\frac{1}{\epsilon_{i}}\left(M M^{T}\right) \otimes\left(\Delta A_{i}(\mu) \Delta A_{i}^{T}(\mu)\right) \\
\leq L \otimes P+M \otimes\left(A_{i} P\right)+M^{T} \otimes\left(A_{i} P\right)^{T} \\
\quad+\epsilon_{i}\left(I_{w} \otimes P\right)\left(I_{w} \otimes P\right)+\frac{1}{\epsilon_{i}}\left(M M^{T}\right) \otimes\left(E_{i A} E_{i A}^{T}\right)<0 .
\end{gathered}
$$

Aplicando o complemento de Schur em (5.7) chega-se a (5.4).

O Teorema 5.5 fornece condições para verificar se os pólos do sistema estão posicionados na região especificada pelas matrizes $L$ e $M$. Caso o sistema não possua os pólos na região desejada faz-se necessário o projeto de um controlador que leve os pólos de malha fechada até região definida no plano-s. O projeto do controlador fuzzy por partes é realizado para cada partição do espaço de estado $\overline{\mathcal{S}}_{i}, i=1,2, \ldots, r$. Utiliza-se a lei de controle de realimentação de estado e o conceito de $\mathcal{D}$-estabilidade.

Teorema 5.6 O sistema global fuzzy (4.30) é D-estável se existirem constantes positivas $\epsilon_{i}>0, i=1,2, \ldots, r$, uma matriz simétrica positiva definida $P$, e um conjunto de matrizes $\left(Q_{1}, Q_{2}, \ldots, Q_{r}\right)$ satisfazendo as LMIs

$$
\left[\begin{array}{ccc}
\phi_{i} & \epsilon_{i} I_{w} \otimes P & \epsilon_{i} I_{w} \otimes Q_{i}^{T} \\
\epsilon_{i} I_{w} \otimes P & -I_{w n} & 0 \\
\epsilon_{i} I_{w} \otimes Q_{i} & 0 & -I_{w p}
\end{array}\right]<0
$$


onde

$$
\begin{aligned}
\phi_{i} & =\epsilon_{i} L \otimes P+\epsilon_{i} M \otimes\left(A_{i} P\right)+\epsilon_{i} M^{T} \otimes\left(A_{i} P\right)^{T}+\epsilon_{i} M \otimes\left(B_{i} Q_{i}\right) \\
& +\epsilon_{i} M^{T} \otimes\left(B_{i} Q_{i}\right)^{T}+\left(M M^{T}\right) \otimes\left(E_{i A} E_{i A}^{T}\right)+\left(M M^{T}\right) \otimes\left(E_{i B} E_{i B}^{T}\right),
\end{aligned}
$$

$i=1,2, \ldots, r$,

e o ganho do controlador para cada subsistema local é dado por

$$
K_{i}=Q_{i} P^{-1}
$$

Prova: Segue diretamente do Lema 5.4 e do procedimento da prova do Teorema 5.5.

\subsection{D-Estabilidade com Funções de Lyapunov por Partes}

Nos casos de um número grande de subsistemas locais e condições muito restritivas de localização dos pólos a busca por uma função de Lyapunov comum a todos os modelos locais pode ser bastante conservadora. Como apresentado nos capítulos anteriores o uso de funções de Lyapunov por partes na análise de estabilidade ou no projeto de controladores diminui o conservadorismo na solução das LMIs, permitindo encontrar soluções para uma classe maior de problemas. Em sistemas fuzzy T-S é necessário satisfazer um grande número de LMIs, devido aos sistemas lineares locais obtidos a partir do sistema não-linear em vários pontos de operação. O critério de desempenho de localização dos pólos de malha fechada entra como uma condição mais restritiva na solução das desigualdades matriciais.

Nesta seção é apresentado o método proposto de estabilidade de sistemas fuzzy T-S que satisfaz a restrição de alocação dos pólos de malha fechada com funções de Lyapunov por partes e mantém a continuidade nas fronteiras das partições do espaço de estado.

O resultado apresentado a seguir utiliza uma parametrização da matriz $P_{i}$ através do uso de uma matriz de continuidade (Feng, 2003). 
Lema 5.7 O sistema fuzzy Takagi-Sugeno (4.17), para $x(t) \in \cup \overline{\mathcal{S}}_{i}, i=1,2, \ldots, r$, com $u(t)=0$ é $\mathcal{D}$-estável se existir matrizes simétricas $T$ e $P_{i}>0$, satisfazendo

$$
L \otimes P_{i}+M \otimes\left(P_{i}\left(A_{i}+\Delta A_{i}(\mu)\right)\right)+M^{T} \otimes\left(P_{i}\left(A_{i}+\Delta A_{i}(\mu)\right)\right)^{T}<0,
$$
$\operatorname{com} x \in \overline{\mathcal{S}}_{i} e$

$$
P_{i}=\left(F_{i}^{T} F_{i}\right)^{-1} F_{i}^{T} T F_{i}\left(F_{i}^{T} F_{i}\right)^{-1}
$$

na qual as matrizes $L$ e $M$ são escolhidas convenientemente de forma a definir a região do plano-s no qual os pólos devem pertencer.

Prova: Da condição (5.8), tem-se que o sistema (4.17) é localmente $\mathcal{D}$-estável e portanto a região LMI definida pelo subconjunto $\mathcal{D}$ é restrita ao semi-plano complexo esquerdo. Assim,

$$
P_{i}\left(A_{i}+\Delta A_{i}(\mu)\right)^{T}+\left(A_{i}+\Delta A_{i}(\mu)\right) P_{i}<0 .
$$

Baseado na condição (5.9), (5.10) e no Teorema 4.7, tem-se que o sistema (4.17) é globalmente exponencialmente estável. Conclui-se, portanto, que o sistema (4.17), para $x(t) \in \cup \overline{\mathcal{S}}_{i}, i=1,2, \ldots, r$, é $\mathcal{D}$-estável.

A seguir estabelece-se um resultado para a síntese de controladores $\mathcal{D}$-estáveis para sistemas fuzzy T-S utilizando funções de Lyapunov por partes.

Teorema $5.8 O$ sistema (4.30), para $x(t) \in \cup \overline{\mathcal{S}}_{i}, i=1,2, \ldots, r$, é $\mathcal{D}$-estável se existirem constantes positivas $\varepsilon_{i}>0$, uma matriz simétrica $T$, e um conjunto de matrizes $\left(Q_{1}, Q_{2}, \ldots, Q_{r}\right)$ tais que

$$
\begin{aligned}
& P_{i}=\left(F_{i}^{T} F_{i}\right)^{-1} F_{i}^{T} T F_{i}\left(F_{i}^{T} F_{i}\right)^{-1} \\
& {\left[\begin{array}{ccc}
\phi_{i} & \varepsilon_{i} I_{w} \otimes P_{i} & \varepsilon_{i} I_{w} \otimes Q_{i}^{T} \\
\varepsilon_{i} I_{w} \otimes P_{i} & -I_{w n} & 0 \\
\varepsilon_{i} I_{w} \otimes Q_{i} & 0 & -I_{w p}
\end{array}\right]<0}
\end{aligned}
$$

no qual

$$
\begin{gathered}
\phi_{i}=\varepsilon_{i} L \otimes P_{i}+\varepsilon_{i} M \otimes\left(A_{i} P_{i}\right)+\varepsilon_{i} M^{T} \otimes\left(A_{i} P_{i}\right)^{T}+\varepsilon_{i} M \otimes\left(B_{i} Q_{i}\right) \\
+\varepsilon_{i} M^{T} \otimes\left(B_{i} Q_{i}\right)^{T}+\left(M M^{T}\right) \otimes\left(E_{i A} E_{i A}^{T}\right)+\left(M M^{T}\right) \otimes\left(E_{i B} E_{i B}^{T}\right), \\
x \in \overline{\mathcal{S}}_{i}, \quad i=1,2, \ldots, r,
\end{gathered}
$$


e o ganho do controlador para cada subsistema local é dado por

$$
K_{i}=Q_{i} P_{i}^{-1}, \quad x \in \overline{\mathcal{S}}_{i}, \quad i=1,2, \ldots, r
$$

Prova: A partir do Lema 5.7 tem-se que o sistema em malha fechada (4.30) é $\mathcal{D}$-estável se

$$
L \otimes P_{i}+M \otimes\left(A_{c i}(\mu) P_{i}\right)+M^{T} \otimes\left(A_{c i}(\mu) P_{i}\right)^{T}<0
$$

para $P_{i}>0, x \in \overline{\mathcal{S}}_{i}$ e $i=1,2, \ldots, r$. Substituindo (4.31) em (5.13), tem-se

$$
\begin{gathered}
L \otimes P_{i}+M \otimes\left(\left(A_{i}+\Delta A_{i}(\mu)+\left(B_{i}+\Delta B_{i}(\mu)\right) K_{i}\right) P_{i}\right) \\
+M^{T} \otimes\left(\left(A_{i}+\Delta A_{i}(\mu)+\left(B_{i}+\Delta B_{i}(\mu)\right) K_{i}\right) P_{i}\right)^{T} \\
=L \otimes P_{i}+M \otimes\left(A_{i} P_{i}\right)+M^{T} \otimes\left(A_{i} P_{i}\right)^{T}+M \otimes\left(B_{i} Q_{i}\right)+M^{T} \otimes\left(B_{i} Q_{i}\right)^{T} \\
+M \otimes\left(\Delta A_{i}(\mu) P_{i}\right)+M^{T} \otimes\left(\Delta A_{i}(\mu) P_{i}\right)^{T} \\
+M \otimes\left(\Delta B_{i}(\mu) Q_{i}\right)+M^{T} \otimes\left(\Delta B_{i}(\mu) Q_{i}\right)^{T}<0
\end{gathered}
$$

para $Q_{i}=K_{i} P_{i}$. Aplicando a propriedades do produto de Kronecker (5.6), segue que

$$
\begin{aligned}
M \otimes\left(\Delta A_{i}(\mu) P_{i}\right) & =\left(M \otimes \Delta A_{i}(\mu)\right)\left(I \otimes P_{i}\right) \\
M^{T} \otimes\left(\Delta A_{i}(\mu) P_{i}\right)^{T} & =\left(I \otimes P_{i}\right)^{T}\left(M \otimes \Delta A_{i}(\mu)\right)^{T} \\
M \otimes\left(\Delta B_{i}(\mu) Q_{i}\right) & =\left(M \otimes \Delta B_{i}(\mu)\right)\left(I \otimes Q_{i}\right) \\
M^{T} \otimes\left(\Delta B_{i}(\mu) Q_{i}\right)^{T} & =\left(I \otimes Q_{i}\right)^{T}\left(M \otimes \Delta B_{i}(\mu)\right)^{T} .
\end{aligned}
$$

Então (5.14) pode ser reescrito como

$$
\begin{aligned}
& L \otimes P_{i}+M \otimes\left(A_{i} P_{i}\right)+M^{T} \otimes\left(A_{i} P_{i}\right)^{T}+M \otimes\left(B_{i} Q_{i}\right)+M^{T} \otimes\left(B_{i} Q_{i}\right)^{T} \\
& +\left(M \otimes \Delta A_{i}(\mu)\right)\left(I \otimes P_{i}\right)+\left(I \otimes P_{i}\right)^{T}\left(M \otimes \Delta A_{i}(\mu)\right)^{T} \\
& \quad+\left(M \otimes \Delta B_{i}(\mu)\right)\left(I \otimes Q_{i}\right)+\left(I \otimes Q_{i}\right)^{T}\left(M \otimes \Delta B_{i}(\mu)\right)^{T}<0
\end{aligned}
$$


Aplicando (4.25) na desigualdade acima tem-se

$$
\begin{gathered}
L \otimes P_{i}+M \otimes\left(A_{i} P_{i}\right)+M^{T} \otimes\left(A_{i} P_{i}\right)^{T}+M \otimes\left(B_{i} Q_{i}\right)+M^{T} \otimes\left(B_{i} Q_{i}\right)^{T} \\
+\left(M \otimes \Delta A_{i}(\mu)\right)\left(I \otimes P_{i}\right)+\left(I \otimes P_{i}\right)^{T}\left(M \otimes \Delta A_{i}(\mu)\right)^{T} \\
+\left(M \otimes \Delta B_{i}(\mu)\right)\left(I \otimes Q_{i}\right)+\left(I \otimes Q_{i}\right)^{T}\left(M \otimes \Delta B_{i}(\mu)\right)^{T} \\
\leq L \otimes P_{i}+M \otimes\left(A_{i} P_{i}\right)+M^{T} \otimes\left(A_{i} P_{i}\right)^{T}+M \otimes\left(B_{i} Q_{i}\right)+M^{T} \otimes\left(B_{i} Q_{i}\right)^{T} \\
+\epsilon_{i}\left(I \otimes P_{i}\right)^{T}\left(I \otimes P_{i}\right)+\frac{1}{\epsilon_{i}}\left(M \otimes \Delta A_{i}(\mu)\right)\left(M \otimes \Delta A_{i}(\mu)\right)^{T} \\
\quad+\epsilon_{i}\left(I \otimes Q_{i}\right)^{T}\left(I \otimes Q_{i}\right)+\frac{1}{\epsilon_{i}}\left(M \otimes \Delta B_{i}(\mu)\right)\left(M \otimes \Delta B_{i}(\mu)\right)^{T} \\
\leq P_{i}+M \otimes\left(A_{i} P_{i}\right)+M^{T} \otimes\left(A_{i} P_{i}\right)^{T}+M \otimes\left(B_{i} Q_{i}\right)+M^{T} \otimes\left(B_{i} Q_{i}\right)^{T} \\
+\epsilon_{i}\left(I \otimes P_{i}\right)^{T}\left(I \otimes P_{i}\right)+\frac{1}{\epsilon_{i}}\left(M M^{T}\right) \otimes\left(E_{i A} E_{i A}^{T}\right) \\
\quad+\epsilon_{i}\left(I \otimes Q_{i}\right)^{T}\left(I \otimes Q_{i}\right)+\frac{1}{\epsilon_{i}}\left(M M^{T}\right) \otimes\left(E_{i B} E_{i B}^{T}\right)<0
\end{gathered}
$$

Multiplicando a desigualdade acima por $\epsilon_{i}$ tem-se

$$
\phi_{i}+\left(\epsilon_{i} I \otimes P_{i}\right)^{T}\left(\epsilon_{i} I \otimes P_{i}\right)+\left(\epsilon_{i} I \otimes Q_{i}\right)^{T}\left(\epsilon_{i} I \otimes Q_{i}\right)<0
$$

com $\phi_{i}$ definido em (5.12). Aplicando o complemento de Schur em (5.15) finalmente chega-se a (5.11).

Para aplicar o teorema acima utilizando o aplicativo de simulação Matlab, é necessário expandir o produto de Kronecker para caracterizar as LMIs. Para o caso de uma região $\mathcal{D}$ circular de raio $r$ e centro em $(-c, 0)$, substituindo (5.12) por

$$
L=\left[\begin{array}{cc}
-r & c \\
c & -r
\end{array}\right] \text { e } M=\left[\begin{array}{ll}
0 & 1 \\
0 & 0
\end{array}\right]
$$


tem-se

$$
\begin{aligned}
\phi_{i}= & \varepsilon_{i}\left[\begin{array}{cc}
-r P_{i} & c P_{i} \\
c P_{i} & -r P_{i}
\end{array}\right]+\varepsilon_{i}\left[\begin{array}{cc}
0 & A_{i} P_{i} \\
0 & 0
\end{array}\right]+\varepsilon_{i}\left[\begin{array}{cc}
0 & 0 \\
P_{i} A_{i}^{T} & 0
\end{array}\right]+\varepsilon_{i}\left[\begin{array}{cc}
0 & B_{i} Q_{i} \\
0 & 0
\end{array}\right] \\
& +\varepsilon_{i}\left[\begin{array}{cc}
0 & 0 \\
Q_{i}^{T} B_{i}^{T} & 0
\end{array}\right]+\left[\begin{array}{cc}
E_{i A} E_{i A}^{T} & 0 \\
0 & 0
\end{array}\right]+\left[\begin{array}{cc}
E_{i B} E_{i B}^{T} & 0 \\
0 & 0
\end{array}\right], \\
= & {\left[\begin{array}{cc}
-\varepsilon_{i} r P_{i}+E_{i A} E_{i A}^{T}+E_{i B} E_{i B}^{T} & \varepsilon_{i} c P_{i}+\varepsilon_{i} A_{i} P_{i}+\varepsilon_{i} B_{i} Q_{i} \\
\varepsilon_{i} c P_{i}+\varepsilon_{i} P_{i} A_{i}^{T}+\varepsilon_{i} Q_{i}^{T} B_{i}^{T} & -\varepsilon_{i} r P_{i}
\end{array}\right], } \\
& i=1,2, \ldots, r .
\end{aligned}
$$

Então, reescrevendo $(5.11)^{2}$,

$$
\begin{aligned}
& {\left[\begin{array}{cccccc}
-\varepsilon_{i} r P_{i}+E_{i A} E_{i A}^{T}+E_{i B} E_{i B}^{T} & \varepsilon_{i} c P_{i}+\varepsilon_{i} A_{i} P_{i}+\varepsilon_{i} B_{i} Q_{i} & \varepsilon_{i} P_{i} & 0_{n \times n} & \varepsilon_{i} Q_{i}^{T} & 0_{n \times 1} \\
\varepsilon_{i} c P_{i}+\varepsilon_{i} P_{i} A_{i}^{T}+\varepsilon_{i} Q_{i}^{T} B_{i}^{T} & -\varepsilon_{i} r P_{i} & 0_{n \times n} & \varepsilon_{i} P_{i} & 0_{n \times 1} & \varepsilon_{i} Q_{i}^{T} \\
\varepsilon_{i} P_{i} & 0_{n \times n} & -I_{n \times n} & 0_{n \times n} & 0_{n \times 1} & 0_{n \times 1} \\
0_{n \times n} & \varepsilon_{i} P_{i} & 0_{n \times n} & -I_{n \times n} & 0_{n \times 1} & 0_{n \times 1} \\
\varepsilon_{i} Q_{i} & 0_{1 \times n} & 0_{1 \times n} & 0_{1 \times n} & -I_{1 \times 1} & 0_{1 \times 1} \\
0_{1 \times n} & \varepsilon_{i} Q_{i} & 0_{1 \times n} & 0_{1 \times n} & 0_{1 \times 1} & -I_{1 \times 1}
\end{array}\right]<0,} \\
& {\left[\begin{array}{cccccc}
-\varepsilon_{i} r P_{i}+E_{i A} E_{i A}^{T}+E_{i B} E_{i B}^{T} & * & * & * & * & * \\
\varepsilon_{i} c P_{i}+\varepsilon_{i} P_{i} A_{i}^{T}+\varepsilon_{i} Q_{i}^{T} B_{i}^{T} & -\varepsilon_{i} r P_{i} & * & * & * & * \\
\varepsilon_{i} P_{i} & 0_{n \times n} & -I_{n \times n} & * & * & * \\
0_{n \times n} & \varepsilon_{i} P_{i} & 0_{n \times n} & -I_{n \times n} & * & * \\
\varepsilon_{i} Q_{i} & 0_{1 \times n} & 0_{1 \times n} & 0_{1 \times n} & -I_{1 \times 1} & * \\
0_{1 \times n} & \varepsilon_{i} Q_{i} & 0_{1 \times n} & 0_{1 \times n} & 0_{1 \times 1} & -I_{1 \times 1}
\end{array}\right]<0,} \\
& i=1,2, \ldots, r .
\end{aligned}
$$

Assim, para solucionar a desigualdade (5.11), para uma região circular, equivale a resolver a LMI acima, que pode ser facilmente implementada para todos os modelos locais fuzzy $i=1, \ldots, r$.

${ }^{2} \mathrm{O}$ símbolo $*$ denota o transposto do bloco diagonalmente oposto. 


\subsection{Resultados de Simulação}

Para ilustrar a aplicação do Teorema 5.8, considera-se o problema de controle de um sistema composto de uma bola em equilíbrio em uma trave, também conhecido na literatura como Ball and Beam System, conforme Figura 5.1. As simulações foram realizadas no aplicativo Matlab. Para resolver as LMIs utilizou-se os comandos setlmis([ ]), lmivar(), lmiterm(), getlmis, feasp() e $\operatorname{dec} 2 \operatorname{mat}()$, do aplicativo LMI Control Toolbox (Gahinet et al., 1995). As simulações do sistema não-linear com os controladores obtidos a partir da resolução das LMIs de síntese foram implementadas e realizadas no módulo Simulink do Matlab, em forma de diagrama de blocos. Para implementar o controle fuzzy utiliza-se o seguinte procedimento de projeto:

1. Implementar as equações diferenciais do sistema não-linear, em forma de diagrama de blocos, para simular o sistema em malha aberta e verificar sua estabilidade;

2. Obter os modelos lineares nos pontos de linearização escolhidos;

3. Elaborar as regras de modelo fuzzy e das funções de pertinência para a construção do modelo fuzzy Takagi-Sugeno;

4. Especificar os critérios de desempenho desejados - região do plano-s da localização dos pólos de malha fechada - e parâmetros de projeto - $L, M$, $\epsilon_{i}$ e limitantes $E_{i A}$ e $E_{i B}, i=1 \ldots, r$;

5. Resolver as LMIs para um conjunto de matrizes positiva definidas $P_{i}$ e matrizes $Q_{i}, i=1 \ldots, r$. Este passo pode ser facilmente implementado com o uso do aplicativo LMI Control Toolbox (Gahinet et al., 1995);

6. Se as LMIs forem factíveis, os controladores podem ser obtidos por $K_{i}=$ $Q_{i} P_{i}^{-1}, i=1 \ldots, r$. Caso contrário, redefinir $\epsilon_{i}=\epsilon_{i} / 2, i=1 \ldots, r$, e voltar ao passo anterior. Verificar se $\epsilon_{i}$ é maior do que um limitante definido, caso contrário declarar o projeto do controlador sem solução. 
7. Implementar os controladores obtidos no diagrama do Passo 1;

8. Verificar a estabilidade e desempenho do sistema em malha fechada através de simulações para diversas condições iniciais.

\subsubsection{Projeto do controlador}

Suponha que a bola tem apenas um grau liberdade, ou seja, movimenta-se em apenas uma dimensão. A trave é feita para rotacionar no plano vertical pela aplicação de uma força de torque aplicado ao centro da trave e a bola é livre para rolar ao longo dela. Esta deve se manter em contato com a trave e não deve haver escorregamento da bola com a trave.

As equações dinâmicas do sistema são descritas a seguir (Feng, 2003):

$$
\begin{aligned}
\left(J_{b} R^{2}+M\right) \ddot{\psi}+M g \operatorname{sen} \theta-M \psi \dot{\theta}^{2} & =0 \\
\left(M \psi^{2}+J+J_{b}\right) \ddot{\theta}+2 M \psi \dot{\psi} \dot{\theta}+M g \psi \cos \theta & =\tau,
\end{aligned}
$$

onde $\psi$ é a posição da bola, $\theta$ é o ângulo da trave, $g=9,8 \mathrm{~m} / \mathrm{s}^{2}$ é a constante gravitacional, $J$ é o momento de inércia da trave, $M, J_{b}$, e $R$ são a massa, o momento de inércia e o raio da bola respectivamente, e $\tau$ é o torque aplicado a trave.

Definindo $b:=M /\left(J_{b} / R^{2}+M\right)$ e fazendo

$$
\tau=2 M \psi \psi \dot{\psi}+M g \psi \cos \theta+\left(M \psi^{2}+J+J_{b}\right) u
$$

onde $u$ é a nova entrada, o sistema é reescrito pela seguinte equação no espaço de estado

$$
\begin{aligned}
& \left\{\begin{array}{l}
\dot{x}_{1}=x_{2} \\
\dot{x}_{2}=b\left(x_{1} x_{4}^{2}-g \operatorname{sen}\left(x_{3}\right)\right) \\
\dot{x}_{3}=x_{4} \\
\dot{x}_{4}=u
\end{array}\right. \\
& y=x_{1},
\end{aligned}
$$

onde $x=\left[\begin{array}{llll}x_{1} & x_{2} & x_{3} & x_{4}\end{array}\right]:=\left[\begin{array}{llll}\psi & \dot{\psi} & \theta & \dot{\theta}\end{array}\right]$. Os parâmetros do sistema são escolhidos como em (Ma e Sun, 2000), ou seja, $M=0,05 K g, R=0,01 \mathrm{~m}, J=0,02 \mathrm{Kgm}^{2}$, $J_{b}=2 \times 10^{-6} \mathrm{kgm}^{2}$, e $g=9,98 \mathrm{~m} / \mathrm{s}^{2}$. Então, $b=0,7143$. 


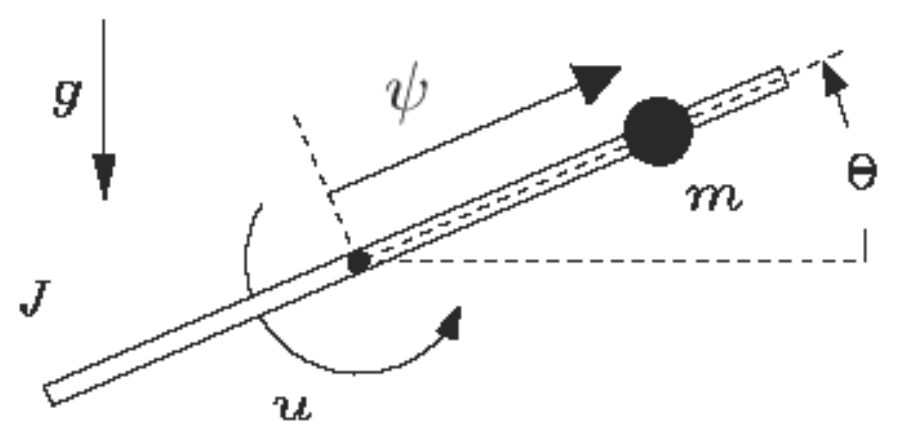

Figura 5.1: Sistema com uma bola em equilíbrio em uma trave.

O sistema não-linear em malha aberta pode ser visto nas Figuras 5.3 e 5.4.

O sistema bola-trave é um exemplo clássico na aplicação de técnicas de controle. O termo crítico $x_{1} x_{4}^{2}$ aparece na equação de $\dot{x}_{2}$. Este termo cresce quadraticamente com a velocidade angular da trave $x_{4}=\dot{\theta}$. Através da análise do sistema físico e de simulações é possível concluir que é suficiente a trave estar perfeitamente horizontal $\left(x_{3}=0\right.$ e $\left.x_{4}=0\right)$ e a bola em total repouso $\left(x_{2}=0\right)$ para que esta se mantenha em sua posição inicial, porém qualquer pertubação leva o sistema à instabilidade. Para condições iniciais diferentes de zero o sistema tende a instabilidade.

O objetivo do controle é determinar $u(x)$ tal que a saída em malha fechada converge para zero para certas condições iniciais.

Para a implementação do controlador será projetado os seguintes casos:

1. Controlador fuzzy via CDP com realimentação de estado - utilizando (3.30);

2. Controlador fuzzy via CDP com realimentação de estado e restrição no posicionamento dos pólos - utilizando Teorema 5.3;

3. Controlador chaveado fuzzy com realimentação de estado e restrição no posicionamento dos pólos através de funções de Lyapunov por partes - utilizando o Teorema 5.8 .

Para aplicar o controle fuzzy é necessário linearizar o sistema original em pontos escolhidos. Escolhem-se os pontos $\bar{x}_{A}=(-1,0,0,0.01)$ e $\bar{x}_{B}=(1,0,0,0.01)$. 
Os pontos escolhidos representam duas posições distintas da trave a uma pequena velocidade angular. Através da técnica de linearização convencional (3.7) não é possível obter modelos lineares devido a presença de termos constantes. Para contornar este problema será utilizado a metodologia proposta por Teixeira e Zak (1999), apresentada na Seção 3.1.2.

Aplicando (3.23) para $\bar{x}_{A}$, tem-se

$$
\begin{aligned}
& a_{1}^{T}=\left[\begin{array}{llll}
0 & 1 & 0 & 0
\end{array}\right], \\
& a_{2}^{T}=\left[\begin{array}{llll}
0 & 0 & -b g & 2 b \beta
\end{array}\right] \\
& a_{3}^{T}=\left[\begin{array}{llll}
0 & 0 & 0 & 1
\end{array}\right], \\
& a_{4}^{T}=\left[\begin{array}{llll}
0 & 0 & 0 & 0
\end{array}\right],
\end{aligned}
$$

onde $\beta=0.01$ e $a_{i}^{T}, i=1,2$, é a i-ésima linha de $A_{1}$. Portanto,

$$
A_{1}=\left[\begin{array}{cccc}
0 & 1 & 0 & 0 \\
0 & 0 & -b g & -2 b \beta \\
0 & 0 & 0 & 1 \\
0 & 0 & 0 & 0
\end{array}\right]
$$

A matriz $B$ é obtida a partir de (3.9).

$$
B_{1}=\left[\begin{array}{l}
0 \\
0 \\
0 \\
1
\end{array}\right]
$$

Aplicando o mesmo procedimento para $\bar{x}_{B}$, tem-se

$$
A_{2}=\left[\begin{array}{cccc}
0 & 1 & 0 & 0 \\
0 & 0 & -b g & 2 b \beta \\
0 & 0 & 0 & 1 \\
0 & 0 & 0 & 0
\end{array}\right]
$$

e $B_{2}=B_{1}$.

A partir dos sistemas linearizados acima as seguintes regras fuzzy são construídas para descrever o sistema não-linear: 


\section{Regra do Modelo 1:}

$\mathrm{SE}$

$$
x_{1}(t) \text { está em torno }-1 \text {, }
$$

ENTÃO $\quad \dot{x}(t)=A_{1} x(t)+B_{1}(t) u(t)$

\section{Regra do Modelo 2:}

$\mathrm{SE}$

$$
x_{1}(t) \text { está em torno } 1 \text {, }
$$

ENTÃO $\quad \dot{x}(t)=A_{2} x(t)+B_{2}(t) u(t)$

São usadas funções de pertinência trapezoidais para descrever a pertinência das funções de acordo com a variável premissa, o estado $x_{1}$, como mostra a Figura 5.2. Escolhe-se a região de círculo de centro em $(-5,0)$ e raio $r=2.5$ no

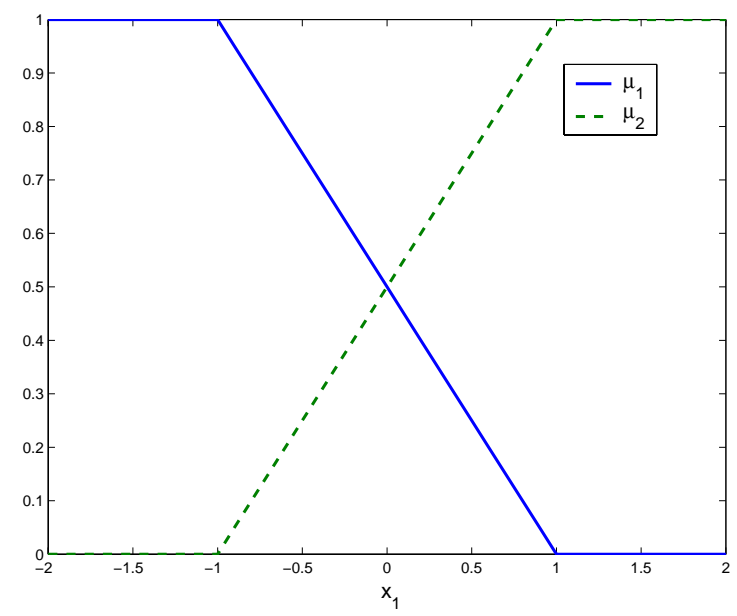

Figura 5.2: Funções de pertinência fuzzy.

plano-s para a qual os pólos do sistema em malha fechada deverão pertencer para caracterizar o desempenho desejado.

As seguintes matrizes descrevem a região LMI desejada, segundo a Definição 2.8:

$$
L=\left[\begin{array}{cc}
-2.5 & 5 \\
5 & -2.5
\end{array}\right] \text { e } M=\left[\begin{array}{ll}
0 & 1 \\
0 & 0
\end{array}\right]
$$

Para a síntese dos controladores via CDP a seguinte lei de realimentação de estado é utilizada:

$$
u(t)=\sum_{i=1}^{r} \mu_{i}(x(t)) K_{i} x(t) .
$$


Caso 1: Este caso apresentou estabilidade, porém os pólos do sistema em malha fechada não se encontram na região de círculo de centro em $(-5,0)$ e raio $r=2.5$ no plano-s, conforme mostra a Figura 5.9. Os seguintes ganhos locais dos subsistemas lineares foram obtidos

$$
\begin{aligned}
& K_{1}=\left[\begin{array}{llll}
6.32 & 6.03 & -42.51 & -6.096
\end{array}\right] \\
& K_{2}=\left[\begin{array}{llll}
6.25 & 5.90 & -42.24 & -6.029
\end{array}\right]
\end{aligned}
$$

A resposta à condição inicial $x_{0}=\left[\begin{array}{llll}1 & 0 & 0 & 0\end{array}\right]^{\prime}$ é mostrada nas Figuras 5.12 e 5.13.

Caso 2: Utilizando a técnica do Teorema 5.3, para construir um controlador fuzzy via CDP, que posicione os pólos na região especificada por $L$ e $M$, definidos acima, obteve-se os seguintes ganhos que resultaram no posicionamento satisfatório dos pólos de malha fechada:

$$
\begin{aligned}
& K_{1}=\left[\begin{array}{llll}
38.39 & 37.90 & -99.86 & -16.43
\end{array}\right] \\
& K_{2}=\left[\begin{array}{llll}
37.94 & 37.51 & -99.07 & -16.22
\end{array}\right]
\end{aligned}
$$

O controlador fuzzy obtido foi implementado no sistema não-linear original (5.16). Utilizou-se como condição inicial $x_{0}=\left[\begin{array}{llll}1 & 0 & 0 & 0\end{array}\right]^{\prime}$. Através de diversas simulações observou-se que o controlador era capaz de responder no intervalo $x_{10} \in\left[\begin{array}{ll}-1.4 & 1.4\end{array}\right]\left(x_{20}, x_{30}, x_{40}=0\right)$, como mostram as Figuras 5.12 e 5.13.

Caso 3: A seguir, é implementado a síntese de controle proposta. Deseja-se encontrar funções de Lyapunov por partes que atendam aos requisitos de projeto. Para tal, faz-se necessário a delimitação de regiões no espaço de estado $\overline{\mathcal{S}}_{1}=\{x$ : $\left.x_{1}<0\right\}$ e $\overline{\mathcal{S}}_{2}=\left\{x: x_{1}>0\right\}$. No sistema real em malha fechada o estado $x_{1}$ define a comutação dos controladores $K_{1}$ e $K_{2}$ nas regiões $\overline{\mathcal{S}}_{1}$ e $\overline{\mathcal{S}}_{2}$.

Para garantir a continuidade da função de Lyapunov segundo o Lema 4.4, define-se as funções de continuidade $F_{1}$ e $F_{2}$ para as regiões $x_{1} \in \overline{\mathcal{S}}_{1}$ e $x_{1} \in \overline{\mathcal{S}}_{2}$ respectivamente. A metodologia para encontrar tais funções está descrito em 
Johansson et al. (1999).

$$
F_{1}=\left[\begin{array}{cccc}
-1 & 0 & 0 & 0 \\
1 & 0 & 0 & 0 \\
0 & 1 & 0 & 0 \\
0 & 0 & 1 & 0 \\
0 & 0 & 0 & 1
\end{array}\right] \text { e } F_{2}=\left[\begin{array}{cccc}
1 & 0 & 0 & 0 \\
1 & 0 & 0 & 0 \\
0 & 1 & 0 & 0 \\
0 & 0 & 1 & 0 \\
0 & 0 & 0 & 1
\end{array}\right]
$$

Os seguintes limitantes superiores foram encontrados utilizando (4.19):

$$
E_{i A}=\sum_{j \in \mathcal{H}(i)} \mu_{j}(\bar{x}) \Delta A_{i j}
$$

O pior caso, ou seja, $E_{i A}$ é máximo, ocorre quando $\bar{x}$ é o estado tal que $\mu_{i}(\bar{x})=$ 0,5. Então,

$$
\begin{aligned}
& E_{1 A}=\mu_{2}(\bar{x}) \Delta A_{12}=0,5(A 2-A 1), \\
& E_{2 A}=E_{1 A}=\left[\begin{array}{cccc}
0 & 0 & 0 & 0 \\
0 & 0 & 0 & -0.01429 \\
0 & 0 & 0 & 0 \\
0 & 0 & 0 & 0
\end{array}\right] .
\end{aligned}
$$

De forma análoga,

$$
E_{1 B}=E_{2 B}=0,5(B 2-B 1)=\left[\begin{array}{l}
0 \\
0 \\
0 \\
0
\end{array}\right] .
$$

A factibilidade da LMI foi obtida com $\epsilon_{1}=\epsilon_{2}=1,0 \times 10^{-1}$ e uma matriz comum $T$, matrizes positivas definidas $P_{1}$ e $P_{2}$ e matrizes $Q_{1}$ e $Q_{2}$ foram encon- 
tradas:

$$
\begin{aligned}
T= & {\left[\begin{array}{rrrrr}
-23700 & -0.000763 & 0.00136 & 0.000688 & -0.00247 \\
-0.000763 & 23700 & -0.120 & -0.0475 & 0.128 \\
0.00136 & -0.120 & 0.192 & 0.0847 & -0.262 \\
0.000688 & -0.0475 & 0.0847 & 0.0423 & -0.150 \\
-0.00247 & 0.128 & -0.2620 & -0.150 & 0.609
\end{array}\right], } \\
P_{1}= & {\left[\begin{array}{cccc}
0.0212 & -0.0610 & -0.0241 & 0.0654 \\
-0.0610 & 0.192 & 0.0847 & -0.262 \\
-0.0241 & 0.0847 & 0.0423 & -0.150 \\
0.0654 & -0.262 & -0.150 & 0.609
\end{array}\right] } \\
P_{2}= & {\left[\begin{array}{cccc}
0.0205 & -0.0596 & -0.0234 & 0.0629 \\
-0.0596 & 0.192 & 0.0847 & -0.262 \\
-0.0234 & 0.0847 & 0.0423 & -0.150 \\
0.0629 & -0.262 & -0.150 & 0.609
\end{array}\right] } \\
Q_{1}= & {\left[\begin{array}{llll}
-0.172 & 0.805 & 0.531 & -2.47
\end{array}\right] } \\
Q_{2}= & {\left[\begin{array}{llll}
-0.159 & 0.790 & 0.522 & -2.43
\end{array}\right] }
\end{aligned}
$$

e os respectivos controladores

$$
\begin{aligned}
& K_{1}=\left[\begin{array}{llll}
29.03 & 30.62 & -85.76 & -15.12
\end{array}\right] \\
& K_{2}=\left[\begin{array}{llll}
27.63 & 29.23 & -82.96 & -14.70
\end{array}\right]
\end{aligned}
$$

Através da lei de controle $u(t)=K_{i} x(t), x(t) \in \overline{\mathcal{S}}_{i}$, os pólos de malha fechada foram alocadas na região desejada conforme mostra a Figura 5.11. Através das simulações foi possível constatar que o sistema consegue alcançar o equilíbrio e resposta temporal satisfatória para a condição inicial $x_{0}=\left[\begin{array}{llll}1 & 0 & 0 & 0\end{array}\right]^{\prime}$ (ver Figuras 5.12 e 5.13$)$.

Observação 5.1 Observa-se que a técnica proposta utilizando funções de Lyapunov por partes (caso 3) apresenta uma resposta mais rápida em relação ao caso 1, e resultados similares em relação ao uso da P comum (caso 2), porém, a factibilidade das LMIs pode ser mais facilmente atingida com funções de Lyapunov 
por partes no caso de modelos fuzzy serem constítuidos de um número maior de modelos locais. 


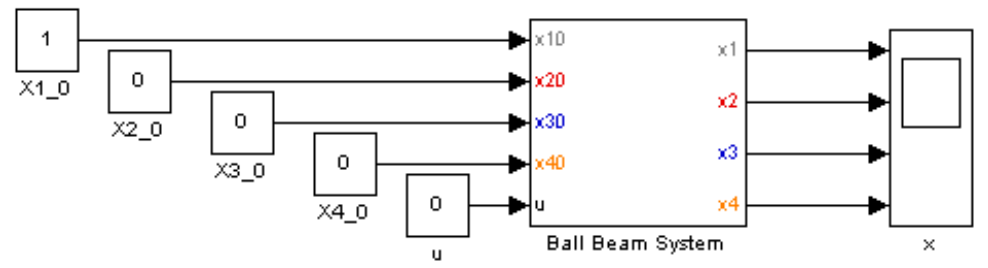

Figura 5.3: Diagrama Simulink do sistema não-linear em malha aberta.

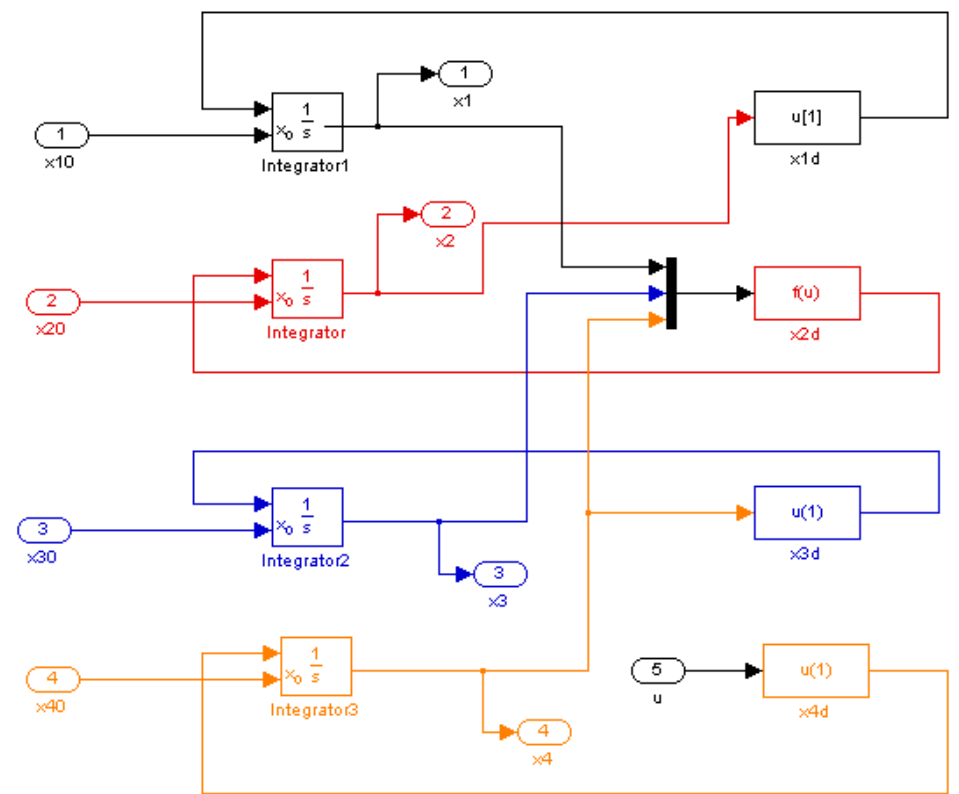

Figura 5.4: Diagrama Simulink do sistema Bola-Trave.

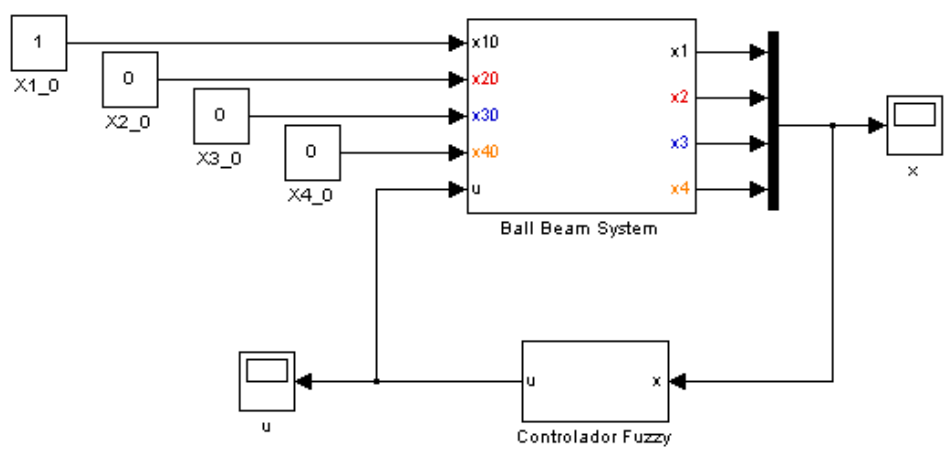

Figura 5.5: Diagrama Simulink do sistema em malha fechada com o controlador fuzzy via CDP. 


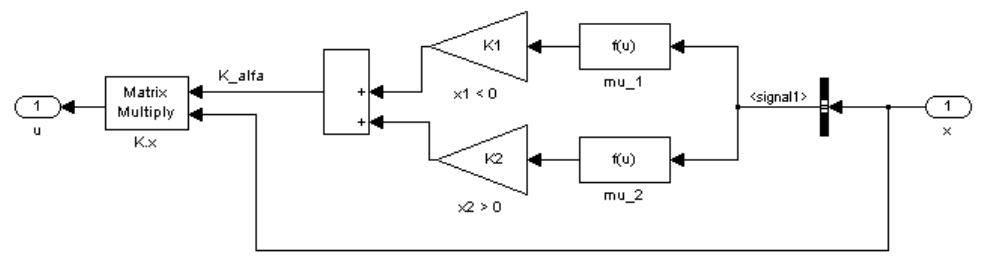

Figura 5.6: Diagrama Simulink da estrutura interna do controlador fuzzy via CDP.

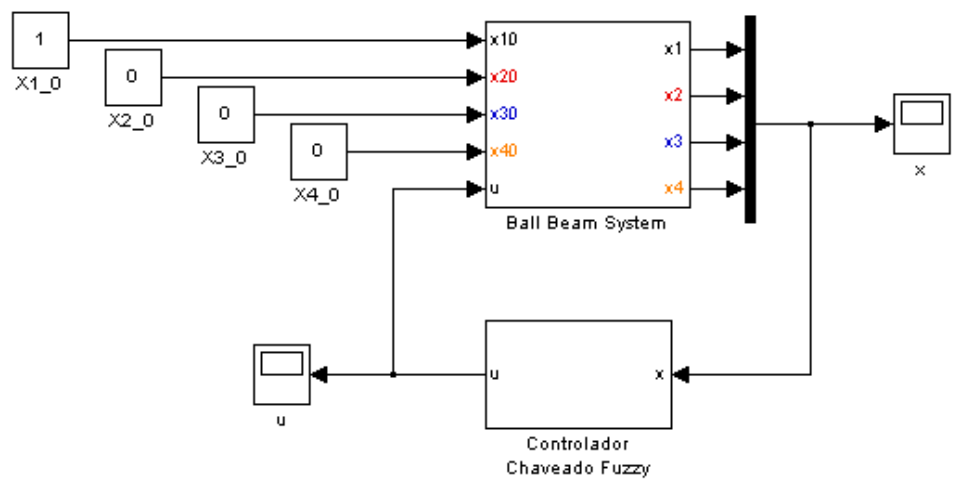

Figura 5.7: Diagrama Simulink do sistema em malha fechada com o controlador chaveado fuzzy.

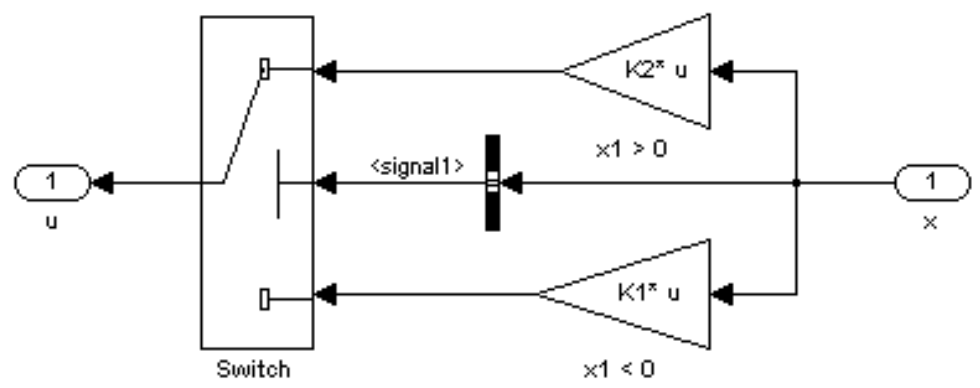

Figura 5.8: Estrutura do controlador chaveado fuzzy. 


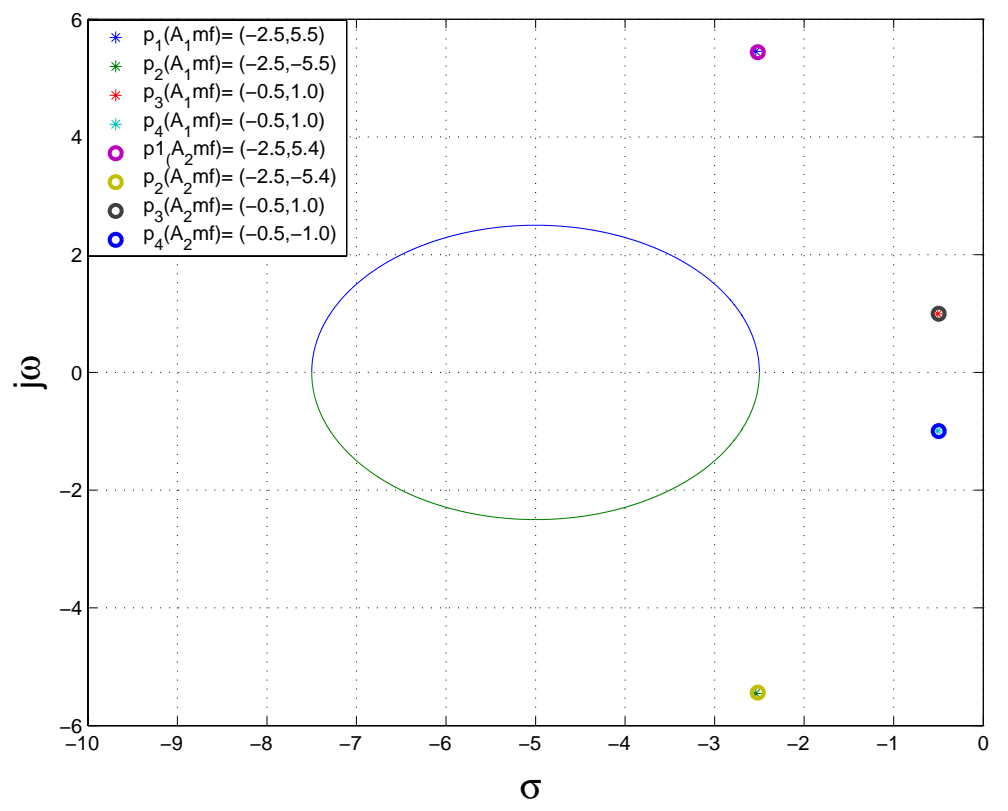

Figura 5.9: Pólos de malha fechada dos subsistemas lineares do caso 1.

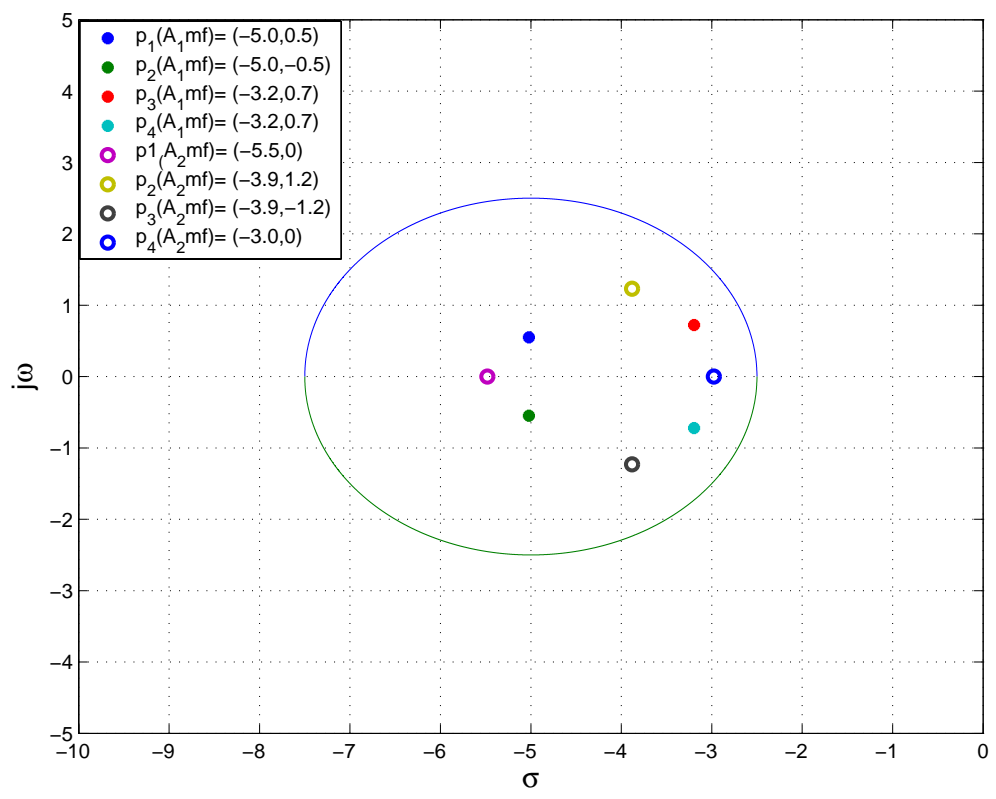

Figura 5.10: Pólos de malha fechada dos subsistemas lineares locais do caso 2. 


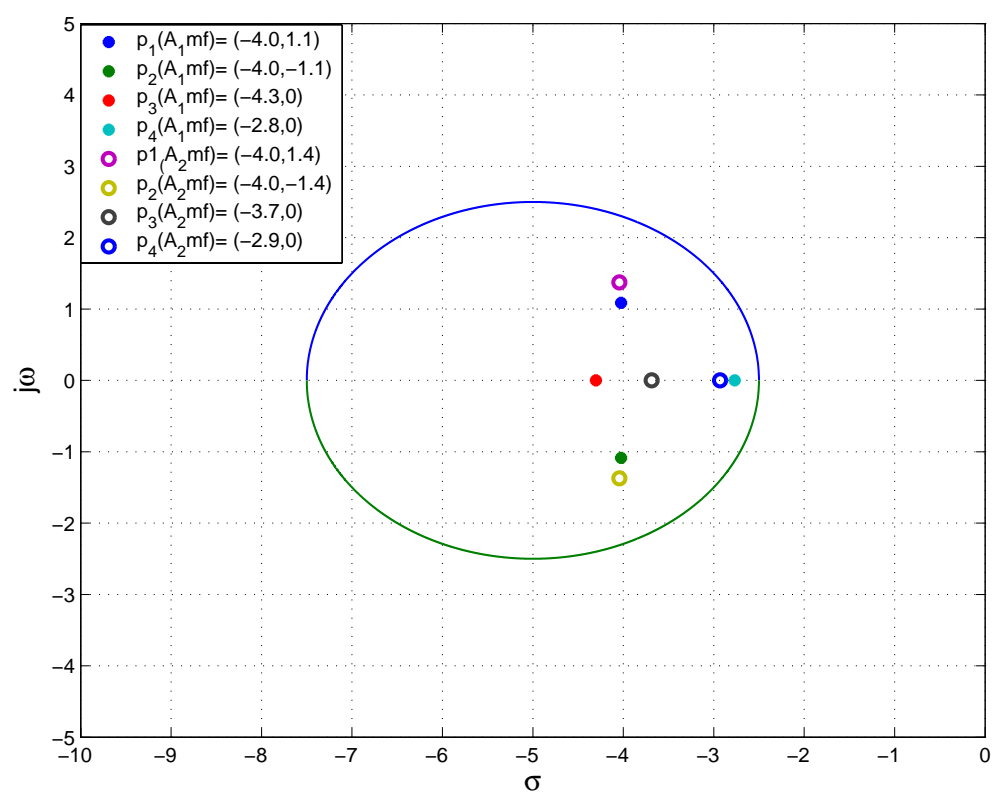

Figura 5.11: Localização dos pólos dos subsistemas de malha fechada do caso 3. 

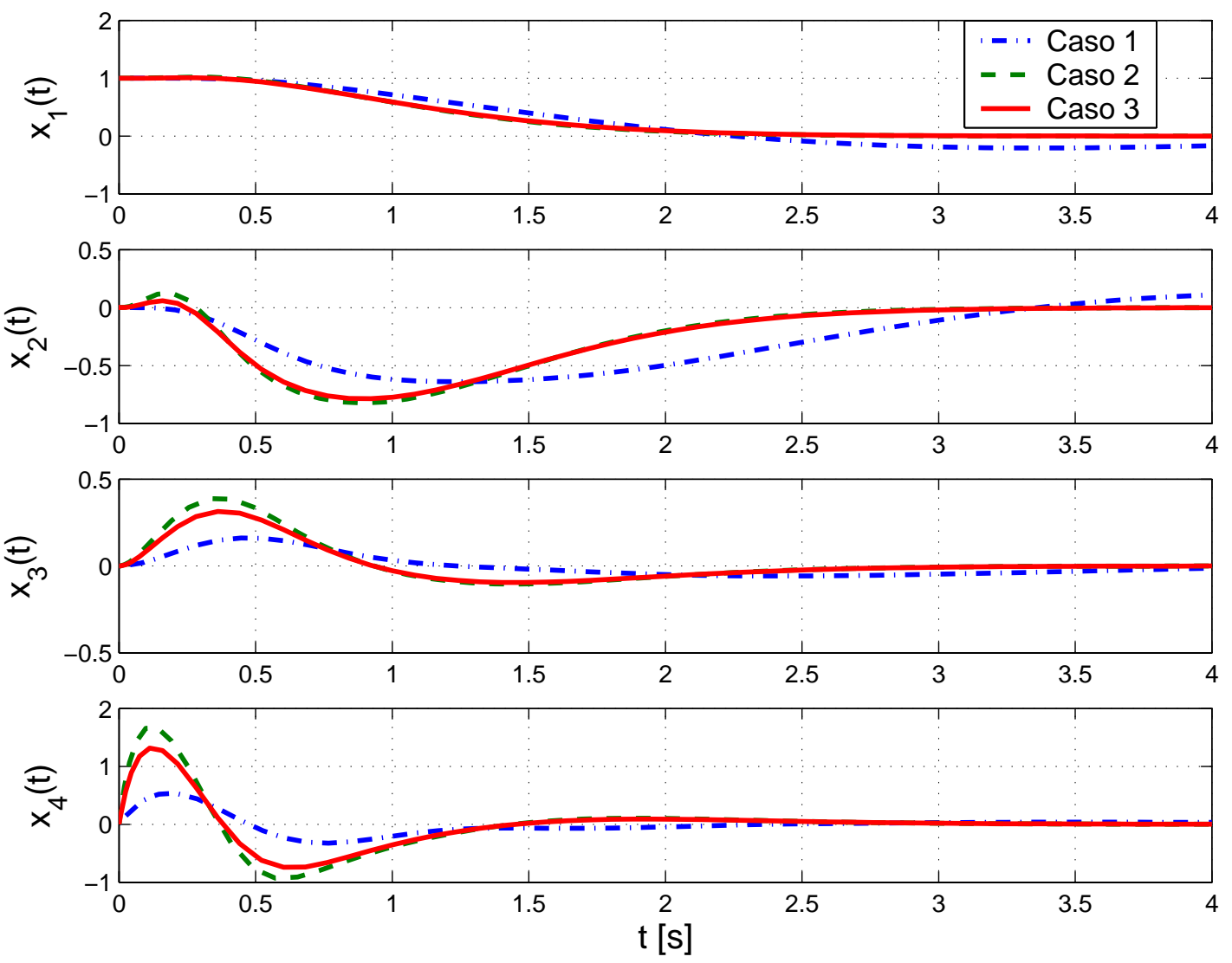

Figura 5.12: Resposta do sistema em malha fechada para os casos 1, 2 e 3 com condição inicial [1 $\left.\begin{array}{llll}1 & 0 & 0 & 0\end{array}\right]$. 


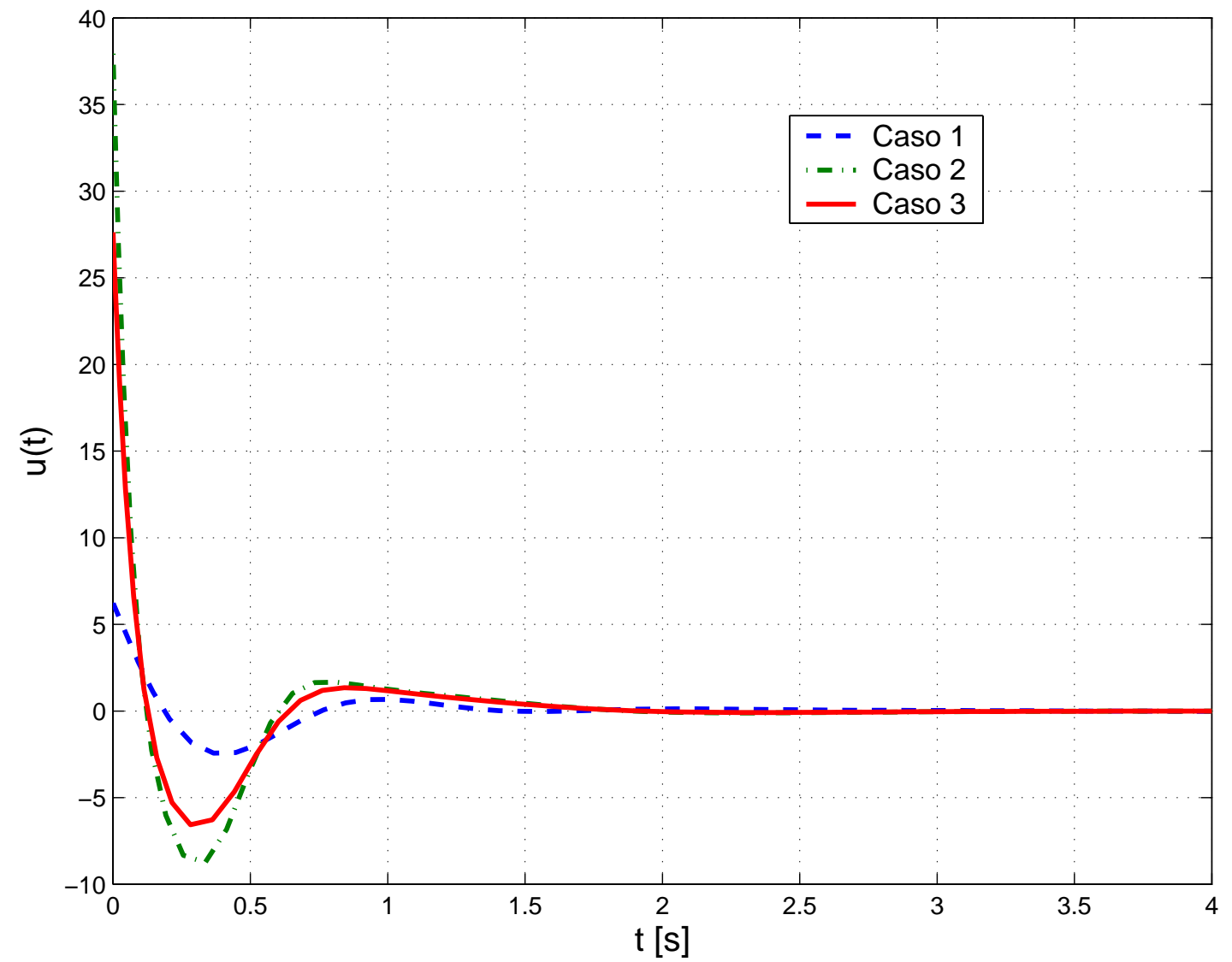

Figura 5.13: Resposta do sinal de controle para os casos 1, 2 e 3 com condição inicial $\left[\begin{array}{llll}1 & 0 & 0 & 0\end{array}\right]$. 


\section{Capítulo 6}

\section{Conclusão e Proposta para Trabalhos Futuros}

\subsection{Conclusão}

A modelagem fuzzy Takagi-Sugeno é adequada para a análise matemática de sistemas dinâmicos e permite a aplicação de técnicas desenvolvidas para sistemas lineares. Sendo assim, muitas técnicas de estabilidade e de projeto de controladores têm sido desenvolvidas para sistemas fuzzy T-S. Técnicas como realimentação de estado, realimentação de saída, construção de observadores têm sido aplicadas em conjunto com técnicas de robustez e de controle ótimo utilizando a teoria de estabilidade de Lyapunov e resolvidas através do uso de LMIs. A técnica de alocação de pólos permite especificar um comportamento desejado para a resposta transitória do sistema.

Em sistemas fuzzy existem diversos trabalhos abordando a alocação de pólos com o uso de LMIs e com rejeição à distúrbios através de controles $H_{2}$ e $H_{\infty}$. Porém, uma dificuldade existente na análise de estabilidade ou no projeto de controladores é encontrar uma função de Lyapunov que satisfaça as restrições impostas pelos requisitos de projeto. Neste sentido, alguns artigos publicados utilizam funções de Lyapunov por partes para a análise de estabilidade e síntese 
de controladores robustos. O uso de funções de Lyapunov por partes é introduzido em sistemas fuzzy por Johansson et al. (1999). Nesse trabalho também é utilizada a continuidade das funções de Lyapunov, a partir de Rantzer e Johansson (2000). Em Feng e Sun (2002) o autor utiliza as funções de Lyapunov por partes para a síntese de um controlador $H_{2}$ para sistemas fuzzy T-S. O uso destas funções no problema de alocação de pólos de sistemas fuzzy têm sido pouco abordado nas revistas especializadas.

Este trabalho propõe uma técnica de alocação de pólos para sistemas fuzzy Takagi-Sugeno utilizando funções de Lyapunov por partes e contínuas nas partições do espaço de estado via desigualdades matriciais lineares. A técnica tem como vantagem prover condições menos conservadoras em relação àquelas obtidas com o uso de funções de Lyapunov quadráticas para encontrar um controlador que aloque os pólos de malha fechada na região desejada do semi-plano esquerdo do plano-s.

\subsection{Proposta para Trabalhos Futuros}

No sentido de gerar futuras contribuições, no que segue são apresentadas algumas propostas para trabalhos futuros com base nos resultados obtidos até este ponto.

- Aplicação do método proposto para sistemas fuzzy afins;

- Desenvolvimento de condições mais relaxadas, utilizando as informações das partições, através do procedimento S;

- Combinação dos resultados com técnicas de robustez, como $H_{2}$ e $H_{\infty}$;

- Uso de realimentação de saída, através da construção de observadores;

- Extensão dos resultados para o caso discreto. 


\section{Bibliografia}

Assawinchaichote, W. e Nguang, S. K. (2004). $H_{\infty}$ filtering for fuzzy singularly perturbed systems with pole placement constraints: An LMI approach, IEEE Transactions on Signal Processing 52(6): 1659-1667.

Baumann, W. T. e Rugh, W. J. (1986). Feedback control of nonlinear systems by extended linearization, IEEE Transactions Automatic Control 31(1): 40-46.

Bontempi, G. e Birattari, M. (2005). From linearization to lazy learning: A survey of divide-and-conquer techniques for nonlinear control, International Journal of Computation Cognition 3(1): 56-73.

Boyd, S., Ghaoui, L. E., Feron, E. e Balakrishnan, V. (1994). Linear Matrix Inequalities in System and Control Theory, Society for Industrial and Applied Mathematics (SIAM), Philadelphia, PA.

Cao, S. G., Rees, N. W. e Feng, G. (1996). Fuzzy control of nonlinear continoustime systems, Proceedings of 35th IEEE Conf. Decision and Control, Kobe, Japan, pp. 592-597.

Chilali, M. e Gahinet, P. (1996). $H_{\infty}$ design with pole placement constraints: An LMI approach, IEEE Transactions on Automatic Control 41(3): 358-367.

Chilali, M., Gahinet, P. e Apkarian, P. (1999). Robust pole placement in LMI regions, IEEE Transactions on Automatic Control 44(12): 2257-2270.

Chong, E. K. P. e Zak, S. H. (1996). An Introduction to Optimization, Wiley, New York, NY. 
Feng, G. (2003). Controller synthesis of fuzzy dynamic systems based on piecewise Lyapunov functions, IEEE Transactions on Fuzzy Systems 11(5): 605-612.

Feng, G. (2004). $H_{\infty}$ controller design of fuzzy dynamic systems based on piecewise Lyapunov functions, IEEE Transactions on Systems, Man, and Cybernetics - Part B: Cybernetics 34(1): 283-292.

Feng, G., Chen, C., Sun, D. e Zhu, Y. (2005). $H_{\infty}$ controller synthesis of fuzzy dynamic systems based on piecewise Lyapunov functions and bilinear matrix inequalities, IEEE Transactions on Fuzzy Systems 13(1): 94-103.

Feng, G., Lu, G. P. e Zhou, S. S. (2002). An approach to $H_{\infty}$ controller synthesis of piecewise linear systems, Communications In Information and Systems 2(3): $245-254$.

Feng, G. e Sun, D. (2002). Generalized $H_{2}$ controller synthesis of fuzzy dynamic systems based on piecewise Lyapunov functions, IEEE Transactions on Circuits and Systems-I: Fundamental Theory And Applications 49(12): 18431850.

Gahinet, P. e Apkarian, P. (1994). A linear matrix inequality approach to $H_{\infty}$ control, International Journal of Robust and Nonlinear Control 4: 421-448.

Gahinet, P., Nemirovsky, A., Laub, A. J. e Chilali, M. (1995). LMI Control Toolbox for use with Matlab, The Mathworks Inc., Natick, MA.

Haddad, W. M. e Bernstein, D. S. (1992). Controller design with regional pole constraints, IEEE Transactions on Automatic Control 37(1): 54-69.

Han, Z. X., Feng, G., Walcott, B. L. e Zhang, Y. M. (2000). $H_{\infty}$ controller design of fuzzy dynamic systems with pole placement constraints, Proceedings of the American Control Conference, Vol. 3, Chicago, IL, pp. 1939-1943.

Hong, S. K. e Nam, Y. (2003). Stable fuzzy control system design with poleplacement constraint: an LMI approach, Computers in Industry 51(1): 1-11. 
Joh, J., Langari, R., Jeung, E. T. e Chung, W. J. (1997). A new design method for continuous Takagi-Sugeno fuzzy controller with pole placement constraints: An LMI approach, IEEE International Conference on Systems, Man and Cybernetics, Vol. 3, Orlando, FL, pp. 2969-2974.

Johansen, T. A., Shorten, R. e Murray-Smith, R. (2000). On the interpretation and identification of dynamic Takagi-Sugeno fuzzy models, IEEE Transactions on Fuzzy Systems 8(3): 297-313.

Johansson, M. (1999). Piecewise Linear Control Systems, Tese de Doutorado, Lund Institute of Technology, Sweden.

Johansson, M. e Rantzer, A. (1998). Computation of piecewise quadratic Lyapunov functions for hybrid systems, IEEE Transactions on Automatic Control 43(4): 555-559.

Johansson, M., Rantzer, A. e Arzen, K. (1999). Piecewise quadratic stability of fuzzy systems, IEEE Transactions on Fuzzy Systems 7(6): 713-722.

Khalil, H. K. (1996). Nonlinear systems, 2 ed, Prentice-Hall, Englewood Cliffs, NJ.

Liberzon, D. (2003). Switching in Systems and Control, 1 ed, Birkhauser, Boston, MA.

Ma, X. J. e Sun, Z. Q. (2000). Output tracking and regulation of nonlinear system based on Takagi-Sugeno fuzzy model, IEEE Transactions System, Man, Cybernetics B 30: 47-59.

Petersen, I. R. (1987). A stabilization algorithm for a class of uncertain linear systems, System and Control Letters 8: 351-357.

Rantzer, A. e Johansson, M. (2000). Piecewise linear quadratic optimal control, IEEE Transactions on Automatic Control 45(4): 629-637.

Rao, P. S. e Sen, I. (2000). Robust pole placement stabilizer design using linear matrix inequalities, IEEE Transactions on Power Systems 15(1): 313-319. 
Scherer, C., Gahinet, P. e Chilali, M. (1997). Multiobjective output-feedback control via LMI optimization, IEEE Transactions on Automatic Control 42(7): 896-911.

Shorten, R., Murray-Smith, R., Bjorgan, R. e Gollee, H. (1999). On the interpretation of local models in blended multiple model structures, International Journal of Control 72(7/8): 620-628.

Takagi, T. e Sugeno, M. (1985). Fuzzy identification of systems and its applications to modeling and control, IEEE Transactions on Systems, Man and Cybernetics 15(1): 116-132.

Tanaka, K. e Sugeno, M. (1992). Stability analysis and design of fuzzy control systems, Fuzzy Sets and Systems 45(2): 135-156.

Tanaka, K. e Wang, H. O. (2001). Fuzzy Control Systems Design and Analysis A Linear Matrix Inequality Approach, Wiley-Interscience Publication, New York, NY.

Teixeira, M. C. M., Daruichi, E. R. M. M. e Assunção, E. (2002). Construção de modelos fuzzy Takagi-Sugeno reduzidos para sistemas não-lineares, Congresso Brasileiro de Automática, Natal, RN, pp. 1885-1892.

Teixeira, M. C. M. e Zak, S. H. (1999). Stabilizing controller design for uncertain nonlinear systems using fuzzy models, IEEE Transactions on Fuzzy systems 7(2): 133-142.

Vandenbergue, L. e Boyd, S. (1993). A polynomial-time algorithm for determining quadratic Lyapunov functions for nonlinear systems, Proceedings of the European Conference on Circuit Theory and Design, Davos, Suiça, pp. 10651068.

Wang, H., Li, J., Niemann, D. e Tanaka, K. (2000). T-s fuzzy model with linear rule consequence and pdc controller: a universal framework for nonlinear 
control systems, The 9th IEEE International Conference on Fuzzy Systems, San Antonio, TX, Vol. 2, pp. 549-554.

Wang, H. O., Tanaka, K. e Griffin, M. F. (1995). Parallel distributed compensation of nonlinear systems by Takagi-Sugeno fuzzy model, Proc. IEEE Int. Conf. on Fuzzy Systems, Yokohama, Japan, pp. 531-538.

Wang, H. O., Tanaka, K. e Griffin, M. F. (2003). Fuzzy pole placement design with $H_{\infty}$ disturbance attenuation for uncertain nonlinear systems, IEEE Conference on Control Applications, Istanbul, Turquia, pp. 392-396. 